TRANSACTIONS OF THE

AMERICAN MATHEMATICAL SOCIETY

Volume 361, Number 10, October 2009, Pages 5223-5261

S 0002-9947(09)04633-9

Article electronically published on May 14, 2009

\title{
REDUCED STANDARD MODULES AND COHOMOLOGY
}

\author{
EDWARD T. CLINE, BRIAN J. PARSHALL, AND LEONARD L. SCOTT \\ We dedicate this paper to Toshiaki Shoji on the occasion of his 60th birthday
}

\begin{abstract}
First cohomology groups of finite groups with nontrivial irreducible coefficients have been useful in several geometric and arithmetic contexts, including Wiles's famous paper (1995). Internal to group theory, 1-cohomology plays a role in the general theory of maximal subgroups of finite groups, as developed by Aschbacher and Scott (1985). One can pass to the case where the group acts faithfully and the underlying module is absolutely irreducible. In this case, R. Guralnick (1986) conjectured that there is a universal constant bounding all of the dimensions of these cohomology groups. This paper provides the first general positive results on this conjecture, proving that the generic 1-cohomology $H_{\text {gen }}^{1}(G, L):=\lim _{q \rightarrow \infty} H^{1}(G(q), L)$ (see Cline, Parshall, Scott, and van der Kallen) (1977) of a finite group $G(q)$ of Lie type, with absolutely irreducible coefficients $L$ (in the defining characteristic of $G$ ), is bounded by a constant depending only on the root system. In all cases, we are able to improve this result to a bound on $H^{1}(G(q), L)$ itself, still depending only on the root system. The generic $H^{1}$ result, and related results for Ext ${ }^{1}$, emerge here as a consequence of a general study, of interest in its own right, of the homological properties of certain rational modules $\Delta^{\text {red }}(\lambda), \nabla_{\text {red }}(\lambda)$, indexed by dominant weights $\lambda$, for a reductive group $G$. The modules $\Delta^{\text {red }}(\lambda)$ and $\nabla_{\text {red }}(\lambda)$ arise naturally from irreducible representations of the quantum enveloping algebra $U_{\zeta}$ (of the same type as $G$ ) at a $p$ th root of unity, where $p>0$ is the characteristic of the defining field for $G$. Finally, we apply our Ext ${ }^{1}$-bounds, results of Bendel, Nakano, and Pillen (2006), as well as results of Sin (1993), (1992), (1994) on the Ree and Suzuki groups to obtain the (nongeneric) bounds on $H^{1}(G(q), L)$.
\end{abstract}

\section{INTRODUCTION}

Let $G$ be a semisimple, simply connected algebraic group defined over an algebraically closed field $k=\overline{\mathbb{F}}_{p}$. The group $G$ has a well-known construction, first discovered by Chevalley [11, in terms of the corresponding complex semisimple Lie algebra $\mathfrak{g}_{\mathbb{C}}$. In addition, given a dominant weight $\lambda$ for $\mathfrak{g}_{\mathbb{C}}$, one can construct two rational $G$-modules $\Delta(\lambda)$ and $\nabla(\lambda)$ by a process of "reduction modulo $p$ ". More precisely, $\Delta(\lambda)$ (resp., $\nabla(\lambda)$ ) is obtained from a minimal (resp., maximal)

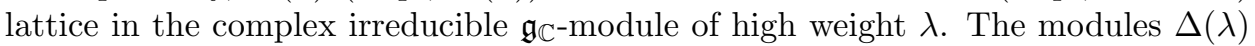
and $\nabla(\lambda)$ were broadly popularized by Steinberg [45] who also established some basic properties. In addition, these modules have remarkable homological properties, often dependent on the validity of Kempf's vanishing theorem (see, e.g.,

Received by the editors July 5, 2007 and, in revised form, September 5, 2007.

2000 Mathematics Subject Classification. Primary 20Gxx.

This research was supported in part by the National Science Foundation.

(c)2009 American Mathematical Society Reverts to public domain 28 years from publication 
[26. Prop. II.4.6]). A number of these properties were first discovered in [18. For example, if $\lambda, \mu$ are dominant weights, then $\operatorname{Ext}_{G}^{n}(\Delta(\lambda), \nabla(\mu))=0$ unless $n=0$ and $\lambda=\mu$, in which case the Ext group is a Hom and is equal to $k$. Thus, the modules $\Delta(\lambda)$ and $\nabla(\mu)$ not only have the same characters as in characteristic zero (where they identify with the irreducible module of high weight $\lambda$ ), but, suitably interpreted, these modules behave homologically the same way as in characteristic zero. (Also, they collectively form some kind of pair of dual bases at the derived category level.)

Let $U_{\zeta}$ be the (Lusztig) quantum enveloping algebra of the same type as $G$, where $\zeta$ is a $p$ th root of unity. The process described in the previous paragraph has been carried out by Lusztig [33] and then further studied by Lin 31, where now the role of $\mathfrak{g}_{\mathbb{C}}$ is played by $U_{\zeta}$. Thus, we obtain rational $G$-modules $\Delta^{\text {red }}(\lambda)$ and $\nabla_{\text {red }}(\lambda)$ which are indexed by $\lambda$. In many ways, these modules are quite analogous to the standard modules $\Delta(\lambda)$ and costandard modules $\nabla(\lambda)$, and they may be viewed as reduced versions of them. For example, $\Delta(\lambda)$ and $\Delta^{\text {red }}(\lambda)$ both have irreducible head $L(\lambda)$. The character of $\Delta(\lambda)$ is given by Weyl's character formula, while the character of $\Delta^{\text {red }}(\lambda)$ is given by the (Kazhdan-)Lusztig character formula (subject to some restrictions on $p$ ). In this paper, we begin an investigation into the homological properties of the $\Delta^{\text {red }}-$ and $\nabla_{\text {red }}$-modules, together with their deeper structural relationships to the $\Delta$ - and $\nabla$-modules.

To the category $G$-mod of finite-dimensional rational $G$-modules, we attach "enriched" Grothendieck groups $K_{0}^{L}$ and $K_{0}^{R}$. Both $K_{0}^{L}$ and $K_{0}^{R}$ are free modules over the ring $\mathbb{Z}\left[t, t^{-1}\right]$ of Laurent polynomials, and each has a basis indexed by the set $X^{+}$of dominant weights. These bases are paired to each other with respect to a natural sesquilinear pairing

$$
\langle,\rangle: K_{0}^{L} \times K_{0}^{R} \rightarrow \mathbb{Z}\left[t, t^{-1}\right] .
$$

The groups $K_{0}^{L}$ and $K_{0}^{R}$, first introduced in [13, can be viewed as deformations of the ordinary Grothendieck group $K_{0}$ of the bounded derived category $D^{b}(G-\bmod )$. However, $K_{0}$ is isomorphic to the ordinary Grothendieck group of $G$-mod, while $K_{0}^{L}$ and $K_{0}^{R}$ preserve homological degree information by taking into account parity conditions on modules. Given rational $G$-modules $M, N$, when $M$ and $N$ are "represented" in $K_{0}^{L}$ and $K_{0}^{R}$, respectively - say by $[M]$ and $[N]$ — then

$$
\sum_{i} \operatorname{dim} \operatorname{Ext}_{G}^{i}(M, N) t^{i}=\langle[M],[N]\rangle .
$$

Thus, if $[M],[N]$ can be explicitly written in terms of the paired bases alluded to above, (0.0.2) explicitly calculates $\operatorname{Ext}_{G}^{\bullet}(M, N)$ using the form (0.0.1).

More precisely, $M \in D^{b}\left(G\right.$-mod) is represented in $K_{0}^{L}$ when $M$ or its shift $M[1]$ belongs to $\mathcal{E}^{L}=\mathcal{E}^{L}(G-\bmod )$, and direct sums of such objects - those in $\mathcal{E}^{L} \oplus \mathcal{E}^{L}[1]$ are also represented in $K_{0}^{L}$. Here $\mathcal{E}^{L}$ is a full subcategory of $D^{b}(G-\bmod )$ roughly consisting of those objects which have a "filtration" with "sections" of the form $\Delta(\lambda)[i], \lambda \in X^{+}, i \equiv l(\lambda) \bmod 2$, for a suitable "length" function $l: X^{+} \rightarrow \mathbb{N}$. A similar description applies to $K_{0}^{R}$, involving another full subcategory $\mathcal{E}^{R}$ defined in terms of the modules $\nabla(\lambda)$. The "length" function $l$ could just as well be called a "parity" function, since we only use its values modulo 2. However, it often arises naturally in terms of lengths of Coxeter group elements associated with dominant weights. 
In a special situation, the question of when irreducible modules $L(\lambda)$ belong to $\widehat{\mathcal{E}^{L}}=\mathcal{E}^{L} \oplus \mathcal{E}^{L}[1]$ (and its counterpart $\widehat{\mathcal{E}^{R}}$ ) is closely related to the validity of certain character formulas. Specifically, let $X_{\mathrm{reg}}^{+} \subset X^{+}$be the set of regular dominant weights. Then a well-known theorem [13. Thm. 5.3] states that, given a saturated subset $\Gamma \subset X_{\mathrm{reg}}^{+}$(relative to the $\uparrow$ poset structure), $L(\lambda) \in \widehat{\mathcal{E} L}$ for all $\lambda \in \Gamma$ if and only if each $L(\lambda), \lambda \in \Gamma$, has formal character given by Lusztig's famous character formula.

The algebra $U_{\zeta}$ has a natural integral form $\widetilde{U}_{\zeta}$ over a discrete valuation ring with residue field $\mathbb{F}_{p}$. Let $\bar{U}_{\zeta}$ be the reduction modulo $p$ of $\widetilde{U}_{\zeta}$. As proved by Lusztig, the distribution (or hyper-)algebra hy $(G)$ of $G$ is a natural quotient of $k \otimes_{\mathbb{F}_{p}} \bar{U}_{\zeta}$. In this way, the irreducible (type 1 and integrable) $U_{\zeta}$-modules $L_{\zeta}(\lambda), \lambda \in X^{+}$, give rise by reduction modulo $p$ to certain rational $G$-modules $\Delta^{\text {red }}(\lambda)$ and $\nabla_{\text {red }}(\lambda)$. In the presence of Lusztig's (now largely known) conjecture for characters of irreducible representations of quantum groups at a root of unity, his modular conjecture may be viewed as stating the following: For $p \geq h$ (the Coxeter number of $G$ ) and $\lambda$ a regular dominant weight in the Jantzen region, $\Delta^{\text {red }}(\lambda) \cong \nabla_{\text {red }}(\lambda) \cong L(\lambda)$. An extension proposed by Kato posits the same result provided only that $p \geq h$ and $\lambda$ is a restricted dominant weight.

A main result, proved in Theorem6.8, establishes that, if the Kato version of the Lusztig conjecture holds, then $\Delta^{\text {red }}(\lambda) \in \widehat{\mathcal{E}^{L}}$ and $\nabla_{\text {red }}(\lambda) \in \widehat{\mathcal{E}^{R}}$ for all regular dominant weights $\lambda$. As a consequence, we are able to compute $\operatorname{Ext}_{G}^{\bullet}\left(\Delta^{\text {red }}(\lambda), \nabla_{\text {red }}(\mu)\right)$ for all regular weights $\lambda$ and $\mu$ in terms of Kazhdan-Lusztig polynomials; cf. Theorem 5.4(b). This result generalizes to all regular dominant weights a theorem of 13 for regular dominant weights inside the Jantzen region, where the modules $\Delta^{\text {red }}(\lambda), \nabla_{\text {red }}(\mu)$ are irreducible. In one substantive case when $\lambda=p \mu$ for a dominant weight $\mu$, we are even able to establish Theorem 5.4 without assuming the Lusztig conjecture. Section 6 contains additional results and conjectures concerning the modules $\Delta^{\text {red }}(\lambda)$ and $\nabla_{\text {red }}(\lambda)$. For example, we conjecture that, for $p>h$ and $\lambda$ a dominant weight, the standard module $\Delta(\lambda)$ has a filtration with sections of the form $\Delta^{\text {red }}(\mu)$ and, in Theorem 6.9, we prove a partial result in this direction.

In Section 7, we apply Theorem 6.8 and other arguments to show there is a bound on $\operatorname{dim} H^{1}(G, L)$, depending only on the root system and valid for any rational irreducible module $L$ and underlying characteristic $p$; cf. Theorem 7.3 . A similar, partial, result for $\operatorname{dim} \operatorname{Ext}_{G}^{1}\left(L, L^{\prime}\right)$ with both $L$ and $L^{\prime}$ irreducible is given in Theorem 7.7. (In the proofs of these results, the Lusztig conjecture is used, but only to handle very large primes, where the conjecture is a theorem; cf. 4.) Then we prove analogues of these results, Theorem 7.4 and Theorem 7.9, for generic cohomology $H_{\text {gen }}^{1}(G, L)$ (in the sense of [18) and its Ext ${ }^{1}$-analogue $\operatorname{Ext}_{G, \text { gen }}^{1}\left(L, L^{\prime}\right)$. Then Theorem 7.10 applies Theorem 7.7 on Ext ${ }_{G}^{1}$, together with some results of Bendel, Nakano, and Pillen [8], to bound $\operatorname{dim} H^{1}(G(q), L)$ in all cases for $G(q)=G_{\sigma}$, the fixed points of a surjective endomorphism $\sigma: G \rightarrow G$ with finite fixed points. Again, the bound depends only on the root system. (Results of P. Sin 42, 43], and [44] handle the Ree and Suzuki groups.) Theorems 7.4 and 7.10 provide the first general positive evidence for a conjecture given by R. Guralnick 21] that dimensions of 1-cohomology groups for finite groups $G_{0}$ with faithful, absolutely irreducible coefficients $L_{0}$ are universally bounded. As pointed out in 39] and as well as in the talk by Guralnick reported on in 25, this conjecture remains 
an open question. The current largest known dimension for $H^{1}\left(G_{0}, L_{0}\right)$ is 3 (see 39] and, more recently, [10]), but such cohomology groups are usually at most 1dimensional (another observation of Guralnick - see Theorem 7.11 for some related discussion). First cohomology groups of finite groups with irreducible coefficient modules are important for the general study of maximal subgroups of finite groups 6]. We believe our results here represent a significant step in understanding their asymptotic behavior, irrespective of the final verdict on Guralnick's conjecture. Some additional conjectures and questions for algebraic and quantum groups are given in the preambles to Lemma 7.6 and Remarks 7.8(a), (b) and (c). In addition, Remark 7.8(c) gives a detailed discussion of how the quantum approach of this section fits in with Andersen's 1984 work [1] on $\operatorname{Ext}_{G}^{1}\left(L, L^{\prime}\right)$.

Some early results in this paper were reported in 36. The second and third authors also thank the American Institute of Mathematics for financial support during the AIM workshop "Cohomology and representation theory for finite groups of Lie type: computational methods" held in June 2007. Participant comments during the meeting led the authors from Theorem 7.4 to Theorem 7.10. We would especially like to thank Bob Guralnick for asking leading questions, and we also thank Chris Bendel and Cornelius Pillen for providing some answers.

\section{Preliminaries}

After reviewing some notions from representation theory of semisimple groups and related quantum enveloping algebras, we introduce the modules $\Delta^{\text {red }}(\lambda)$ and $\nabla_{\text {red }}(\lambda)$.

1.1. General notation. Fix the algebraically closed field $k=\overline{\mathbb{F}}_{p}$ of positive characteristic $p$. If $H$ is a group scheme over $k$, let $H$-Mod (resp., $H$-mod) be the category of rational $H$-modules (resp., finite-dimensional rational $H$-modules). By definition, a rational $H$-module is a comodule for the coordinate algebra $k[H]$ of $H$; see [26, Ch. 2].

Let $G$ be a simply connected, semisimple algebraic group over $k$, defined and split over $\mathbb{F}_{p}$. There is a commutative Hopf algebra $A_{0}$ over $\mathbb{F}_{p}$ such that $k[G] \cong k \otimes_{\mathbb{F}_{p}} A_{0}$ as $k$-Hopf algebras. The Hopf algebra morphism $F^{*}: k[G] \rightarrow k[G]$ defined by putting $F^{*}(s \otimes a)=s \otimes a^{p}, s \in k, a \in A_{0}$, is the comorphism of the Frobenius morphism $F: G \rightarrow G$. If $V \in G$-Mod and $r$ is a positive integer, $V^{(r)}$ denotes the rational $G$-module obtained from $V$ by making $g \in G$ act on $V$ by $F^{r}(g)$. If $V \cong W^{(r)}$ for some rational $G$-module $W$, we write $W=V^{(-r)}$.

For a positive integer $r$, set $G_{r}=\operatorname{Ker} F^{r}$, the scheme-theoretic kernel of $F^{r}$. If $H$ is a subgroup scheme of $G$, then $G_{r} H=\left(F^{r}\right)^{-1}(H)$ denotes the pull-back through $F^{r}$ of $H$. In this paper, we consider only the case $r=1$ and the group schemes $G_{1}, G_{1} T$ and $G_{1} B$, where $T$ is a fixed maximal $\mathbb{F}_{p^{-}}$split torus and $B \supset T$ is a fixed Borel subgroup.

The Lie algebra $\mathfrak{g}$ of $G$ is a restricted Lie algebra; $u(\mathfrak{g})$ denotes its restricted enveloping algebra. Then $G_{1}$-mod (resp., $G_{1} T-\bmod , G_{1} B-\bmod$ ) is equivalent to $u(\mathfrak{g})-\bmod (\operatorname{resp} ., u(\mathfrak{g}) . T, u(\mathfrak{g}) . B$-mod). Here $u(\mathfrak{g})$-mod denotes the category of finite-dimensional $u(\mathfrak{g})$-modules, while $u(\mathfrak{g}) . T-\bmod$ and $u(\mathfrak{g}) . B-\bmod$ are the categories of finite-dimensional $u(\mathfrak{g})$-modules with a compatible (rational) action of $T$ and $B$, respectively.

Let $X=X(T)$ be the character group and $X^{\vee}=\operatorname{Hom}\left(\mathbb{G}_{m}, T\right)$ the cocharacter group of $T$. There is a natural pairing $():, X \times X^{\vee} \rightarrow \mathbb{Z} \cong \operatorname{End}\left(\mathbb{G}_{m}\right)$. Let 
$\Phi \subset X(T)$ be the root system of $G$ with respect to $T$. If $\alpha \in \Phi$, let $\alpha^{\vee} \in X^{\vee}$ be the associated coroot. Let $Q:=\mathbb{Z} \Phi \subset X(T)$ be the root lattice.

Let $\Phi^{+}$be a fixed set of positive roots, and let $\Pi=\left\{\alpha_{1}, \cdots, \alpha_{n}\right\} \subseteq \Phi^{+}$be the simple roots in $\Phi$. We assume that if $B^{+} \supseteq T$ is the positive Borel subgroup defined by $\Phi^{+}$, then $B$ (the original Borel subgroup) is its opposite Borel subgroup (and so corresponds to the set $\Phi^{-}:=-\Phi^{+}$of negative roots). We regard $X$ as a poset by putting $\lambda \leq \mu$, for $\lambda, \mu \in X$, if and only if $\mu-\lambda \in \mathbb{N} \Phi^{+}$. A second partial order $\uparrow$ on $X$ is defined in terms of the affine Weyl group discussed below.

Let $X^{+} \subset X$ be the set of dominant weights on $T$, i.e., $\lambda \in X$ belongs to $X^{+}$if and only if $\left(\lambda, \alpha^{\vee}\right) \in \mathbb{Z}^{+}$for all $\alpha \in \Pi$. Denote the fundamental dominant weights by $\varpi_{1}, \cdots, \varpi_{n}$; thus, $\left(\varpi_{i}, \alpha_{j}^{\vee}\right)=\delta_{i, j}$. We list $\Pi$ and the fundamental dominant weights as in [9, Appendix]. For $r \geq 1$, let $X_{r}^{+}$be the set of $r$-restricted dominant weights, i.e., $\lambda \in X_{1}^{+}$if and only if $0 \leq\left(\lambda, \alpha^{\vee}\right)<p^{r}$ for all $\alpha \in \Pi$. (When $r=1$, we refer to elements of $X_{1}^{+}$simply as restricted dominant weights.)

1.2. The affine and extended affine Weyl groups. Let $\mathbb{E}=\mathbb{R} \otimes_{\mathbb{Z}} X$ be endowed with a positive definite, symmetric bilinear form (, ), invariant under the Weyl group $W$ of $\Phi$. We identify $X^{\vee}$ as a subgroup of $\mathbb{E}$, so that $\alpha^{\vee}=\frac{2}{(\alpha, \alpha)} \alpha$ and the pairing $X \times X^{\vee} \rightarrow \mathbb{Z}$ is compatible with the inner product.

The affine Weyl group $W_{p}=p \mathbb{Z} \Phi \rtimes W$ is the group of transformations on $\mathbb{E}$ generated by $W$ and the normal subgroup consisting of translations by elements in $p \mathbb{Z} \Phi$. If $\alpha \in \Phi$ and $r \in \mathbb{Z}$, define $s_{\alpha, r}: \mathbb{E} \rightarrow \mathbb{E}$ by $s_{\alpha, r}(x)=x-\left(\left(x, \alpha^{\vee}\right)-r p\right) \alpha$. Then $s_{\alpha, r} \in W_{p}$. In general, $W_{p}$ is a Coxeter group. If $\Phi$ is irreducible and if $\alpha_{0} \in \Phi$ denotes the maximal short root, then $\left(W_{p}, S_{p}\right)$ is a Coxeter system, with $S_{p}=\left\{s_{\alpha_{1}}, \cdots, s_{\alpha_{n}}, s_{\alpha_{0},-1}\right\}$. Considerations in this paper often reduce to the irreducible (single component) case.

In this paper, we will usually use the "dot" action of $W_{p}$ on $\mathbb{E}$, given by setting $w \cdot x=w(x+\rho)-\rho$, where $\rho=\frac{1}{2} \sum_{\alpha \in \Phi^{+}} \alpha$ is the Weyl weight. Let $C^{+} \subset \mathbb{E}$ be the positive fundamental alcove; it consists of all $x \in \mathbb{E}$ satisfying, component by component, the inequalities $0<\left(x+\rho, \alpha_{i}^{\vee}\right), i=1, \cdots, n$, and $\left(x+\rho, \alpha_{0}^{\vee}\right)<p$. Put $C^{-}=w_{0} \cdot C^{+}$, where $w_{0}$ is the maximal word in $W$. The closures $\overline{C^{+}}$and $\overline{C^{-}}$are fundamental domains for the action of $W_{p}$ on $\mathbb{E}$. The subsets $w \cdot C^{+} \subset \mathbb{E}, w \in W_{p}$, are the alcoves for $W_{p}$. If $C=w \cdot C^{+}$is an alcove, put $C_{\mathbb{Z}}=C \cap X$ and $\bar{C}_{\mathbb{Z}}=\bar{C} \cap X$.

Using $W_{p}$ we can define the $\uparrow$ partial ordering on $X$ as follows: For $\lambda, \mu \in X$, $\lambda \uparrow \mu$ if and only if there is a sequence $\lambda=\lambda_{0} \leq \lambda_{1} \leq \cdots \leq \lambda_{t}=\mu$ in which, for $0 \leq i<t, \lambda_{i+1}=s_{\beta_{i}, n_{i}} \cdot \lambda_{i}$ for some $\beta_{i} \in \Phi$ and some $n_{i} \in \mathbb{Z}$. (This partial ordering, taken from [26, 6.4], differs somewhat from that defined in [13, p. 527], although the two agree on $X^{+}-\rho$.)

When $\Phi$ is irreducible, the walls of the simplex $\overline{C^{-}}$are labeled by the simple reflections $s_{\alpha_{0},-1}, s_{\alpha_{1}}, \cdots, s_{\alpha_{n}}$. Thus, the walls of any alcove $C$ are labeled by the same set of simple reflections, since there is a unique $w \in W_{p}$ satisfying $w \cdot C=C^{-}$. Given $s \in S_{p}$, we can speak of the $s$-wall of an alcove $C$. Thus, the dot action of $W_{p}$ carries an $s$-wall into an $s$-wall. If $\lambda \in C$, let $C s$ be the alcove which is obtained from $C$ by reflection through the $s$-wall of $C$. Similarly, given $\lambda \in C$ and $s \in S_{p}$, let $\lambda s \in C s$ be the image of $\lambda$ through reflection through the $s$-wall of $C$. Similar remarks hold when $\Phi$ is not irreducible.

The extended affine Weyl group $\widetilde{W}_{p}$ is defined to be $\widetilde{W}_{p}=p X \rtimes W$. Clearly, $W_{p} \unlhd \widetilde{W}_{p}$ and $\widetilde{W}_{p} / W_{p} \cong X / Q$ is a finite abelian group. Also, $\widetilde{W}_{p}$ acts faithfully 
on $\mathbb{E}$, preserving the alcoves. For $\xi \in X$, it is convenient to denote by $t_{p \xi}$ the corresponding element in $\widetilde{W}_{p}$. As an operator on $\mathbb{E}$, it is translation by $p \xi$. Define $N$ to be the stabilizer in $\widetilde{W}_{p}$ of $C^{+}$. Then $\widetilde{W}_{p}=N \rtimes W_{p}$ and $N \cong X / Q$.

Let $\phi: \widetilde{W}_{p} \rightarrow W$ be the surjective homomorphism with kernel $p X$. For $\alpha \in \Phi$, $r \in \mathbb{Z}$, and $w \in \widetilde{W}_{p}, w s_{\alpha, r} w^{-1}=s_{\phi(w) \alpha, r+\left(\xi, \phi(w)(\alpha)^{\vee}\right)}$ if $w=t_{p \xi} \phi(w), \xi \in X$. Thus, $\widetilde{W}_{p}$ acts on the set of reflections $s_{\alpha, r}$ in $\widetilde{W}_{p}$, and $N$ is also isomorphic to the stabilizer in $\widetilde{W}_{p}$ of $S_{p}$.

The length function $l: W_{p} \rightarrow \mathbb{N}$ for the Coxeter system $\left(W_{p}, S_{p}\right)$ extends to a length function $l: \widetilde{W}_{p} \rightarrow \mathbb{N}$, setting $l(n w)=l(w)$, for $n w \in \widetilde{W}_{p}, n \in N, w \in W_{p}$. By the previous paragraph, $l(w n)=l(w)$. The length function defines a function $l: X \rightarrow \mathbb{N}$ as follows. Given $\lambda \in X$, choose $w \in W_{p}$ of minimal length such that $w \cdot \lambda \in \overline{C^{-}}$. Put $l(\lambda):=l(w)$.

A weight $\lambda \in X$ is regular provided that $\lambda \in C$ for some alcove $C$. Let $X_{\text {reg }}$ be the set of regular weights, so that $X_{\text {reg }} \neq \emptyset$ if and only if $p \geq h$. Otherwise, $\lambda$ is called singular. We let $X_{\text {reg }}^{+}$be the set of all regular dominant weights.

1.3. Representation theory. Given a closed subgroup scheme $H$ of a group scheme $K$, the restriction functor $\operatorname{res}_{H}^{K}: K$-Mod $\rightarrow H$-Mod has a left exact right adjoint $\operatorname{ind}_{H}^{K}: H$-Mod $\rightarrow K$-Mod (the induction functor). For a nonnegative integer $n$, let $R^{n} \operatorname{ind}_{H}^{K}: K-\operatorname{Mod} \rightarrow H-\operatorname{Mod}$ be the $n$th right derived functor of $\operatorname{ind}_{H}^{K}$.

We first consider the representation theory of the semisimple group $G$. For $\lambda \in X^{+}$, let $L(\lambda) \in G$-mod be the irreducible representation of high weight $\lambda$. Write $\nabla(\lambda)=\operatorname{ind}_{B}^{G} \lambda$, where $\lambda$ here denotes the one-dimensional rational $B$-module defined by extending $\lambda$ to a linear character on $B$ through the quotient map $B \rightarrow$ $B / U \cong T$. If $w_{0} \in W$ is the long word, define $\star: X \rightarrow X$ by $\tau \mapsto \tau^{\star}:=-w_{0}(\tau)$. Set $\Delta(\lambda)=\nabla\left(\lambda^{\star}\right)^{*}$, where $(-)^{*}$ means linear dual. The module $\nabla(\lambda)$ (resp., $\left.\Delta(\lambda)\right)$ has socle (resp., head) isomorphic to $L(\lambda)$. We call $\Delta(\lambda)$ (resp., $\nabla(\lambda))$ the standard (resp., costandard) $G$-module of high weight $\lambda$. The $\Delta(\lambda)$ (resp., $\nabla(\lambda)), \lambda \in X^{+}$, form the standard (resp., costandard) modules in the highest weight categories $G$-mod and $G$-Mod.

The integral group ring $\mathbb{Z} X$ of $X$ has $\mathbb{Z}$-basis $e^{\xi}, \xi \in X$. If $V \in T$-mod, ch $V \in$ $\mathbb{Z} X$ denotes the formal character of $V$. If $\chi=\sum n_{\xi} e^{\xi} \in \mathbb{Z} X$, set $\chi^{(1)}=\sum n_{\xi} e^{p \xi}$. Thus, ch $V^{(1)}=(\operatorname{ch} V)^{(1)}$. For $\lambda \in X^{+}$, ch $\Delta(\lambda)=\operatorname{ch} \nabla(\lambda)=\chi(\lambda)$, where

$$
\chi(\lambda):=\sum_{w \in W}(-1)^{l(w)} e^{w \cdot \lambda} / \sum_{w \in W}(-1)^{l(w)} e^{w \cdot 0} \text { (Weyl character formula). }
$$

Given $M \in G-\bmod$, its Poincaré polynomial is

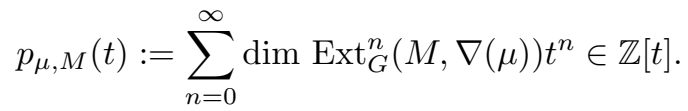

For later use, we recall the following well-known cohomological fact. For (a), see [13, Lemma 2.2], and for part (b), see [13, Lemma 3.2]. However, (b) holds by (a) if $M$ has a filtration by $G$-modules with $\Delta$-sections (i.e., sections of the form $\Delta(\lambda)$, $\left.\lambda \in X^{+}\right)$. In particular, it holds if $M \cong L(\lambda)$ with $\lambda$ minimal. Next, the function $p_{\mu, M}(-1)$ is additive on short exact sequences in $G$-mod. Then (b) follows easily for simple modules $L(\lambda)$ by induction on $\lambda$, and thus holds generally. 
Lemma 1.1. (a) For $\lambda, \mu \in X^{+}, \operatorname{dim} \operatorname{Ext}_{G}^{n}(\Delta(\lambda), \nabla(\mu))=\delta_{n, 0} \delta_{\lambda, \mu}$.

(b) For any finite-dimensional rational $G$-module $M$,

$$
\operatorname{ch} M=\sum_{\mu \in X^{+}} p_{\mu, M}(-1) \chi(\mu) .
$$

If $V \in B$-mod, let $\mathcal{L}_{V}$ be the induced $G$-homogenous vector bundle on $G / B$ with fibre $V$ at $B / B$. Write $H^{\bullet}(G / B, V)$ for $H^{\bullet}\left(G / B, \mathcal{L}_{V}\right)$. Thus, for $\lambda \in X^{+}$, $\nabla(\lambda) \cong H^{0}(G / B, \lambda)$ and $\Delta(\lambda) \cong H^{N}\left(G / B, w_{0} \cdot \lambda\right)$, where $N=\operatorname{dim} G / B$.

Given $V \in B$-mod, its Euler characteristic is

$$
\chi(V):=\sum_{n=0}^{\infty}(-1)^{n} \operatorname{ch} H^{n}(G / B, V) \in \mathbb{Z} X
$$

Suppose that $\gamma \in X$. If there exists $x \in W$ such that $x \cdot \gamma=\gamma^{+} \in X^{+}$, we say that $\gamma$ is $W$-regular. In this case, the Weyl group element $x$ is uniquely determined. Otherwise, we say that $\gamma$ is $W$-singular. Letting $V=\chi$, we have

$$
\chi(\gamma)=\left\{\begin{array}{l}
(-1)^{l(x)} \chi\left(\gamma^{+}\right)=(-1)^{l(x)} \operatorname{ch} \Delta\left(\gamma^{+}\right), \quad \gamma \text { is } W \text {-regular } \\
0, \quad \text { otherwise. }
\end{array}\right.
$$

When $\gamma=\gamma^{+} \in X^{+},(1.1 .3)$ is consistent with (1.0.3).

We require some facts about the representation theory of the group schemes $G_{1} B$ and $B_{1} T$. The set $X$ indexes the irreducible objects in both $G_{1} B-\bmod$ and $G_{1} T$-mod. Thus, if $\lambda \in X$, let $\widehat{L}_{1}(\lambda)$ be the irreducible $G_{1} B$-module of high weight $\lambda$. Its restriction to $G_{1} T$, which we usually continue to denote by $\widehat{L}_{1}(\lambda)$, remains irreducible. Given $\lambda \in X$, write $\lambda=\lambda_{0}+p \lambda_{1}$, where $\lambda_{0} \in X_{1}^{+}$and $\lambda \in X$. Then in $G_{1} B-\bmod$ or $G_{1} T-\bmod , \widehat{L}_{1}(\lambda) \cong p \lambda_{1} \otimes \widehat{L}_{1}\left(\lambda_{0}\right)$, where $p \lambda_{1}$ denotes the one-dimensional module defined by the character $p \lambda_{1}$ on $G_{1} B$. In addition, $\widehat{L}_{1}\left(\lambda_{0}\right) \cong \operatorname{res}_{G_{1} B}^{G} L\left(\lambda_{0}\right)$.

Lemma 1.2. For any rational $G_{1} B$-module $V$, we have

$$
R^{n} \operatorname{ind}_{G_{1} B}^{G} V^{(1)} \cong\left(R^{n} \operatorname{ind}_{B}^{G} V\right)^{(1)}
$$

for any non-negative integer $n$.

Proof. In degree 0,

$$
\begin{aligned}
\operatorname{ind}_{G_{1} B}^{G} V^{(1)} & \cong\left(k[G] \otimes V^{(1)}\right)^{G_{1} B} \\
& \cong\left(k[G]^{G_{1}} \otimes V^{(1)}\right)^{B} \\
& \cong\left(k\left[G / G_{1}\right] \otimes V\right)^{B / B_{1}} \\
& \cong\left(\operatorname{ind}_{B}^{G} V\right)^{(1)},
\end{aligned}
$$

functorially in $V$. Let $I \in G_{1} B$-mod be injective. Then $I$ is also an injective object in $B$-mod, so that the required isomorphism holds in all degrees by dimension shifting.

Given $\lambda \in X$, put $\widehat{Z}_{1}(\lambda):=u(\mathfrak{g}) \otimes_{u\left(\mathfrak{b}^{+}\right)} k_{\lambda}$. Then $\widehat{Z}_{1}(\lambda) \in G_{1} B-\bmod$ has dimension $p^{N}$, where $N=\left|\Phi^{+}\right|$, and has irreducible head $\widehat{L}_{1}(\lambda)$. 
1.4. Character formulas. Let $\lambda \in X^{+}$and write $\lambda=w \cdot \lambda^{-}$, where $\lambda^{-} \in \overline{C_{\mathbb{Z}}^{-}}$ and $w$ has minimal length among all elements $w^{\prime} \in W_{p}$ which satisfy $w^{\prime} \cdot \lambda^{-}=\lambda$. Because the isotropy subgroup of $\lambda^{-}$in $W_{p}$ has the form $W_{J}$ for some $J \subset S_{p}, w$ is uniquely determined as a distinguished left coset representative of $W_{J}$ in $W$. For $y, w \in W_{p}$, let $P_{y, w} \in \mathbb{Z}[t]$ be the associated Kazhdan-Lusztig polynomial 1$]$ Define

$$
\chi_{\mathrm{KL}}(\lambda)=\sum_{y \in W_{p}, y \cdot \lambda^{-} \in X^{+}}(-1)^{l(w)-l(y)} P_{y, w}(-1) \chi\left(y \cdot \lambda^{-}\right) .
$$

The following result is proved by Kato for $p \geq h$, but the argument works for all $p$. (In Kato's argument, replace the weight $\lambda$ in the interior of an alcove by a weight in its closure.)

Lemma 1.3 (Kato [28]). Let $\lambda \in X^{+}$have expansion $\lambda=\lambda_{0}+p \lambda_{1}$, where $\lambda_{0} \in X_{1}^{+}$ and $\lambda_{1} \in X^{+}$. Then

$$
\chi_{\mathrm{KL}}(\lambda)=\chi_{\mathrm{KL}}\left(\lambda_{0}\right) \chi\left(\lambda_{1}\right)^{(1)} .
$$

Following [35, we say that $\lambda \in X^{+}$satisfies the Lusztig character formula (LCF) provided that $\operatorname{ch} L(\lambda)=\chi_{\mathrm{KL}}(\lambda)$. Also, we say that $\lambda=w \cdot \lambda^{-}$satisfies the homological LCF $(\mathrm{hLCF})$ provided that

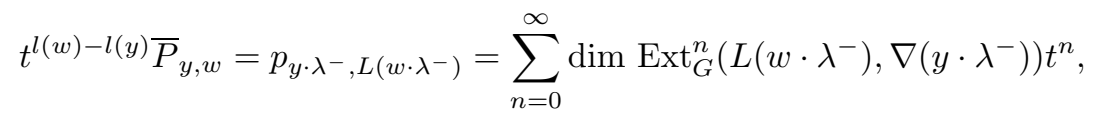

whenever $y \cdot \lambda^{-} \in X^{+}$. In this expression, $\bar{P}_{y, w}$ is obtained from $P_{y, w}$ by replacing $t$ by $t^{-1}$ throughout, and the second equality is just the definition (1.0.4) of the

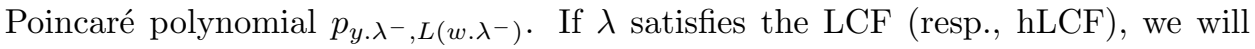
often say that $L(\lambda)$ satisfies the LCF (resp., hLCF) condition. Actually, using (1.3.1), it makes sense, for any module $M(\lambda)$, labeled by $\lambda=w \cdot \lambda^{-}$to say $M(\lambda)$ satisfies the hLCF condition.

Although the definitions in the previous paragraph have been made for arbitrary $p$, we will be concerned with the special case when $p \geq h$ (so that $X_{\text {reg }}^{+} \neq \emptyset$ ).

1.5. Quantum enveloping algebras. When discussing the quantum enveloping algebra $U_{\zeta}$ below associated to $G, \zeta$ will be a $p$ th root of unity. In order to apply the results of 5 , we will always assume that the prime $p$ is odd, and, if $G$ has a component of type $G_{2}$, then $p>3$. Let $C=\left(c_{i, j}\right)$ be the Cartan matrix of $G$ : thus, $c_{i, j}=\left(\alpha_{j}, \alpha_{i}^{\vee}\right)$, for $1 \leq i, j \leq n$.

Inside the function field $\mathbb{Q}(v)$, let $\mathcal{A}=\mathbb{Z}[v]_{\mathfrak{m}}$, where $\mathfrak{m}=(v-1, p)$. The quantum enveloping algebra $U^{\prime}$ over $\mathbb{Q}(v)$ corresponding to the matrix $C$ has generators $E_{1}, \cdots, E_{n}, F_{1}, \cdots, F_{n}, K_{1}^{ \pm 1}, \cdots, K_{n}^{ \pm 1}$ satisfying the familiar relations; cf. 27. Let $U$ be the $\mathcal{A}$-subalgebra of $U^{\prime}$ generated by the divided powers $E_{i}^{(N)}, F_{i}^{(N)}$, $N \geq 1$, together with the elements $K_{i}^{ \pm 1}, 1 \leq i \leq n$.

\footnotetext{
${ }^{1}$ Recall that $P_{y, w}$ is a polynomial in $q:=t^{2}$. We prefer to regard $P_{y, w}$ as a polynomial in $t$, albeit one which is also a polynomial in $t^{2}$. Unless $y \leq w, P_{y, w}=0$. If $y=w$, then $P_{y, w}=1$. If $y<w, P_{y, w}$ has degree (in $\left.t\right) \leq \ell(w)-\ell(y)-1$. If $y<w$, let $\mu(y, w)$ be the coefficient of $t^{\ell(w)-\ell(y)-1}$; otherwise, put $\mu(y, w)=0$.

${ }^{2}$ Let $F$ be the unique facet containing $\lambda$. Then, using [26 6.11], $F$ lies in the upper closure of a unique alcove $C$. If $C^{\prime}$ is a second alcove satisfying $F \subseteq \overline{C^{\prime}}$, then $C \uparrow C^{\prime}$. If $w \in W_{p}$ satisfies $w \cdot C^{-}=C$, then $w$ is the shortest element in $W_{p}$ satisfying $w \cdot \lambda^{-}=\lambda$. In the expression below, given $\mu \in X^{+}$, there may well exist several $y \leq w$ such that $y \cdot \lambda^{-}=\mu$.
} 
Let $\mathcal{O}=\mathcal{A} /\left(\phi_{p}\right)$, where $\phi_{p}=1+v+\cdots+v^{p-1} \in \mathfrak{m}$ is the $p$ th cyclotomic polynomial. Thus, $\mathcal{O}$ is a discrete valuation ring with maximal ideal $\mathfrak{n}=(p)$, quotient field denoted $K$ and residue field $\mathbb{F}_{p}$. Then $\zeta:=v+\left(\phi_{p}\right) \in \mathcal{O}$ is a primitive $p$ th root of unity. Regarding $\mathcal{O}$ as an $\mathcal{A}$-module via $v \mapsto \zeta, \widetilde{U}_{\zeta}:=(\mathcal{O} \otimes U)$. Set $U_{\zeta}=K \otimes U$. Thus, $\widetilde{U}_{\zeta}$ is an integral $\mathcal{O}$-form of $U_{\zeta}$. Put $\bar{U}_{\zeta}=\widetilde{U}_{\zeta} / \pi \widetilde{U}_{\zeta}$, and let $I$ be the ideal in $\bar{U}_{\zeta}$ generated by the images of the elements $K_{i}-1,1 \leq i \leq n$. By [33. (8.15)],

$$
\bar{U}_{\zeta} / I \cong \operatorname{hy}_{0}(G)
$$

the distribution algebra of $G$ over $\mathbb{F}_{p}$. Put hy $(G)=k \otimes_{\mathbb{F}_{p}}$ hy $_{0}(G)$.

Remark 1.4. In practice, it will be more convenient to enlarge $\mathcal{O}$ to be a principal ideal domain with residue field the algebraically closed field $k$. In what follows, we will still let $\mathcal{O}$ denote this PID and let $\mathfrak{m}=(\pi)$ be its maximal ideal. Continue to let $K$ be the quotient field of $\mathcal{O}$.

The category of finite-dimensional integrable, type $1 U_{\zeta}$-modules will be denoted by $\mathcal{C}_{\zeta}$. It is a highest weight category with irreducible (resp. standard, costandard) modules $L_{\zeta}(\lambda)$ (resp., $\left.\Delta_{\zeta}(\lambda), \nabla_{\zeta}(\lambda)\right), \lambda \in X^{+}$. For $\mu \in X^{+}, \operatorname{ch} \Delta_{\zeta}(\mu)=\operatorname{ch} \nabla_{\zeta}(\mu)=$ $\chi(\mu)$. In particular, the quantum version of Lemma 1.1 holds.

Remark 1.5. As noted above, the irreducible, type 1, integrable $U_{\zeta}$ modules are indexed by $X^{+}$. Subject to some possible restrictions on $p$, for any $\lambda \in X^{+}$, $\operatorname{ch} L_{\zeta}(\lambda)=\chi_{\mathrm{KL}}(\lambda)$. In these cases, we say $\lambda$ satisfies the $\mathrm{LCF}_{\zeta}$ condition. In type $A_{n}$ and $D_{2 n}$, there is no restriction on $p$ (except our blanket assumption that $p$ is odd). In type $D_{2 n+1}$, we require $p>3$. In other types, we require $p>h$; see [46, §7] for a detailed discussion and further references. Throughout this paper, unless otherwise explicitly noted, we will always assume that the $L C F_{\zeta}$ holds for all $\lambda \in X^{+}$. In addition, for $\lambda=w \cdot \lambda^{-} \in X_{\text {reg }}^{+}$with $\lambda^{-} \in C_{\mathbb{Z}}^{-}$, we have

$$
t^{l(w)-l(y)} \bar{P}_{y, w}=\sum_{n=0}^{\infty} \operatorname{dim} \operatorname{Ext}_{\mathcal{C}_{\zeta}}^{n}\left(L_{\zeta}\left(w \cdot \lambda^{-}\right), \nabla_{\zeta}\left(y \cdot \lambda^{-}\right)\right) t^{n} .
$$

In other words, the $\mathrm{hLCF}_{\zeta}$ holds for $\lambda$. It is unknown to the authors if the assumption that $\lambda$ is regular in (1.5.1) is necessary. In addition, we have, given $x \cdot \lambda^{-}, y \cdot \lambda^{-} \in X_{\mathrm{reg}}^{+}$,

$$
\sum_{n=0}^{\infty} \operatorname{dim} \operatorname{Ext}_{\mathcal{C}_{\zeta}}^{n}\left(L_{\zeta}\left(x \lambda^{-}\right), L_{\zeta}\left(y \cdot \lambda^{-}\right)\right) t^{n}=\sum_{z} t^{l(x)+l(y)-2 l(z)} \bar{P}_{z, x} \bar{P}_{z, y},
$$

where the summation on the right is over all $z \in W_{p}$ for which $z \cdot(-2 \rho) \in X^{+}$. See the argument in [13, Thm. 3.9.1]. A special case of (1.5.2) will be important later. If $y<x$ with $y \cdot \lambda^{-}, w \cdot \lambda^{-} \in X_{\text {reg, }}^{+}$, we have

$$
\operatorname{dim} \operatorname{Ext}_{\mathcal{C}_{\zeta}}^{1}\left(L_{\zeta}\left(x \cdot \lambda^{-}\right), L_{\zeta}\left(y \cdot \lambda^{-}\right)\right)=\operatorname{dim}_{\operatorname{Ext}_{\mathcal{C}_{\zeta}}^{1}}\left(L_{\zeta}\left(y \cdot \lambda^{-}\right), L_{\zeta}\left(x \cdot \lambda^{-}\right)\right)=\mu(y, x) .
$$

(See footnote 1 for notation.)

An admissible lattice in $M \in \mathcal{C}_{\zeta}$ is a free $\mathcal{O}_{\text {-submodule }} \widetilde{M}$ of $M$ such that $\widetilde{M}_{K}=K \otimes_{\mathcal{O}} \widetilde{M} \cong M$ and such that $\widetilde{M}$ is $\widetilde{U}_{\zeta}$-stable. If $M_{\mu}$ is a nonzero weight space in $M$, then $\widetilde{M} \cap M_{\mu}$ contains a basis for $M_{\mu}$ [20, (3.3)]. Define $\widetilde{\mathcal{C}_{\zeta}}$ to be the 
category of integrable, type $1 \widetilde{U}_{\zeta}$-modules which are finitely generated over $\mathcal{O}$; see 20] for a discussion. For $M, N \in \mathcal{C}_{\zeta}$, we have

$$
\operatorname{Ext}_{\tilde{\mathcal{C}}_{\zeta}}^{\bullet}(\widetilde{M}, \widetilde{N})_{K} \cong \operatorname{Ext}_{\mathcal{C}_{\zeta}}^{\bullet}(M, N)
$$

whenever $\widetilde{M}, \widetilde{N}$ are admissible lattices for $M, N$, respectively. In addition, suppose that $\widetilde{M}$ is an admissible lattice as above, and put $\bar{M}:=\widetilde{M} / \pi \widetilde{M}$. For any $V \in$ $G-\bmod$,

$$
\operatorname{Ext}_{\widetilde{\mathcal{C}}_{\zeta}}^{\bullet}(\widetilde{M}, V) \cong \operatorname{Ext}_{G}^{\bullet}(\bar{M}, V) .
$$

The proof of (1.5.5) is given in [20, (2.9) \& Thm. 3.2]. The proof of [20, (2.9)] (a reduction to standard issues with projective resolutions) also establishes (1.5.4).

In addition, for $\lambda \in X^{+}$, there exist admissible lattices $\widetilde{\Delta}_{\zeta}(\lambda)$ and $\widetilde{\nabla}_{\zeta}(\lambda)$ for $\Delta_{\zeta}(\lambda)$ and $\nabla_{\zeta}(\lambda)$, respectively, so that $\widetilde{\Delta}_{\zeta}(\lambda) / \pi \widetilde{\Delta}_{\zeta}(\lambda) \cong \Delta(\lambda)$ and $\widetilde{\nabla}_{\zeta}(\lambda) / \pi \widetilde{\nabla}_{\zeta}(\lambda) \cong$ $\nabla(\lambda)$ [20, pp. 149,158-159]. The lattice $\widetilde{\Delta}_{\zeta}(\lambda)$ is generated as a $\widetilde{U}_{\zeta}$-module by a high weight vector in $\Delta_{\zeta}(\lambda)$.

Given an irreducible $U_{\zeta}$-module $L_{\zeta}(\lambda)$ of high weight $\lambda$, fix a high weight vector $v^{+} \in L_{\zeta}(\lambda)$. Then there is a unique admissible lattice $\widetilde{L}_{\zeta}^{\min }(\lambda)\left(\operatorname{resp} ., \widetilde{L}_{\zeta}^{\max }(\lambda)\right)$ of $L_{\zeta}(\lambda)$ which is minimal (resp., maximal) with respect to all admissible lattices $\widetilde{L}$ such that $\widetilde{L} \cap L_{\zeta}(\lambda)_{\lambda}=\mathcal{O} v^{+}$. For example, we can take $\widetilde{L}_{\zeta}^{\min }(\lambda)=\widetilde{U}_{\zeta} \cdot v^{+}$. By abuse of notation, we call $\widetilde{L}_{\zeta}^{\min }(\lambda)$ (resp., $\widetilde{L}_{\zeta}^{\max }(\lambda)$ ) the minimal (resp., maximal) lattice of $L_{\zeta}(\lambda)$. Any two "minimal" (resp., "maximal") lattices are isomorphic as $\widetilde{U}_{\zeta \text {-modules. }}$

For $\lambda \in X^{+}$, put

$$
\Delta^{\mathrm{red}}(\lambda)=\widetilde{L}_{\zeta}^{\min }(\lambda) / \pi \widetilde{L}_{\zeta}^{\min }(\lambda) \text { and } \nabla_{\mathrm{red}}(\lambda)=\widetilde{L}_{\zeta}^{\max }(\lambda) / \pi \widetilde{L}_{\zeta}^{\max }(\lambda)
$$

The rational $G$-module $\Delta^{\text {red }}(\lambda)$ (resp., $\nabla_{\text {red }}(\lambda)$ ) is the reduced standard (resp., costandard) module of high weight $\lambda$. Under the assumption that the LCF holds for $U_{\zeta}$ for all $\lambda \in X^{+}$(see Remark 1.5),

$$
\operatorname{ch} \Delta^{\mathrm{red}}(\lambda)=\operatorname{ch} \nabla_{\mathrm{red}}(\lambda)=\chi_{\mathrm{KL}}(\lambda)
$$

for all $\lambda \in X^{+}$.

The natural surjection $\Delta_{\zeta}(\lambda) \rightarrow L_{\zeta}(\lambda)$ restricts, by the discussion above, to a surjection $\widetilde{\Delta}_{\zeta}(\lambda) \rightarrow L_{\zeta}^{\min }(\lambda)$. After passage to the field $k$, we obtain a surjection $\Delta(\lambda) \rightarrow \Delta^{\text {red }}(\lambda)$. Thus, $\Delta^{\text {red }}(\lambda)$ has irreducible head $L(\lambda)$. Dually, there is an inclusion $\nabla_{\text {red }}(\lambda) \hookrightarrow \nabla(\lambda)$, so that $\nabla_{\text {red }}(\lambda)$ has irreducible socle $L(\lambda)$.

In order to give another description of $\Delta^{\text {red }}(\lambda)$, we need an unpublished result of Cline.

Lemma 1.6 (E. Cline). For a restricted weight $\lambda \in X_{1}^{+}$, there is a surjective morphism $\widehat{Z}_{1}(\lambda) \rightarrow \Delta(\lambda)$ in $G_{1} B-\bmod$.

Proof. Let $v^{+} \in \Delta(\lambda)$ be a high weight vector. There is a natural $G_{1} B$-module map $\widehat{Z}_{1}(\lambda) \rightarrow \Delta(\lambda)$ which sends $u \otimes 1 \in \widehat{Z}_{1}(\lambda)$ to $u \cdot v^{+}, u \in \mathfrak{g}$. The image $E:=u(\mathfrak{g}) \cdot v^{+}=u(\mathfrak{b}) \cdot v$ of this map is a $u(\mathfrak{g})$-submodule of $\Delta(\lambda)$. Of course, $v^{+} \in E$, and we must show that $\Delta(\lambda)=k G \cdot v^{+} \subseteq E$.

Let $\alpha \in-\Pi$ be the negative of a simple root and fix an $\alpha$-root vector $x_{\alpha} \in \mathfrak{g}$. For a positive integer $n$, let $x_{\alpha}^{(n)}$ be the corresponding divided power element in $\operatorname{hy}(G)$. 
Since $\lambda \in X_{1}^{+}$, for $n \geq p, \lambda-n \alpha$ is not a weight in $\Delta(\lambda)$. Thus, $x_{\alpha}^{(n)} \cdot v^{+}=0$ for all $n \geq p$. (For example, as noted in the Introduction, $\Delta(\lambda)$ is obtained by "reduction $\bmod p$ " from the complex irreducible module of high weight $\lambda$, and $\left(\lambda,-\alpha^{\vee}\right)<p$.) Hence, $\exp \left(t x_{\alpha}\right):=\sum_{n \geq 0} t^{n} x_{\alpha}^{(n)}$ satisfies $\exp \left(t x_{\alpha}\right) \cdot v^{+}=\sum_{n=0}^{p-1} t^{n} x_{\alpha}^{(n)} \cdot v^{+} \in E$, for all $t \in k$. (For $n<p, x_{\alpha}^{(n)} \in u(\mathfrak{g})$.) Thus, if $U_{\alpha} \subset B$ is the root subgroup determined by $\alpha, U_{\alpha} \cdot v^{+} \subseteq E$. But $B$ is generated by $T$ and the $U_{\alpha},-\alpha \in \Pi$, so $k B B^{+} \cdot v^{+} \subset E$. Since $B B^{+}$is dense in $G$, we get $k G \cdot v^{+} \subseteq E$, as required.

The following proposition is the $r=1$ case of a result of Lin [31, Thm. 2.7]. It shows that the $\Delta^{\text {red }}$ and $\nabla_{\text {red-construction behaves well with respect to tensor }}$ products. (The proof we give would also work for $r>1$, and seems similar to Lin's proof which does not explicitly use Lemma 1.6, or its $r>1$ analogue.) Let $\mathfrak{g}_{\mathbb{C}}$ be the complex semisimple Lie algebra of the same type as $G$. There is a surjective "Frobenius morphism" $\operatorname{Fr}: U_{\zeta} \rightarrow U\left(\mathfrak{g}_{\mathbb{C}}\right)$, where $U\left(\mathfrak{g}_{\mathbb{C}}\right)$ is the universal enveloping algebra of $\mathfrak{g}_{\mathbb{C}}$. Given any $\mathfrak{g}_{\mathbb{C}}$-module $M, \mathrm{Fr}^{*} M$ denotes the pull-back of $M$ to $U_{\zeta}$. For $\lambda \in X^{+}, \operatorname{Fr}^{*} L_{\mathbb{C}}(\lambda) \cong L_{\zeta}(p \lambda)$, if $L_{\mathbb{C}}(\lambda)$ is the irreducible $\mathfrak{g}_{\mathbb{C}}$-module of high weight $\lambda$.

Proposition 1.7. Suppose $\lambda=\lambda_{0}+p \lambda_{1}$, where $\lambda_{0} \in X_{1}^{+}$and $\lambda_{1} \in X^{+}$. Then $\Delta^{\mathrm{red}}(\lambda)=\Delta^{\mathrm{red}}\left(\lambda_{0}\right) \otimes \Delta\left(\lambda_{1}\right)^{(1)}$ and $\nabla_{\mathrm{red}}(\lambda)=\nabla_{\mathrm{red}}\left(\lambda_{0}\right) \otimes \nabla\left(\lambda_{1}\right)^{(1)}$.

Proof. It suffices to prove the first equality; the second then follows by a dual argument.

Let $v_{0}^{+} \in \Delta^{\text {red }}\left(\lambda_{0}\right)$ and $v_{1}^{+} \in \Delta\left(\lambda_{1}\right)^{(1)}$ be high weight vectors, so that $v_{0}^{+} \otimes v_{1}^{+}$ is a high weight vector in $\Delta^{\text {red }}\left(\lambda_{0}\right) \otimes \Delta\left(\lambda_{1}\right)^{(1)}$. The latter module is the reduction $\bmod p$ of the lattice $\widetilde{L}_{\zeta}^{\min }\left(\lambda_{0}\right) \otimes \widetilde{L}_{\zeta}^{\min }\left(p \lambda_{1}\right)$ in $L_{\zeta}(\lambda)$, and so has the same dimension as $\Delta^{\text {red }}(\lambda)$ since $L_{\zeta}(\lambda) \cong L_{\zeta}\left(\lambda_{0}\right) \otimes L_{\zeta}(p \lambda)$ by the quantum tensor product theorem. Also, we can arrange an inclusion $\widetilde{L}_{\zeta}^{\min }(\lambda) \subseteq \widetilde{L}_{\zeta}^{\min }\left(\lambda_{0}\right) \otimes \widetilde{L}_{\zeta}^{\min }\left(p \lambda_{1}\right)$ with reduction $\bmod p$ of the inclusion, sending a high weight vector of $\Delta^{\text {red }}(\lambda)$ to $v_{0}^{+} \otimes v_{1}^{+}$. Thus, it suffices to prove that $v_{0}^{+} \otimes v_{1}^{+}$generates $\Delta^{\text {red }}\left(\lambda_{0}\right) \otimes \Delta\left(\lambda_{1}\right)^{(1)}$.

Let $\mathfrak{r}=\operatorname{rad}\left(\Delta^{\operatorname{red}}\left(\lambda_{0}\right)\right)$, so that $\Delta^{\operatorname{red}}\left(\lambda_{0}\right) / \mathfrak{r} \cong L\left(\lambda_{0}\right)$. Let $\mu=\mu_{0}+p \mu_{1} \in X^{+}$ with $\mu_{0} \in X_{1}^{+}$and $\mu_{1} \in X^{+}$. Then

$$
\operatorname{Hom}_{G}\left(L\left(\lambda_{0}\right) \otimes \Delta\left(\lambda_{1}\right)^{(1)}, L(\mu)\right) \cong \operatorname{Hom}_{G}\left(\Delta\left(\lambda_{1}\right), L\left(\mu_{1}\right)\right) \otimes \operatorname{Hom}_{G_{1}}\left(L\left(\lambda_{0}\right), L\left(\mu_{0}\right)\right),
$$

since $L(\mu) \cong L\left(\mu_{0}\right) \otimes L\left(\mu_{1}\right)^{(1)}$ by the tensor product theorem. Thus, $L\left(\lambda_{0}\right) \otimes$ $\Delta\left(\lambda_{1}\right)^{(1)}$ has an irreducible head $L\left(\lambda_{0}\right) \otimes L\left(\lambda_{1}\right)^{(1)}$. The image of $v_{0}^{+} \otimes v_{1}^{+}$generates the quotient $\Delta^{\text {red }}\left(\lambda_{0}\right) \otimes \Delta\left(\lambda_{1}\right)^{(1)} / \mathfrak{r} \otimes \Delta\left(\lambda_{1}\right)^{(1)} \cong L\left(\lambda_{0}\right) \otimes \Delta\left(\lambda_{1}\right)^{(1)}$. In other words, if $E$ is the $G$-module generated by $v_{0}^{+} \otimes v_{1}^{+}$in $\Delta^{\text {red }}\left(\lambda_{0}\right) \otimes \Delta\left(\lambda_{1}\right)^{(1)}$, then $E+\mathfrak{r} \otimes$ $\Delta\left(\lambda_{1}\right)^{(1)}=\Delta^{\mathrm{red}}\left(\lambda_{0}\right) \otimes \Delta\left(\lambda_{1}\right)^{(1)}$.

Next, consider $\Delta^{\text {red }}\left(\lambda_{0}\right)$ as a $u(\mathfrak{g}) . T$-module. By Lemma 1.6, $\Delta\left(\lambda_{0}\right)$ is a homomorphic image of $\widehat{Z}_{1}\left(\lambda_{0}\right)$. Hence, $\Delta^{\text {red }}\left(\lambda_{0}\right)$ is also a homomorphic image of $\widehat{Z}_{1}\left(\lambda_{0}\right)$. Since $\widehat{Z}_{1}\left(\lambda_{0}\right)$ has an irreducible head, $\Delta^{\text {red }}\left(\lambda_{0}\right)$ must also, as a $u(\mathfrak{g}) . T$ module, have an irreducible head. Consequently, $\mathfrak{r} \otimes \Delta\left(\lambda_{1}\right)^{(1)}$ is the $u(\mathfrak{g})$.T-radical of $\Delta^{\text {red }}\left(\lambda_{0}\right) \otimes \Delta\left(\lambda_{1}\right)^{(1)}$. (Note that $u(\mathfrak{g})$ acts trivially on the second factor.) Thus, the above discussion and Nakayama's lemma for $u(\mathfrak{g}) \cdot T$-modules imply that $E=$ $\Delta^{\text {red }}\left(\lambda_{0}\right) \otimes \Delta\left(\lambda_{1}\right)^{(1)}$, proving the proposition. 


\section{Grothendieck Groups}

We study the categories $\mathcal{E}^{L}$ and $\mathcal{E}^{R}$ associated to the semisimple group $G$. These categories naturally lead to the "enriched" Grothendieck groups for the category $G$-mod of rational $G$-modules. Our setup follows that introduced in [13]. The tracking of degree information in the derived category of rational $G$-modules provides the main advantage of the enriched Grothendieck groups over the ordinary Grothendieck group of $G$-mod 3

2.1. The categories $\mathcal{E}^{L}, \mathcal{E}^{R}$. Let $\mathcal{D}=D^{b}(G-\bmod )$ be the bounded derived category of the abelian category $G$-mod. The category $G$-mod is fully embedded in $\mathcal{D}$ : if $M \in G$-mod, then $M$ is regarded as a complex in $\mathcal{D}$ concentrated in degree 0 . Let $\mathcal{E}_{0}^{L}$ be the full subcategory of $\mathcal{D}$ consisting of objects isomorphic to finite direct sums of various $\Delta(\lambda)[m]$, where $\lambda \in X^{+}$and $m \equiv l(\lambda) \bmod 2$. Here [1] is the (down) shifting functor in $\mathcal{D}$, and, if $m>0,[m]=\underbrace{[1] \circ \cdots \circ[1]}_{m}$ (with the standard convention if $m<0$ ). If $\mathcal{E}_{i}^{L}$ is defined, for some $i \geq 0$, let $\mathcal{E}_{i+1}^{L} \subset \mathcal{D}$ be the strict, full subcategory consisting of all objects $X$ for which there exists a distinguished triangle $A \rightarrow X \rightarrow B \rightarrow$, with $A, B \in \mathcal{E}_{i}^{L}$. Then $\mathcal{E}_{0}^{L} \subseteq \mathcal{E}_{1}^{L} \subseteq \cdots$. Set

$$
\mathcal{E}^{L}=\mathcal{E}^{L}(G-\bmod )=\bigcup_{i=0}^{\infty} \mathcal{E}_{i}^{L} .
$$

The category $\mathcal{E}^{R}$ is obtained by replacing the $\Delta(\lambda)$ throughout by $\nabla(\lambda)$.

In what follows, $\widehat{\mathcal{E}^{L}}=\mathcal{E}^{L} \oplus \mathcal{E}^{L}[1]$ and $\widehat{\mathcal{E}^{R}}=\mathcal{E}^{R} \oplus \mathcal{E}^{R}[1]$.

Lemma 2.1. (a) $\operatorname{Hom}_{\mathcal{D}}\left(\mathcal{E}^{L}[m], \mathcal{E}^{R}\right)=0$ for any odd integer $m$.

(b) Given $\lambda \in X^{+}$, the following are equivalent:

(1) $L(\lambda) \in \widehat{\mathcal{E}^{L}}$;

(2) $L(\lambda)[-l(\lambda)] \in \mathcal{E}^{L}$

(3) $L(\lambda) \in \widehat{\mathcal{E}^{R}}$

(4) $L(\lambda)[-l(\lambda)] \in \mathcal{E}^{R}$

Proof. (a) is clear from Lemma 1.1, so we prove (b):

$(1) \Longrightarrow(2)$ : Suppose $L(\lambda) \in \widehat{\mathcal{E}^{L}}$, so that $L(\lambda) \in \mathcal{E}^{L} \oplus \mathcal{E}^{L}[1]$. Thus, $L(\lambda)[-l(\lambda)] \in$ $\mathcal{E}^{L}$ or $L(\lambda)[-l(\lambda)] \in \mathcal{E}^{L}[1]$. If $L(\lambda)[-l(\lambda)] \in \mathcal{E}^{L}[1]$, then $\operatorname{Hom}_{\mathcal{D}}\left(L(\lambda)[-l(\lambda)], \mathcal{E}^{R}\right)=$ 0. But

$$
\operatorname{Hom}_{\mathcal{D}}(L(\lambda)[-l(\lambda)], \nabla(\lambda)[-l(\lambda)]) \neq 0,
$$

a contradiction.

$(2) \Longrightarrow(1)$ : trivial.

$(3) \Longleftrightarrow(4)$ : similar to $(1) \Longleftrightarrow(2)$.

$(1) \Longleftrightarrow(3)$ : The category $G$-mod has a duality $D$ fixing irreducible objects and taking each $\Delta(\lambda)$ to $\nabla(\lambda), \lambda \in X^{+}$. It induces a duality $D$ on $\mathcal{D}$ which interchanges $\widehat{\mathcal{E}^{L}}$ and $\widehat{\mathcal{E}^{R}}$. Thus, if (1) holds, so does (3), and conversely.

3 There are other ways to track degree information. For instance, the homological degree can be tracked using the Grothendieck group of a suitable (e.g., Koszul) $\mathbb{Z}$-graded highest weight category; see [15]. In a future paper [37, we show how even a suitable $\mathbb{Z} / 2$-grading is sufficient to track degree information, again using a suitably enriched Grothendieck group structure. However, no such graded structures will be required here. 
Lemma 2.2. In the poset $\left(X^{+}, \uparrow\right)$, let $\Gamma$ be a finite ideal of regular weights. The following statements are equivalent:

(1) $L(\lambda) \in \widehat{\mathcal{E}^{R}}$ for all $\lambda \in \Gamma$;

(2) $L(\lambda) \in \widehat{\mathcal{E}^{L}}$ for all $\lambda \in \Gamma$;

(3) $\operatorname{Ext}_{G}^{n}(L(\lambda), \nabla(\mu)) \neq 0 \Longrightarrow n \equiv l(\lambda)-l(\mu) \bmod 2$, for all $\lambda, \mu \in \Gamma$;

(4) $\operatorname{Ext}_{G}^{n}(\Delta(\lambda), L(\mu)) \neq 0 \Longrightarrow n \equiv l(\lambda)-l(\mu) \bmod 2$, for all $\lambda, \mu \in \Gamma$;

(5) the LCF holds for all $\lambda \in \Gamma$;

(6) the $h L C F$ holds for all $\lambda \in \Gamma$.

Proof. (1) $\Longleftrightarrow$ (2) follows as in the proof of (1) $\Longleftrightarrow$ (3) in Lemma 2.1. Next, $(2) \Longleftrightarrow(3)$, using Lemma 2.1(b) and [13, Thm. 2.4]. Also, (3) $\Longleftrightarrow$ (4), since $\operatorname{Ext}_{G}^{\bullet}(L(\lambda), \nabla(\mu)) \cong \operatorname{Ext}_{G}^{\bullet}(\Delta(\mu), L(\lambda))$. For $(1) \Longleftrightarrow(5) \Longrightarrow(6)$, see [13, Thm. 5.3]. Finally, suppose that (6) holds. Since $P_{y, w}$ is a polynomial in $q=t^{2}$, (3) follows.

Remark 2.3. The proof of the above lemma carries over to the quantum enveloping algebra case. In view of Remark 1.5 and Lemma 2.2(5), all six conditions hold for $L_{\zeta}(\lambda)$.

We also have the following related result, which follows from [13, Thm. 2.4].

Lemma 2.4. Let $M \in G$-mod. Then $M \in \mathcal{E}^{L}$ if and only if $\operatorname{Ext}_{G}^{n}(M, \nabla(\lambda)) \neq 0$ implies that $n \equiv l(\lambda) \bmod 2$ for all $\lambda \in X^{+}$. If the composition factors of $M$ have the form $L\left(w \cdot \lambda^{-}\right)$for some $\lambda^{-} \in \overline{C_{\mathbb{Z}}^{-}}$, then it is sufficient to consider only those $\lambda$ of the form $y \cdot \lambda^{-}$which satisfy $\lambda \leq w \cdot \lambda^{-}$for some composition factor $L\left(w \cdot \lambda^{-}\right)$ in $M$.

2.2. The enriched Grothendieck groups. We continue to work with the category $G$-mod with $\Lambda=X^{+}$, regarded as a poset with respect to the $\leq$partial ordering introduced before. The enriched Grothendieck group $K_{0}^{L}=K_{0}^{L}(G$-mod) is the quotient of the free abelian group on objects of $\widehat{\mathcal{E}}^{L}$ by the subgroup spanned by all relations $X+Z-Y$, where $X \rightarrow Y \rightarrow Z \rightarrow$ is a distinguished triangle with terms $X, Y, Z$ either in $\mathcal{E}^{L}$ or in $\mathcal{E}^{L}[1]$, or a direct sum of two such distinguished triangles. If $X \in \widehat{\mathcal{E}^{L}}$, let $[X]_{L}$ denote its image in $K_{0}^{L}$. The definition implies that $K_{0}^{L}$ is a free module for the ring $\mathbb{Z}\left[t, t^{-1}\right]$ of Laurent polynomials in which $t[X]_{L}=X[-1]_{L}$. Then $K_{0}^{L}$ has basis $\left\{[\Delta(\lambda)]_{L}\right\}_{\lambda \in X^{+}}$.

The discussion in this and the next paragraph is largely taken from [13; see especially Proposition 2.3 there. The right enriched Grothendieck group $K_{0}^{R}=$ $K_{0}^{R}(G-$ mod $)$ is defined similarly, using $\widehat{\mathcal{E}^{R}}$. It is again a free $\mathbb{Z}\left[t, t^{-1}\right]$-module with basis $\left\{[\nabla(\lambda)]_{R}\right\}_{\lambda \in X^{+}}$. Again, $t[X]_{R}:=[X[-1]]_{R}$ for $X \in \widehat{\mathcal{E}^{R}}$. There is a natural bilinear form

$$
\langle,\rangle: K_{0}^{L} \times K_{0}^{R} \rightarrow \mathbb{Z}\left[t, t^{-1}\right]
$$

given by putting

$$
\left\langle[M]_{L},[N]_{R}\right\rangle=\sum_{n} \operatorname{dim} \operatorname{Hom}_{\mathcal{D}}^{n}(M, N) t^{n}
$$

in $\mathbb{Z}\left[t, t^{-1}\right]$ for $M \in \widehat{\mathcal{E}^{L}}, N \in \widehat{\mathcal{E}^{R}}$. Since

$$
\operatorname{Hom}_{\mathcal{D}}^{i}(M[-n], N)=\operatorname{Hom}_{\mathcal{D}}^{i}(M, N[n])=\operatorname{Hom}_{\mathcal{D}}^{n+i}(M, N),
$$


this form is sesquilinear with respect to the action of $\mathbb{Z}\left[t, t^{-1}\right]$. That is,

$$
\left\langle f(t)[M]_{L},[N]_{R}\right\rangle=\left\langle[M]_{L},[N]_{R} f\left(t^{-1}\right)\right\rangle=\left\langle[M]_{L},[N]_{R}\right\rangle f\left(t^{-1}\right)
$$

for any $f(t) \in \mathbb{Z}\left[t, t^{-1}\right]$. By Lemma 1.1, the bases $\left\{[\Delta(\lambda)]_{L}\right\}_{\lambda \in X^{+}}$and $\left\{[\nabla(\lambda)]_{R}\right\}_{\lambda \in X^{+}}$ are naturally "dual" to one another with respect to this pairing.

Thus, if $M \in \widehat{\mathcal{E}^{L}}$ and

$$
[M]_{L}=\sum_{\mu} \bar{p}_{M, \mu}[\Delta(\mu)]
$$

then

$$
p_{M, \mu}=\left\langle[M]_{L},[\nabla(\mu)]_{R}\right\rangle=\sum \operatorname{dim} \operatorname{Hom}_{\mathcal{D}}^{n}(M, \nabla(\mu)) t^{n} .
$$

The Grothendieck group $K_{0}=K_{0}(G-\bmod )$ of $G-\bmod$ is canonically isomorphic to the Grothendieck group of the triangulated category $\mathcal{D}[22$; in practice, we identify the two. If $X \in \mathcal{D}$, let $[X]$ denote its image in $K_{0}$. Then $K_{0}$ is a free abelian group with basis $\{[\Delta(\lambda)]=[\nabla(\lambda)]\}_{\lambda \in X^{+}}$. Regarding $K_{0}$ as a $\mathbb{Z}\left[t, t^{-1}\right]$-module in which $t$ acts as multiplication by -1 , there are $\mathbb{Z}\left[t, t^{-1}\right]$-module morphisms $\pi_{L}$ : $K_{0}^{L} \rightarrow K_{0}$ and $\pi_{R}: K_{0}^{R} \rightarrow K_{0}$ satisfying

$$
\pi_{L} \sum_{\lambda} p_{\lambda}[\Delta(\lambda)]_{L}=\sum_{\lambda} p_{\lambda}(-1)[\Delta(\lambda)]=\pi_{R} \sum_{\lambda} p_{\lambda}[\nabla(\lambda)]_{R}
$$

for $\sum_{\lambda} p_{\lambda}[\Delta(\lambda)]_{L} \in K_{0}^{L}$ and $\sum_{\lambda} p_{\lambda}[\nabla(\lambda)]_{R} \in K_{0}^{R}$. For $M \in \widehat{\mathcal{E}^{L}}$, we have from (2.4.1), (2.4.2) and (2.4.3) that

$$
[M]=\sum_{\mu} \sum_{n}(-1)^{n} \operatorname{dim} \operatorname{Hom}_{\mathcal{D}}^{n}(M, \nabla(\mu))[\Delta(\mu)] .
$$

Lemma 2.5. Assume that the $h L C F$ holds for some $\lambda \in X_{\mathrm{reg}}^{+}$. Then the LCF holds for $\lambda$ and $L(\lambda) \in \widehat{\mathcal{E}^{L}}$.

Proof. Write $\lambda=x \cdot \lambda^{-}$for $\lambda^{-} \in C_{\mathbb{Z}}^{-}$and assume that (1.3.1) holds. Since $P_{y, x}$ is a polynomial in $t^{2}$, it follows that if $\operatorname{Ext}_{G}^{n}\left(L\left(x \cdot \lambda^{-}\right), \nabla\left(y \cdot \lambda^{-}\right) \neq 0\right.$, then $n \equiv$ $l(x)-l(y)=l(\lambda)-l\left(y \cdot \lambda^{-}\right) \bmod 2$. If $\mu \in X^{+}$does not have the form $y \cdot \lambda^{-}$, then $\operatorname{Ext}_{G}^{\bullet}(L(\lambda), \nabla(\mu))=0$. Thus, Lemma 2.4 and Lemma 2.1 imply that $L(\lambda) \in \widehat{\mathcal{E}^{L}}$.

Therefore, (2.4.4) is applicable to $L(\lambda)$, so that

$$
\begin{aligned}
{[L(\lambda)] } & =\sum_{y} \sum_{n}(-1)^{n} \operatorname{dim} \operatorname{Ext}_{G}^{n}\left(L(\lambda), \nabla\left(y \cdot \lambda^{-}\right)\right)\left[\Delta\left(y \cdot \lambda^{-}\right)\right] \\
& =\sum_{y}(-1)^{l(x)-l(y)} \bar{P}_{y, x}(-1)\left[\Delta\left(y \cdot \lambda^{-}\right)\right] \\
& =\sum_{y}(-1)^{l(x)-l(y)} P_{y, x}(-1)\left[\Delta\left(y \cdot \lambda^{-}\right)\right]
\end{aligned}
$$

since $P_{y, x}(-1)=P_{y, x}(1)$. Replacing $[L(\lambda)]$ (resp., $\left[\nabla\left(y \cdot \lambda^{-}\right)\right]$) by ch $L(\lambda)$ (resp., ch $\left.\Delta\left(y \cdot \lambda^{-}\right)=\chi\left(y \cdot \lambda^{-}\right)\right)$, we obtain that $\lambda$ satisfies the LCF. 


\section{HECKE OPERATORS}

Given $\lambda \in X$, there is an exact, additive projection functor $\operatorname{pr}_{\lambda}: G-\bmod \rightarrow$ $G$-mod which assigns to $V \in G$-mod the largest $G$-submodule $\operatorname{pr}_{\lambda} V$, all of whose composition factors have the form $L(\mu)$ for some $\mu \in X^{+} \cap W_{p} \cdot \lambda$. If $\operatorname{Ext}_{G}^{1}(L(\nu), L(\mu))$ $\neq 0$ for $\nu, \mu \in X^{+}$, then $\nu \in W_{p} \cdot \mu$. Hence, given $V \in G-\bmod , V \cong \bigoplus_{\lambda \in \bar{C}_{\mathbb{Z}}^{+}} \operatorname{pr}_{\lambda} V$.

Now let $\lambda, \mu \in \bar{C}_{\mathbb{Z}}^{+}$and let $\nu_{1}$ be the unique element in $W(\mu-\lambda) \cap X^{+}$. Define $T_{\lambda}^{\mu}: G-\bmod \rightarrow G-\bmod$ by putting

$$
T_{\lambda}^{\mu}(V)=\operatorname{pr}_{\mu}\left(L\left(\nu_{1}\right) \otimes \operatorname{pr}_{\lambda} V\right) .
$$

The functor $T_{\lambda}^{\mu}$ is an exact, additive functor with adjoint given by $T_{\mu}^{\lambda}$. As such, $T_{\lambda}^{\mu}$ acts naturally on the category $\mathcal{D}$. By [26, Prop. 7.11], if $\lambda, \mu \in X_{\text {reg }}^{+}, T_{\lambda}^{\mu}$ preserves the full subcategories $\widehat{\mathcal{E}^{L}}$ and $\widehat{\mathcal{E}^{R}}$.

We will also need to work with the category $G_{1} B-\bmod$. For $\lambda \in X$, the projection functor $\widehat{\mathrm{pr}}_{\lambda}: G_{1} B-\bmod \rightarrow G_{1} B-\bmod$ is defined by putting $\widehat{\mathrm{pr}}_{\lambda}(V), V \in G_{1} B-\bmod$, equal to the largest submodule of $V$, all of whose $G_{1} B$-composition factors have the form $\widehat{L}_{1}(\mu)$ for some $\mu \in W_{p} \cdot \lambda$. Then $V=\bigoplus_{\lambda \in \bar{C}_{\mathbb{Z}}^{+}} \widehat{\operatorname{pr}}_{\lambda}(V)$, since given $\lambda, \mu \in X$, if $\operatorname{Ext}_{G_{1} B}^{1}(\widehat{L}(\nu), \widehat{L}(\mu)) \neq 0$, then $\nu \in W_{p} \cdot \mu$ [26, Prop. 9.20]. In addition,

$$
\operatorname{res}_{G_{1} B}^{G} \circ \operatorname{pr}_{\lambda}=\widehat{\operatorname{pr}}_{\lambda} \circ \operatorname{res}_{G_{1} B}^{G} .
$$

As discussed in [26, Ch. 9], given $\lambda, \mu \in \bar{C}_{\mathbb{Z}}^{+}$, the translation functor $\widehat{T}_{\lambda}^{\mu}: G_{1} B-\bmod$ $\rightarrow G_{1} B-\bmod$ is defined on $V \in G_{1} B-\bmod$ by

$$
\widehat{T}_{\lambda}^{\mu}(V)=\widehat{\mathrm{pr}}_{\mu}\left(\widehat{L}_{1}\left(\nu_{1}\right) \otimes \widehat{\mathrm{pr}}_{\lambda} V\right)
$$

where $\nu_{1} \in X^{+}$is as in (3.0.1).

While the translation functors have been defined for $G-\bmod$ and $G_{1} B$-mod, they carry over with the same definition for the larger categories $G$-Mod and $G_{1} B$-Mod.

For any $\lambda \in X$, we have

$$
\operatorname{pr}_{\lambda} \circ \operatorname{ind}_{G_{1} B}^{G}=\operatorname{ind}_{G_{1} B}^{G} \circ \widehat{p r}_{\lambda} .
$$

In fact, given $\tau=p \tau_{1}+\tau_{0} \in X$, with $\tau_{0} \in X_{1}^{+}$, Lemma $1.2 \operatorname{implies}$ that $\operatorname{ind}_{G_{1} B}^{G} \widehat{L}(\tau)$ $\cong \nabla\left(\tau_{1}\right)^{(1)} \otimes L\left(\tau_{0}\right)$. Here we interpret $\nabla\left(\tau_{1}\right)=0$ if $\tau_{1} \notin X^{+}$. If $\tau_{1} \in X^{+}$, the composition factors $L(\xi)$ of $\nabla\left(\tau_{1}\right)^{(1)} \otimes L\left(\tau_{0}\right)$ satisfy $\xi \in W_{p} \cdot \tau$. It follows by induction on the length of a composition series that, given $V \in G-\bmod$, and $\nu, \mu \in \bar{C}_{\mathbb{Z}}$ with $\nu \notin W_{p} \cdot \mu$, we have $\operatorname{pr}_{\nu} \operatorname{ind}_{G_{1} B}^{G} \widehat{\operatorname{pr}}_{\mu} V=0$. Hence,

$$
\begin{aligned}
\operatorname{pr}_{\lambda} \circ \operatorname{ind}_{G_{1} B}^{G} & =\operatorname{pr}_{\lambda} \circ \operatorname{ind}_{G_{1} B}^{G} \circ \widehat{p r}_{\lambda} \\
& =\operatorname{ind}_{G_{1} B}^{G} \circ \widehat{p r}_{\lambda} .
\end{aligned}
$$

Now (3.0.4) follows directly.

For $\omega \in X$, let $t_{p \omega} \in \widetilde{W}_{p}$ by translation by the weight $p \omega$. We also let $t_{p \omega}$ denote the functor $G_{1} B-\bmod \rightarrow G_{1} B-\bmod$ given by $V \mapsto p \omega \otimes M$. We have the following useful result.

Lemma 3.1. (a) Let $\lambda, \mu \in \bar{C}_{\mathbb{Z}}^{-}$and $\omega \in X$. Let $n$ be the image of $t_{p \omega}$ under the quotient map $\widetilde{W}_{p} \rightarrow \widetilde{W}_{p} / W_{p}=N$. Then

$$
\widehat{T}_{\lambda}^{\mu} \circ t_{p \omega}=t_{p \omega} \circ \widehat{T}_{n^{-1} \cdot \lambda}^{n^{-1} \cdot \mu} .
$$


(b) Let $\nu, \nu^{\prime} \in \bar{C}_{\mathbb{Z}}^{-}$. Then

$$
\left\{\begin{array}{l}
T_{\nu}^{\nu^{\prime}} \circ \operatorname{ind}_{G_{1} B}^{G}=\operatorname{ind}_{G_{1} B}^{G} \circ \widehat{T}_{\nu}^{\nu^{\prime}}, \\
T_{\nu}^{\nu^{\prime}} \circ \operatorname{res}_{G_{1} B}^{G}=\operatorname{res}_{G_{1} B}^{G} \circ \widehat{T}_{\nu}^{\nu^{\prime}} .
\end{array}\right.
$$

(c) Suppose that $p \geq h$ and that $\lambda, \lambda^{\prime} \in X^{+}$are restricted and lie in the same alcove $C$. Suppose that $\nu \in X^{+}$and $L(\lambda) \otimes \nabla(\nu)^{(1)} \in \widehat{\mathcal{E}^{R}}$. Then $L\left(\lambda^{\prime}\right) \otimes \nabla(\nu)^{(1)} \in$ $\widehat{\mathcal{E}^{R}}$. Dually, if $L(\lambda) \otimes \Delta(\nu)^{(1)} \in \widehat{\mathcal{E}^{L}}$, then $L\left(\lambda^{\prime}\right) \otimes \Delta(\nu)^{(1)} \in \widehat{\mathcal{E}^{L}}$.

Proof. We first prove (a). If $\delta \in \bar{C}_{\mathbb{Z}}^{+}$, then $\xi \in W_{p} \cdot \delta$ implies that $p \omega+\xi \in W_{p} \cdot(n \cdot \delta)$. Therefore, using the notation of (3.0.1),

$$
\begin{aligned}
\widehat{T}_{\lambda}^{\mu} \circ t_{p \omega}(V) & \cong \widehat{\mathrm{pr}}_{\mu}\left(\widehat{\mathrm{pr}}_{\lambda}\left(t_{p \omega}(V)\right) \otimes \widehat{L}_{1}\left(\nu_{1}\right)\right) \\
& \cong \widehat{\mathrm{pr}}_{\mu}\left(t_{p \omega} \circ \widehat{\mathrm{pr}}_{n^{-1} \cdot \lambda}(V) \otimes \widehat{L}_{1}\left(\nu_{1}\right)\right) \\
& \cong t_{p \omega} \circ \widehat{\mathrm{pr}}_{n^{-1} \cdot \mu}\left(\widehat{\mathrm{pr}}_{n^{-1} \cdot \lambda}(V) \otimes \widehat{L}_{1}\left(\nu_{1}\right)\right)
\end{aligned}
$$

On the other hand, write $n^{-1}=x t_{-p \omega}$, for $x \in W$. Then

$$
\begin{aligned}
n^{-1} \cdot \mu-n^{-1} \cdot \lambda & =x t_{-p \omega} \cdot \mu-x t_{-p \omega} \cdot \lambda \\
& =x(\mu)-x \cdot p \omega-x(\lambda)+x \cdot p \omega \\
& =x(\mu-\lambda) .
\end{aligned}
$$

The desired formula in (a) follows by combining (3.1.1) and (3.1.2) with the definition of the translation functors.

The first part of assertion (b) follows immediately from (3.0.4), using the definitions (3.0.1) and (3.0.3) of translation. The second part is adjoint to the first part.

To see (c), let $\tau=\lambda+p \nu$ and $\tau^{\prime}=\lambda^{\prime}+p \nu$. Clearly, $\tau$ and $\tau^{\prime}$ are both regular weights lying in the same alcove. At the level of $G_{1} B-\bmod$, the translation functor $\widehat{T}_{\tau}^{\tau^{\prime}}$ takes $\widehat{L}_{1}(\tau)$ to $\widehat{L}_{1}\left(\tau^{\prime}\right)$. (See [26, Prop. 9.19(4)].) Hence, by (1),

$$
\begin{aligned}
T_{\tau}^{\tau^{\prime}}\left(L(\lambda) \otimes \nabla(\nu)^{(1)}\right) & \cong T_{\tau}^{\tau^{\prime}}\left(\operatorname{ind}_{G_{1} B}^{G} L_{1}(\tau)\right) \\
& \cong \operatorname{ind}_{G_{1} B}^{G}\left(\widehat{T}_{\tau}^{\tau^{\prime}}\left(\widehat{L}_{1}(\tau)\right)\right) \\
& \cong \operatorname{ind}_{G_{1} B}^{G}\left(\widehat{L}_{1}\left(\tau^{\prime}\right)\right) \\
& \cong L\left(\lambda^{\prime}\right) \otimes \nabla(\nu)^{(1)}
\end{aligned}
$$

Since the translation functors define an equivalence between the block containing $L(\tau)$ and that containing $L\left(\tau^{\prime}\right)$, it follows that $L\left(\lambda^{\prime}\right) \otimes \nabla(\nu)^{(1)} \in \widehat{\mathcal{E}^{R}}$ provided that $L(\lambda) \otimes \nabla(\nu)^{(1)} \in \widehat{\mathcal{E}^{R}}$. This proves the first assertion in (b), and the second follows dually.

Now assume that $p \geq h$. We will require a variation of wall-crossing functors [26. II.7.2.1]. For each simple reflection $s \in S_{p}$, fix $\mu=\mu_{s} \in \overline{C_{\mathbb{Z}}^{-}}$to lie on the $s$-face of $\overline{C^{-}}$. We will define a functor $\theta_{s}: G-\bmod \rightarrow G-$ mod. Let $\mathcal{B}$ be a block in $G$-mod. If the irreducible modules $L(\tau)$ contained in $\mathcal{B}$ have singular high weights $\tau$, we set $\theta_{s} \equiv 0$ on $\mathcal{B}$. Otherwise, suppose that the irreducible modules in $\mathcal{B}$ have high weights in $W_{p} \cdot \tau$ for $\tau \in C^{-}$; then we put $\theta_{s}(M)=T_{\mu}^{\tau} \circ T_{\tau}^{\mu}$ on $\mathcal{B}$. Since $\theta_{s}$ is defined on each block $\mathcal{B}$, it is defined on $G-\bmod$. 
For any $M \in G-\bmod$, the adjointness of the $T_{\mu}^{\lambda}$ and $T_{\lambda}^{\mu}$ defines morphisms

$$
M \stackrel{\delta(M)}{\rightarrow} \theta_{s} M \stackrel{\epsilon(M)}{\longrightarrow} M
$$

For certain $M \in G$-mod, (3.1.3) defines a complex (with $\Theta_{s} M$ of degree 0), and hence defines an element, denoted $\beta_{s} M$, in the derived category $\mathcal{D}=D^{b}(G-\bmod )$. Thus, $\beta_{s} M$ is defined when $M=L(\lambda), \Delta(\lambda)$, or $\nabla(\lambda)$. When $M=L(\lambda)$ and $\lambda<\lambda s, \beta_{s} L(\lambda)$ is a complex with cohomology concentrated in degree 0 . (If $\lambda s<\lambda$, then $\theta_{s} L(\lambda)=0$.)

At the level of $G_{1} B$-mod, there are similar constructions. On modules with composition factors $\widehat{L}_{1}(\lambda)$ with $\lambda \in W_{p} \cdot \tau$, where $\tau \in C^{-}$, then $\widehat{\theta}_{s} \equiv \widehat{T}_{\mu}^{\tau} \circ \widehat{T}_{\tau}^{\mu}$. In this case, we have an analogous sequence

$$
M \stackrel{\widehat{\delta}(M)}{\rightarrow} \widehat{\theta}_{s} M \stackrel{\widehat{\epsilon}(M)}{\rightarrow} M
$$

in $G_{1} B$-mod for any $G_{1} B$-module $M$. In some cases, (3.1.4) is a complex. In this case, let $\widehat{\beta}_{s}(M)$ denote the corresponding complex in $D^{b}\left(G_{1} B\right.$-mod). For example, suppose $\lambda$ is a regular weight such that $\lambda<\lambda s$. Then the complex $\widehat{\beta}_{s}\left(\widehat{L}_{1}(\lambda)\right)$ is defined, and it has cohomology concentrated in degree 0 . Although the functors $\theta_{s}, \widehat{\theta}_{s}$ have been defined on the categories $G$-mod and $G_{1} B$-mod of finite-dimensional modules, the definitions make sense for the categories $G$-Mod and $G_{1} B$-Mod.

Theorem 3.2. Assume that $p \geq h$ and let $s \in S_{p}$.

(a) For $n \in \mathbb{N}$, we have $\theta_{s} \circ R^{n} \operatorname{ind}_{G_{1} B}^{G}=R^{n} \operatorname{ind}_{G_{1} B}^{G} \circ \widehat{\theta}_{s}$ for all $s \in S_{p}$.

(b) Let $\lambda \in X_{\text {reg }}^{+}$satisfy $\lambda<\lambda$ s. Identify $\widehat{\beta}_{s} \widehat{L}_{1}(\lambda)$ with its cohomology. For $n>0$,

$$
R^{n} \operatorname{ind}_{G_{1} B}^{G} \widehat{\beta}_{s}\left(\widehat{L}_{1}(\lambda)\right)=0 .
$$

Proof. We first prove (a). Because $\theta_{s}$ and $\widehat{\theta}_{s}$ are exact functors preserving injective modules (in the categories $G$-Mod and $G_{1} B$-Mod), it suffices to prove (a) when $n=0$. But the $n=0$ case follows immediately from Lemma 3.1(a).

Now we prove (b). Write $\lambda=\lambda_{0}+p \lambda_{1}$, where $\lambda_{0} \in X_{1}^{+}$. The definition of $\theta_{s}$, together with [26, (9.19(4))], implies that $\widehat{\theta}_{s} \widehat{L}_{1}(\lambda) \cong p \lambda_{1} \otimes M$, where $M$ is a rational $G$-module (see Lemma (3.1) (a) and (3.0.2). In fact, $M=\left.\theta_{s^{\prime}} L\left(\lambda_{0}\right)\right|_{G_{1} B} \cong \widehat{\theta}_{s^{\prime}} \widehat{L}_{1}\left(\lambda_{0}\right)$ for some $s^{\prime} \in S_{p}$. It follows that $\widehat{\beta}_{s} \widehat{L}_{1}(\lambda)$ has the form $p \lambda_{1} \otimes N$ for a rational $G$-module $N$. (Tensor the defining sequence for $\widehat{\beta}_{s} \widehat{L}_{1}(\lambda)$ with $-p \lambda_{1}$, and use the fact that $B$-module morphisms of $G$-modules are always $G$-module morphisms.) Therefore, $R^{n} \operatorname{ind}_{G_{1} B}^{G} \widehat{\beta}_{s} \widehat{L}_{1}(\lambda) \cong\left(R^{n} \operatorname{ind}_{B}^{G} \lambda_{1}\right)^{(1)} \otimes N$ by Lemma 1.2, which vanishes if $n>0$ by Kempf's theorem.

Corollary 3.3. Let $\omega, \lambda \in X^{+}$with $\lambda \in X_{\mathrm{reg}}^{+}$. Assume that $s \in S_{p}$ so that $\lambda+p \omega<(\lambda+p \omega) s$. (Equivalently, $\lambda<\lambda s^{\prime}$, where $s^{\prime}=n \cdot \lambda$ with $n$ the image of $t_{p \omega}$ in $N=\widetilde{W}_{p} / W_{p}$.) Then $\beta_{s}$ is defined for $M=\Delta(\omega)^{(1)} \otimes L(\lambda)$, and $\beta_{s} M$ has cohomology concentrated in degree 0 .

Proof. In the category $G_{1} B$-mod, (3.1.4) defines a complex

$$
\widehat{L}_{1}(p \omega+\lambda) \rightarrow \widehat{\theta}_{s} \widehat{L}_{1}(p \omega+\lambda) \rightarrow \widehat{L}_{1}(p \omega+\lambda)
$$

which has cohomology concentrated in degree 0 . In fact, this cohomology has the form $p \omega \otimes N$ for a rational $G$-module $N$. The corollary now follows from Theorem 
3.2 by applying induction $\operatorname{ind}_{G_{1} B}^{G}$ to this sequence. Note that $R^{1} \operatorname{ind}_{G_{1} B}^{G}$ vanishes on $\widehat{L}_{1}(p \omega+\lambda)$ and well as on $p \omega \otimes N=0$.

Lemma 3.4. Suppose that $M \in G$-mod is such that (3.1.3) defines a complex with cohomology concentrated in degree 0 and that $M \in \widehat{\mathcal{E}^{L}}$. Then for any $\nu \in$ $X_{\mathrm{reg}}^{+}, \operatorname{Hom}_{\mathcal{D}}^{n}\left(\beta_{s} M, \nabla(\nu)\right) \cong \operatorname{Hom}_{\mathcal{D}}^{n}\left(M, \beta_{s} \nabla(\nu)\right)$. If, in addition, all the composition factors of $M$ have regular high weights, then $\beta_{s} M \in \widehat{\mathcal{E}^{L}}$ also.

Proof. The argument given in [15, Thm. 4.8.3] applies (with $M$ replacing the irreducible object $L(\zeta)$ introduced above (4.8.3.2) there - the irreduciblity is not used).

\section{TWisted MOdules}

We consider whether the twisted modules $\Delta(\lambda)^{(1)}$ (resp., $\left.\nabla(\lambda)^{(1)}\right), \lambda \in X^{+}$, belong to $\widehat{\mathcal{E}^{L}}$ (resp., $\left.\widehat{\mathcal{E}^{R}}\right)$. By Proposition 1.7, $\Delta^{\text {red }}(p \lambda) \cong \Delta(\lambda)^{(1)}$ and $\nabla_{\text {red }}(p \lambda) \cong$ $\nabla(\lambda)^{(1)}$ for all $\lambda \in X^{+}$.

An easy spectral sequence argument shows that for $\mu \in X^{+}$, if $H^{\bullet}\left(G_{1}, \nabla(\mu)\right) \neq 0$, then $\mu \in \widetilde{W}_{p} \cdot 0$. (One can also argue from the linkage principle [26, II.9.1.9(1)].) The following result provides more precise information on such cohomology modules. The restriction that $p>h$ is required in order to use 30. Write $\prod_{\alpha \in \Phi^{+}}\left(1-t e^{\alpha}\right)^{-1}=$ $\sum_{\sigma \in X} \sum_{n=0}^{\infty} \mathfrak{p}_{n}(\sigma) e^{\sigma} t^{n}$.

Lemma 4.1. Assume that $p>h$.

(a) For any nonnegative integer $n$ and $\mu \in X$, the rational $G$-module $H^{n}\left(G_{1}, \nabla(\mu)\right)^{(-1)}$ is nonzero only if $\mu=w \cdot 0+p \xi \in \widetilde{W}_{p} \cdot 0 \cap X^{+}(w \in W$, $\xi \in X)$ with $n \equiv l(w) \bmod 2$. (Also, $w \cdot 0+p \xi \in X^{+}$implies $\xi \in X^{+}$.)

(b) Assume $H^{n}\left(G_{1}, \nabla(\mu)\right)^{(-1)} \neq 0$. Then $H^{n}\left(G_{1}, \nabla(\mu)\right)^{(-1)}$ has a G-filtration with sections $\nabla(\lambda), \lambda \in X^{+}$. The multiplicity of $\nabla(\lambda)$ as a section in such a $\nabla$-filtration is given by

$$
\begin{aligned}
& {\left[H^{n}\left(G_{1}, \nabla(\mu)\right)^{(-1)}: \nabla(\lambda)\right]} \\
& \quad= \begin{cases}\sum_{x \in W}(-1)^{l(x)} \mathfrak{p}_{\frac{n-l(w)}{2}}(x \cdot \lambda-\xi) \text { if } n \equiv l(w) \bmod 2 ; \\
0, & \text { otherwise. }\end{cases}
\end{aligned}
$$

Proof. For $w \in W$ and $\alpha \in \Pi,\left|\left(w \cdot 0, \alpha^{\vee}\right)\right|=\left|\left(w \rho, \alpha^{\vee}\right)-1\right| \leq h$. Hence, if $\mu=w \cdot 0+p \xi \in X^{+}$with $w \in W$, then the restriction on $p$ forces $\xi \in X^{+}$.

Let $\mathfrak{u}$ be the Lie algebra of the unipotent radical $R_{u}(B)$ of $B$. A root subgroup $U_{\alpha}$ is contained in $B^{-}$if and only if $-\alpha \in \Phi^{+}$. By [30, Thm. 2], $H^{n}\left(G_{1}, \nabla(\mu)\right)^{(-1)} \cong$ $H^{0}\left(G / B, S^{\frac{n-l(w)}{2}}\left(\mathfrak{u}^{*}\right) \otimes \xi\right)$ for any $n \geq 0$, where the right-hand side is interpreted as 0 if $n \not \equiv l(w) \bmod 2$. For $\gamma \in X$, if there exists $x \in W$ such that $\gamma=x \cdot \gamma^{+}$for $\gamma^{+} \in X^{+}$, then by (1.1.3), $\chi(\gamma)=(-1)^{l(x)} \operatorname{ch} \Delta\left(\gamma^{+}\right)$. Otherwise, $\gamma$ is $W$-singular, and $\chi(\gamma)=0$. By [30, Thm. 2], $\operatorname{ch} H^{0}\left(G / B, S^{\frac{n-l(w)}{2}}\left(\mathfrak{u}^{*}\right) \otimes \xi\right)=\chi\left(S^{\frac{n-l(w)}{2}}\left(\mathfrak{u}^{*}\right) \otimes \xi\right)$, and [30. Thm. 8] implies that $H^{0}\left(G / B, S^{\frac{n-l(w)}{2}}\left(\mathfrak{u}^{*}\right) \otimes \xi\right)$ has a $\nabla$-filtration. Because $\chi(M)=\chi(N)+\chi(M / N)$, whenever $N$ is a $B$-submodule of $M$, the multiplicity of $\nabla(\lambda)$ as a section in such a $\nabla$-filtration is given by (4.1.1).

By Lemma1.1(b), for any $\lambda \in X^{+}$, ch $\Delta(\lambda)^{(1)}=\sum_{\mu \in X^{+}} p_{\mu, \Delta(\lambda)^{(1)}}(-1) \operatorname{ch} \Delta(\mu)$, where $p_{\mu, \Delta(\lambda)(1)}$ is the Poincaré polynomial defined in (1.0.4) for $M=\Delta(\lambda)^{(1)}$. 
The following result should hold for $p=h$. The third author and a University of Virginia undergraduate, Mark Rawls, have checked this result empirically for the case $p=h=7$. The verification was obtained in the course of a general program to implement the proposition and the proof of Theorem 6.8 as a new algorithm for calculating the Kazhdan-Lusztig polynomials (for affine Weyl groups) appearing in the LCF.

Proposition 4.2. Assume that $p>h$ and that $\lambda, \mu \in X^{+}$. Then $p_{\mu, \Delta(\lambda)^{(1)}}=0$ unless $\mu=w \cdot 0+p \xi, w \in W, \xi \in X$. In this case,

$$
p_{\mu, \Delta(\lambda)^{(1)}}=\sum_{n=0}^{\infty} \sum_{x \in W}(-1)^{l(x)} \mathfrak{p}_{\frac{n-l(w)}{2}}(x \cdot \lambda-\xi) t^{n},
$$

where the sum is restricted to those integers $n$ such that $n \equiv l(w) \bmod 2$.

Proof. There is a Hochschild-Serre spectral sequence

$$
E_{2}^{s, t}=\operatorname{Ext}_{G}^{s}\left(\Delta(\lambda), H^{t}\left(G_{1}, \nabla(\mu)\right)^{(-1)}\right) \Rightarrow \operatorname{Ext}_{G}^{s+t}\left(\Delta(\lambda)^{(1)}, \nabla(\mu)\right) .
$$

If $H^{t}\left(G_{1}, \nabla(\mu)\right) \neq 0$, then Lemma 4.1 implies that $\mu=w \cdot 0+p \xi$ and $t \equiv l(w) \bmod$ 2. Also, $H^{t}\left(G_{1}, \nabla(\mu)^{(-1)}\right)$ has a $\nabla$-filtration, so in (4.2.1), $E_{2}^{s, t}=0$ unless $s=0$ by Lemma 1.1(a). Thus, using Lemma 4.1

$$
\begin{aligned}
\operatorname{dim} \operatorname{Ext}_{G}^{n}\left(\Delta(\lambda)^{(1)}, \nabla(\mu)\right) & =\operatorname{dim} \operatorname{Hom}_{G}\left(\Delta(\lambda), H^{n}\left(G_{1}, \nabla(\mu)\right)^{(-1)}\right) \\
& =\left[H^{n}\left(G_{1}, \nabla(\mu)\right)^{(-1)}: \nabla(\lambda)\right] .
\end{aligned}
$$

Now apply 4.1.1.

Now we can answer the question posed by this section.

Theorem 4.3. Assume that $p>h$. Let $\lambda \in X^{+}$. Then

$\Delta^{\mathrm{red}}(p \lambda)[-l(p \lambda)]=\Delta(\lambda)^{(1)}[-l(p \lambda)] \in \mathcal{E}^{L}$ and $\nabla(\lambda)^{(1)}[-l(p \lambda)]=\nabla(\lambda)^{(1)}[-l(\lambda)] \in \mathcal{E}^{R}$.

$\left(\right.$ Here $l(p \lambda):=l\left(t_{p \lambda}\right)=\sum_{\alpha \in \Phi^{+}}\left(\lambda, \alpha^{\vee}\right)$.)

Proof. We prove that $\Delta(\lambda)^{(1)}\left[-l\left(t_{p \lambda}\right)\right] \in \mathcal{E}^{L}$; that $\nabla(\lambda)^{(1)}\left[-l\left(t_{p \lambda}\right)\right] \in \mathcal{E}^{R}$ is handled similarly. The composition factors $L(\tau)$ of $\Delta(\lambda)^{(1)}$ satisfy $\tau \in \widetilde{W}_{p} \cdot 0$ and $l(\tau) \equiv$ $l(p \lambda):=l\left(t_{p \lambda}\right) \bmod 2$. We must show that if $\mu \in X^{+}$, then

$$
\operatorname{Ext}_{G}^{n}\left(\Delta(\lambda)^{(1)}, \nabla(\mu)\right) \neq 0 \Longrightarrow n \equiv l(p \lambda)-l(\mu) \bmod 2 .
$$

We must have $\mu=w \cdot 0+p \xi$ and $n \equiv l(w) \bmod 2$. So, to conclude the proof, we must determine that $l(p \lambda) \equiv l(p \xi) \bmod 2$. If $\operatorname{Ext}_{G}^{n}\left(\Delta(\lambda)^{(1)}, \nabla(\mu)\right) \neq 0$, then $p \lambda$ and $\mu=w \cdot 0+p \xi$ belong to the same $W_{p}$-linkage class. Hence, $p \lambda-p \xi \in \mathbb{Z} \Phi$. Since $p>h, X / \mathbb{Z} \Phi$ has no $p$-torsion, so $p \lambda=p \xi+p \delta$, with $\delta \in \mathbb{Z} \Phi$. Then $t_{p \lambda}=t_{p \xi} t_{p \delta}$. Since $l\left(t_{p \delta}\right)$ is even, $l(p \lambda) \equiv l(p \xi) \bmod 2$, as required.

Corollary 4.4. Assume that $p>h$. Suppose that $\lambda \in X^{+}$and $\Delta(\lambda) \cong L(\lambda)$. Then $L(\lambda)^{(1)}[-l(p \lambda)]=L(p \lambda)[-l(p \lambda)] \in \mathcal{E}^{L} \cap \mathcal{E}^{R}$.

In type $A_{n-1}$ (i.e., $G=S L_{n}(k)$ ), there is a determination of all $\lambda$ for which $\Delta(\lambda)=L(\lambda)$, given in [26, $(8.21)]$. 


\section{QUANTUM GROUPS AND SOME INTEGRAL REPRESENTATION THEORY}

We consider when $\lambda \in X_{\text {reg }}^{+}$satisfies the hLCF, as defined in (1.3.1). We will say that $X \in \mathcal{D}$ satisfies the $\widehat{E}^{L}$ (resp., $\widehat{E}^{R}$ ) condition provided that $X \in \widehat{\mathcal{E}^{L}}$ (resp., $X \in \widehat{\mathcal{E}^{R}}$ ). Given $\lambda \in X_{\text {reg }}^{+}$, write $\lambda \in \mathrm{LCF}$ (resp., $\lambda \in \mathrm{hLCF}, \lambda \in \widehat{E}^{L}$, $\lambda \in \widehat{E}^{R}$ ) provided that $L(\lambda)$ satisfies the LCF (resp., the hLCF, the $\widehat{E}^{L}$ condition, $\widehat{E}^{R}$ condition). Of course, $\lambda \in \widehat{E}^{L} \Longleftrightarrow \lambda \in \widehat{E}^{R}$.

Theorem 5.1. For $\lambda \in X_{\mathrm{reg}}^{+}, \lambda \in \mathrm{hLCF}$ if and only if $\lambda \in \mathrm{LCF}$ and $\lambda \in \widehat{E}^{L}$.

Proof. First, suppose that $\lambda \in \mathrm{hLCF}$. Write $\lambda=w \cdot \lambda^{-}, \lambda^{-} \in \overline{C_{\mathbb{Z}}^{-}}$. If $\mu \notin W_{p} \cdot \lambda^{-}$, then $\operatorname{Ext}_{G}^{\bullet}(L(\lambda), \nabla(\mu))=0$ by the linkage principle. Hence, if the hLCF holds, then for $\mu=y \cdot \lambda^{-}$, we have, by (1.3.1) that $p_{y \cdot \lambda^{-}, L\left(w \cdot \lambda^{-}\right)}(-1)=(-1)^{l(w)-l(y)} \bar{P}_{y, w}(-1)=$ $(-1)^{l(w)-l(y)} P_{y, w}(-1)$. Therefore, Lemma 1.1(b) implies that $\operatorname{ch} L(\lambda)=\chi_{\mathrm{KL}}(\lambda)$, so that $\lambda \in \mathrm{LCF}$. Also, since $P_{y, w}$ is a polynomial in $q=t^{2}$, the validity of (1.3.1) implies that, if $\operatorname{Ext}_{G}^{n}(L(\lambda), \nabla(\mu)) \neq 0$, then $l(\lambda)-l(\mu) \equiv n \bmod 2$. So, $L(\lambda)[-l(\lambda)] \in \mathcal{E}^{L}$ and $\lambda \in \widehat{E}^{L}$.

To prove the reverse direction, assume that $\lambda \in \mathrm{LCF}$ and $\lambda \in \widehat{E}^{L}$. Then $\lambda \in$ hLCF, provided that

$$
\operatorname{dim} \operatorname{Ext}_{U_{\zeta}}^{n}\left(L_{\zeta}(\lambda), \nabla_{\zeta}(\mu)\right)=\operatorname{dim} \operatorname{Ext}_{G}^{n}(L(\lambda), \nabla(\mu))
$$

holds for any $\mu \in X_{\text {reg }}^{+}$and all nonnegative integers $n$. The left-hand side of (5.1.1)

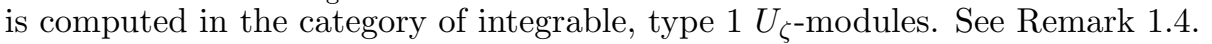

Write $\widetilde{L}_{\zeta}(\lambda)=\widetilde{L}_{\zeta}^{\min }(\lambda)=\widetilde{U}_{\zeta} \cdot v^{+}$and choose an admissible lattice $\widetilde{\nabla}_{\zeta}(\mu)$ for $\nabla_{\zeta}(\mu)$. We can assume that $\widetilde{\nabla}_{\zeta}(\mu) / \pi \widetilde{\nabla}_{\zeta}(\mu) \cong \nabla(\mu)$; see [20, p. 159] (which makes use of results of [5]). Because the LCF holds for $\lambda, L(\lambda) \cong \widetilde{L}_{\zeta}(\lambda) / \pi \widetilde{L}_{\zeta}(\lambda)$. Thus, we have a short exact sequence $0 \rightarrow \widetilde{\nabla}_{\zeta}(\mu) \stackrel{p}{\rightarrow} \widetilde{\nabla}_{\zeta}(\mu) \rightarrow \nabla(\mu) \rightarrow 0$. By (1.5.5), $\operatorname{Ext}_{\widetilde{U}_{\zeta}}^{\bullet}\left(\widetilde{L}_{\zeta}(\lambda), \nabla(\mu)\right) \cong \operatorname{Ext}_{G}^{\bullet}(L(\lambda), \nabla(\mu))$.

Therefore, by the long exact sequence of Ext for $\operatorname{Hom}_{\widetilde{U}_{\zeta}}\left(\widetilde{L}_{\zeta}(\lambda),-\right)$, we obtain, for any nonnegative integer $n$, a long exact sequence

$$
\cdots \rightarrow \operatorname{Ext}_{\widetilde{U}_{\zeta}}^{n}\left(\widetilde{L}_{\zeta}(\lambda), \widetilde{\nabla}_{\zeta}(\mu)\right) \stackrel{\pi}{\rightarrow} \operatorname{Ext}_{\widetilde{U}_{\zeta}}^{n}\left(\widetilde{L}_{\zeta}(\lambda), \widetilde{\nabla}_{\zeta}(\mu)\right) \rightarrow \operatorname{Ext}_{G}^{n}(L(\lambda), \nabla(\mu)) \rightarrow \cdots
$$

But, by hypothesis, $L(\lambda) \in \widehat{\mathcal{E}^{L}}$, while $L_{\zeta}(\lambda)$ belongs to the analogous category for $U_{\zeta}$. If $n \not \equiv l(\lambda)-l(\mu) \bmod 2$, then $\operatorname{Ext}_{\widetilde{U}_{\zeta}}^{n}\left(\widetilde{L}_{\zeta}(\lambda), \widetilde{\nabla}_{\zeta}(\mu)\right) \stackrel{\pi}{\rightarrow} \operatorname{Ext}_{\widetilde{U}_{\zeta}}^{n}\left(\widetilde{L}_{\zeta}(\lambda), \widetilde{\nabla}_{\zeta}(\mu)\right)$ is surjective. Since $\operatorname{Ext}_{\widetilde{U}_{\zeta}}^{n}\left(\widetilde{L}_{\zeta}(\lambda), \widetilde{\nabla}_{\zeta}(\mu)\right)$ is a finite $\mathcal{O}$-module, $\operatorname{Ext}_{\widetilde{U}_{\zeta}}^{n}\left(\widetilde{L}_{\zeta}(\lambda), \widetilde{\nabla}_{\zeta}(\mu)\right)=$ 0 in this case. It follows that, when $n \equiv l(\lambda)-l(\mu) \bmod 2$, the above long exact sequences provide a short exact sequence $0 \rightarrow \operatorname{Ext}_{\widetilde{U}_{\zeta}}^{n}\left(\widetilde{L}_{\zeta}(\lambda), \widetilde{\nabla}_{\zeta}(\mu)\right) \stackrel{\pi}{\rightarrow} \operatorname{Ext}_{\widetilde{U}_{\zeta}}^{n}\left(\widetilde{L}_{\zeta}(\lambda)\right.$, $\left.\widetilde{\nabla}_{\zeta}(\mu)\right) \rightarrow \operatorname{Ext}_{G}^{n}(L(\lambda), \nabla(\mu)) \rightarrow 0$. If $n$ does not satisfy the congruence $n \equiv l(\lambda)-l(\mu) \bmod 2$, the terms of (5.1.2) vanish. Thus, the finite $\mathcal{O}$-module $\operatorname{Ext}_{\widetilde{U}_{\zeta}}^{n}\left(\widetilde{L}_{\zeta}(\lambda), \widetilde{\nabla}_{\zeta}(\mu)\right)$ is torsion-free (and possibly 0), so it is free. Therefore, (5.1.1) follows from (1.5.4). 
Corollary 5.2. Assume that $p>h$. For $\lambda \in X^{+}$, write $p \lambda=x \cdot \tau^{-}, x \in W_{p}$ and $\tau^{-} \in C_{\mathbb{Z}}^{-}$. Then $\Delta(\lambda)^{(1)}$ satisfies the $\mathrm{hLCF}$ condition, in the sense that

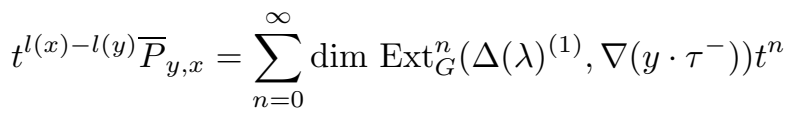

for any $y \in W_{p}$ such that $y \cdot \tau^{-} \in X^{+}$. In addition, we have

$$
\mu(y, x)=\operatorname{dim} \operatorname{Ext}_{G}^{1}\left(\Delta(\lambda)^{(1)}, \nabla\left(y \cdot \tau^{-}\right)\right) \leq 1,
$$

where $\mu(y, x)$ is the coefficient of $t^{l(x)-l(y)-1}$ in $P_{y, x}$ (cf. footnote 1$)$.

Proof. Clearly, $\widetilde{L}_{\zeta}^{\min }(p \lambda)$ satisfies $\widetilde{L}_{\zeta}^{\min }(p \lambda) / \pi \widetilde{L}_{\zeta}^{\min }(p \lambda) \cong \Delta(\lambda)^{(1)}$ (from the universal mapping property of $\Delta(\lambda)$ ). By Theorem 4.3, $\Delta(\lambda)^{(1)} \in \mathcal{E}^{L}$. The result (5.2.1) now follows as in the proof of the theorem.

Finally, observe that (5.2.1) implies (5.2.2), by Proposition4.2 and the fact that $\mathfrak{p}_{0}(\sigma)=\delta_{\sigma, 0}$ for $\sigma \in X$.

For $\lambda \in X_{\text {reg }}^{+}$, let $t=-1$ in (5.2.1). Then Lemma 1.1(b) gives that $\operatorname{ch} \Delta(\lambda)^{(1)}=$ $\chi(\lambda)^{(1)}=\chi_{\mathrm{KL}}(p \lambda)$, which is just the special $\lambda_{0}=0$ case of Lemma 1.3 Neither Lemma 1.3 nor Corollary 5.2 depend in any way on the assumption that the LCF holds in an ideal.

The above discussion provides some evidence for a potentially far-reaching question involving the category of rational $G$-modules. We begin with the following definition.

Definition 5.3. The left (resp., right) homological lattice property $\mathrm{hLP}^{L}$ (resp., $\mathrm{hLP}^{R}$ ) holds for $\lambda \in X_{\text {reg }}^{+}$provided that $L_{\zeta}(\lambda)$ has an admissible lattice $\widetilde{L}_{\zeta}(\lambda)$ (resp., $\left.\widetilde{L}_{\zeta}^{\prime}(\lambda)\right)$ such that $\operatorname{Ext}_{\widetilde{U}_{\zeta}}^{\bullet}\left(\widetilde{L}_{\zeta}(\lambda), \widetilde{\nabla}_{\zeta}(\mu)\right)$ (resp., $\left.\operatorname{Ext}_{\widetilde{U}_{\zeta}}^{\bullet}\left(\widetilde{\Delta}_{\zeta}(\mu), \widetilde{L}_{\zeta}^{\prime}(\lambda)\right)\right)$ is $\mathcal{O}$ torsion-free for any dominant weight $\mu \leq \lambda$.

The two lattices $\widetilde{L}_{\zeta}(\lambda)$ and $\widetilde{L}_{\zeta}^{\prime}(\lambda)$ appearing in the conditions $\mathrm{hLP}^{L}$ and $\mathrm{hLP}^{R}$ may not be the same. Equality does hold when and only when $\widetilde{L}_{\zeta}(\lambda) /$ $\pi \widetilde{L}_{\zeta}(\lambda)$ and $\widetilde{L}_{\zeta}^{\prime}(\lambda) / \pi \widetilde{L}_{\zeta}^{\prime}(\lambda)$ are irreducible (and hence isomorphic to $L(\lambda)$ ), as discussed in more detail below. This irreducibility condition holds, along with $\mathrm{hLP}^{L}$ and $\mathrm{hLP}^{R}$, when the LCF holds on $\Gamma \cap X_{\text {reg }}^{+}$for some finite ideal $\Gamma$.

The $n=0,1$ case of the $\mathrm{hLP}^{L}$ implies there is an exact sequence

$$
\begin{aligned}
0 & \rightarrow \operatorname{Hom}_{\widetilde{U}_{\zeta}}\left(\widetilde{L}_{\zeta}(\lambda), \widetilde{\nabla}_{\zeta}(\mu)\right) \stackrel{\pi}{\rightarrow} \operatorname{Hom}_{\widetilde{U}_{\zeta}}\left(\widetilde{L}_{\zeta}(\lambda), \widetilde{\nabla}_{\zeta}(\mu)\right) \\
& \rightarrow \operatorname{Hom}_{G}\left(\widetilde{L}_{\zeta}(\lambda) / \pi \widetilde{L}_{\zeta}(\lambda), \nabla(\mu)\right) \rightarrow 0
\end{aligned}
$$

similar to (5.1.2). Thus, $\widetilde{L}_{\zeta}(\lambda) / \pi \widetilde{L}_{\zeta}(\lambda)$ has simple head $L(\lambda)$. By Nakayama's lemma, $\widetilde{L}_{\zeta}(\lambda)$ is the lattice in $L_{\zeta}(\lambda)$ generated by some vector $v^{+} \in \widetilde{L}_{\zeta}(\lambda) \backslash \pi \widetilde{L}_{\zeta}(\lambda)$ of weight $\lambda$, i.e., $\widetilde{L}_{\zeta}(\lambda)=\widetilde{L}_{\zeta}^{\min }(\lambda)$.

Similarly, if the $\mathrm{hLP}^{R}$ holds for $\lambda$, then the required lattice $\widetilde{L}_{\zeta}(\lambda)$ is unique up to isomorphism, and can be taken to be $L_{\zeta}^{\max }(\lambda)$. 
Theorem 5.4. Assume that $p>h$.

(a) For $\lambda \in X_{\text {reg }}^{+}, \mathrm{hLP}^{L}$ (resp., $\left.\mathrm{hLP}^{R}\right)$ holds if and only if $\Delta^{\mathrm{red}}(\lambda)[-l(\lambda)] \in \mathcal{E}^{L}$ (resp., $\left.\nabla_{\text {red }}(\lambda)[-l(\lambda)] \in \mathcal{E}^{R}\right)$.

(b) Suppose that $\mathrm{hLP}^{L}$ (resp., $\mathrm{hLP}^{R}$ ) holds for $\lambda, \mu \in X_{\mathrm{reg}}^{+}$. Then

$$
\begin{aligned}
\operatorname{dim} \operatorname{Ext}_{G}^{n}\left(\Delta^{\operatorname{red}}(\lambda), \nabla_{\text {red }}(\mu)\right) & \\
& \left.=\sum_{m=0}^{n} \sum_{\nu} \operatorname{dim} \operatorname{Ext}_{G}^{m}\left(\Delta^{\text {red }}(\lambda), \nabla(\nu)\right) \cdot \operatorname{dim}_{\operatorname{Ext}_{G}^{n-m}}^{n-m}(\nu), \nabla_{\mathrm{red}}(\mu)\right) .
\end{aligned}
$$

Furthermore, if $\lambda=x \cdot \lambda^{-}$, where $\lambda^{-} \in C_{\mathbb{Z}}^{-}$, then

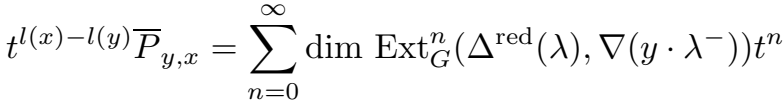

$$
\begin{aligned}
& =\sum_{n=0}^{\infty} \operatorname{dim} \operatorname{Ext}_{G}^{n}\left(\Delta\left(y \cdot \lambda^{-}\right), \nabla_{\text {red }}(\lambda)\right) t^{n} .
\end{aligned}
$$

In particular,

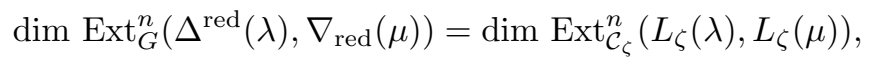

as given in (1.5.2).

Proof. We first prove (a). If $\Delta^{\text {red }}(\lambda)[-l(\lambda)] \in \mathcal{E}^{L}$, then, just as in the proof of Theorem 5.1, there is, for every integer $n$, a short exact sequence

$$
0 \rightarrow \operatorname{Ext}_{\tilde{U}_{\zeta}}^{n}\left(\widetilde{L}_{\zeta}(\lambda), \widetilde{\nabla}_{\zeta}(\mu)\right) \stackrel{\pi}{\rightarrow} \operatorname{Ext}_{\tilde{U}_{\zeta}}^{n}\left(\widetilde{L}_{\zeta}(\lambda), \widetilde{\nabla}_{\zeta}(\mu)\right) \rightarrow \operatorname{Ext}_{G}^{n}\left(\Delta^{\mathrm{red}}(\lambda), \nabla(\mu)\right) \rightarrow 0
$$

which implies that $\mathrm{hLP}^{L}$ holds for $\lambda$. Conversely, if $\mathrm{hLP}^{L}$ holds for $\lambda$, then we obtain the same short exact sequence, so that $L(\lambda)[-l(\lambda)] \in \mathcal{E}^{L}$, using (1.5.4) and Remark 1.5. A similar argument applies for the other half of (a). 1.5.

Finally, (b) follows immediately from (a) and [13, Thm. 3.5]. See also Remark

Remark 5.5. Suppose that $p>h$. Suppose $\lambda=x \cdot \lambda^{-}, \lambda^{-} \in C_{\mathbb{Z}}^{-}$, satisfies the $\mathrm{hLP}^{L}$ (resp., hLP ${ }^{R}$ ). Let $\mu=x \cdot \mu^{-}$for $\mu^{-} \in C_{\mathbb{Z}}^{-}$. Then $\mu$ satisfies the $\operatorname{hLP}^{L}$ (resp., $\left.\mathrm{hLP}^{R}\right)$.

To see this, consider the assertion for $\mathrm{hLP}^{L}$; the other case is similar. Let $\mathcal{B}$ (resp., $\mathcal{B}^{\prime}$ ) be the block (in the category of rational $G$-modules) determined by the linkage class $W_{p} \cdot \lambda^{-} \cap X^{+}$(resp., $W_{p} \cdot \mu^{-}$). Let $T=T_{\lambda}^{\mu}: \mathcal{B} \rightarrow \mathcal{B}^{\prime}$ be the highest weight category equivalence defined by translation. Then $T$ takes the full subcategory $\mathcal{E}^{L}(\mathcal{B})$ of $\mathcal{E}^{L}$ to the analogous full subcategory $\mathcal{E}^{L}\left(\mathcal{B}^{\prime}\right)$. The functor 
$T$ can be defined for the categories of integrable, type $1 U_{\zeta}$-modules [5, §8.3] and integrable, type $1 \widetilde{U}_{\zeta}$-modules 4 Furthermore, it commutes with base change $-\otimes_{\mathcal{O}} k$, so it carries $\Delta^{\text {red }}(x \cdot \lambda)$ to $\Delta^{\text {red }}(x \cdot \mu)$.

\section{Some General RESUlts AND CONJECtures}

We begin with some conjectures on the families $\left\{\Delta^{\text {red }}(\lambda)\right\}_{\lambda \in X_{\text {reg }}^{+}}$and $\left\{\nabla_{\text {red }}(\lambda)\right\}_{\lambda \in X_{\text {reg }}^{+}}$of indecomposable rational $G$-modules. We will see in Theorem 6.8 that the first three conjectures are theorems, if one assumes the validity of (a form of) the Lusztig conjecture in positive characteristic $p$. This latter conjecture is itself a theorem, though, presently, only for $p$ very large (the size unknown, depending on the root system) [4. Still, we are able to present some striking applications, handling smaller primes separately, in Section 7 . The remaining two conjectures we formulate below are proved in part under the same hypothesis (of a valid Lusztig conjecture) in Theorem 6.9.

Independently of the validity of these conjectures, the modules above have characters given by

$$
\operatorname{ch} \Delta^{\mathrm{red}}(\lambda)=\operatorname{ch} \nabla_{\mathrm{red}}(\lambda)=\sum_{y \cdot \lambda^{-} \in X^{+}}(-1)^{l(x)-l(y)} P_{y, x}(-1) \operatorname{ch} \Delta\left(y \cdot \lambda^{-}\right),
$$

where $\lambda=x \cdot \lambda^{-}$with $\lambda^{-} \in C^{-}$. (Recall the assumption in Remark 1.5.)

Conjecture 6.1. Assume that $p>h$. The left and right homological lattice prop-

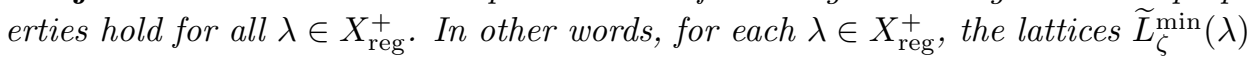
and $\widetilde{L}_{\zeta}^{\max }(\lambda)$ of $L_{\zeta}(\lambda)$ have the property that the $\mathcal{O}$-modules $\operatorname{Ext}_{\tilde{U}_{\zeta}}^{\bullet}\left(\widetilde{L}_{\zeta}^{\min }(\lambda), \widetilde{\nabla}_{\zeta}(\mu)\right)$ and $\operatorname{Ext}_{\widetilde{U}_{\zeta}}^{\bullet}\left(\widetilde{\Delta}(\lambda), \widetilde{L}_{\zeta}^{\max }(\lambda)\right)$ are torsion-free for all $\mu \in X_{\mathrm{reg}}^{+}$.

Conjecture 6.2. Assume that $p>h$. For each $\lambda \in X_{\text {reg }}^{+}, \Delta^{\text {red }}(\lambda)[-l(\lambda)] \in \mathcal{E}^{L}$ and $\nabla_{\text {red }}(\lambda)[-l(\lambda)] \in \mathcal{E}^{R}$. (Equivalently, for $\lambda \in X_{\text {reg }}^{+}, \Delta^{\text {red }}(\lambda) \in \widehat{\mathcal{E}^{L}}$ and $\nabla_{\text {red }}(\lambda) \in$ $\widehat{\mathcal{E}^{R}}$.)

The parenthetic comments follow from Lemma 2.1. Also, by Theorem 5.4, we have the following observation.

\footnotetext{
${ }^{4}$ The definition in 5 58.3] applies for this latter category, after some additional observations. In the definition [5] \$8.3], one tensors with a certain costandard module and then takes a projection onto a linkage class. The costandard module has an analogue "over $\mathcal{O}$ " (even over the algebra $\mathcal{A}$ ) in [5] which integrable, type 1 . Tensor products of integrable, type $1 \widetilde{U}_{\zeta^{-}}$-modules are (obviously) integrable, type 1 . Projection onto a linkage class makes sense in the category of integrable, type $1 \widetilde{U}_{\zeta}$-modules: First, one can talk about "composition factors" for $\widetilde{U}_{\zeta}$-modules and their weights. The category of all finite rank, integrable, type 1 modules is an $\mathcal{O}$-finite highest weight category $\widetilde{\mathcal{C}}$ in the sense of $[20, \S 2]$. The category $\widetilde{\mathcal{C}}[\Gamma]$ of all modules in $\widetilde{\mathcal{C}}$ whose composition factors have only high weights belonging to a fixed finite poset ideal $\Gamma$ in $X^{+}$has enough projective modules, and its projective indecomposable modules reduce modulo $p$ to projective indecomposable modules [20. $\S \S 2,3]$. Projections onto linkage classes can now be made in the category $\widetilde{\mathcal{C}}[\Gamma]$ for any sufficiently large $\Gamma$.
} 
Proposition 6.3. Conjecture $6.1 \Longleftrightarrow$ Conjecture 6.2 ,

There is a variation on Conjecture 6.2

Conjecture 6.4. Assume that $p>h$. For $\lambda \in X_{\text {reg }}^{+}$, write $\lambda=\lambda_{0}+p \lambda_{1}$, with $\lambda_{0} \in X_{1}^{+}$and $\lambda_{1} \in X^{+}$, and set

$$
\Delta^{\mathrm{red}}(\lambda)^{\prime}:=L\left(\lambda_{0}\right) \otimes \Delta\left(\lambda_{1}\right)^{(1)}, \quad \nabla_{\mathrm{red}}(\lambda)^{\prime}:=L\left(\lambda_{0}\right) \otimes \nabla\left(\lambda_{1}\right)^{(1)} .
$$

Then $\Delta^{\mathrm{red}}(\lambda)^{\prime}[-l(\lambda)] \in \mathcal{E}^{L}$ and $\nabla_{\mathrm{red}}(\lambda)^{\prime}[-l(\lambda)] \in \mathcal{E}^{R}$.

Conjecture 6.5. Assume that $p>h$. For $\lambda \in X^{+}, \Delta(\lambda)$ (resp., $\nabla(\lambda)$ ) has a $\Delta^{\text {red }}$-filtration, i.e., a filtration as a G-module with sections of the form $\Delta^{\text {red }}(\nu)$ (resp., $\left.\nabla_{\text {red }}(\nu)\right), \nu \in X^{+}$.

It seems likely that it is enough to check Conjecture 6.5 in the special case when $\lambda \in X_{\text {reg }}^{+}$where $\lambda \in W_{p} \cdot(-2 \rho) \cap X^{+}$, since the conclusion should behave well with respect to translation within an alcove or translation to a wall. Also, as far as we know, the assumption $p \geq h$ is sufficient in any of the above conjectures 5 We have the following variation of the last conjecture, using the modules introduced in Conjecture 6.4.

Conjecture 6.6. For $\lambda \in X^{+}, \Delta(\lambda)$ (resp., $\nabla(\lambda)$ ) has a $\Delta^{\text {red }^{\prime}}$-filtration (resp., a $\nabla_{\text {red }}^{\prime}$-filtration), i.e., a filtration as a $G$-module with sections of the form $\Delta^{\text {red }}(\nu)^{\prime}$ (resp., $\left.\nabla_{\text {red }}(\nu)^{\prime}\right), \nu \in X^{+}$.

Remark 6.7. In 2, a rational $G$-module $M$ is said to have a good $p$-filtration provided that it has a filtration with sections of the form $\nabla_{\text {red }}(\lambda)^{\prime}$. Then [2, Cor. 3.7] asserts that if $p \geq 2 h-2$, every $\nabla(\lambda)$ has a good $p$-filtration for each $\lambda \in X^{+}$. However, as pointed out to us by Andersen (in a private communication), the proof of [2, Lemma 3.3] does not hold, and so [2, Cor. 3.7] remains unproved.

Theorem 6.8. Let $p>h$.

(a) Assume that the LCF holds for all regular weights in $X_{1}^{+}$. Then Conjectures 6.1, 6.2 and 6.4 are true.

(b) If Conjecture 6.4 holds and $p \geq 2 h-2$, then the LCF holds for all regular weights in $X_{1}^{+}$.

Proof. First, we prove (a). By Proposition 1.7, the hypothesis implies that if $\lambda \in$ $X_{\text {reg }}^{+}$, then $\Delta^{\text {red }}(\lambda)=\Delta^{\text {red }}(\lambda)^{\prime} \cong L(\lambda)$ and $\nabla_{\text {red }}(\lambda)=\nabla_{\text {red }}(\lambda)^{\prime} \cong L(\lambda)$. Suppose that $\lambda, \lambda s \in X_{1}^{+}$are regular restricted weights and that $\lambda<\lambda s$, where $s \in S_{p}$. Suppose for $\omega \in X^{+}$that $\Delta^{\text {red }}(\lambda+p \omega)=L(\lambda) \otimes \Delta(\omega)^{(1)} \in \widehat{\mathcal{E}^{L}}$. Let the wall separating the dominant weights $\lambda+p \omega$ and $\lambda s+p \omega$ be of type $s^{\prime}$. By Corollary 3.3 and Lemma 3.4. $\beta_{s^{\prime}}$ is defined at $M:=L(\lambda) \otimes \Delta(\omega)^{(1)}$ and $\beta_{s^{\prime}} M \in \widehat{\mathcal{E}^{L}}$. It suffices to prove that $L(\lambda s) \otimes \Delta(\omega)^{(1)}$ is a direct summand of $\beta_{s^{\prime}}\left(L(\lambda) \otimes \Delta(\omega)^{(1)}\right)$. But in the category $G_{1} B-\bmod$,

$$
\widehat{\beta}_{s^{\prime}}\left(p \omega \otimes \widehat{L}_{1}(\lambda)\right) \cong p \omega \otimes \widehat{\beta}_{s} \widehat{L}_{1}(\lambda s),
$$

for some $s \in S_{p}$, using Lemma 3.1. The LCF holds for regular restricted weights so that $L(\lambda s)$ is a direct summand of $\beta_{s} L(\lambda)$. Also, $\left.\widehat{\beta}_{s} \widehat{L}_{1}(\lambda) \cong \beta_{s} L(\lambda)\right|_{G_{1} B}$. We

\footnotetext{
${ }^{5}$ But Conjecture 6.5 cannot be extended to all primes $p$. We are grateful to Will Turner for indicating that there is a type $A$ counterexample when $p=2$. We hope to discuss the example in a later paper.
} 
conclude that $p \omega \otimes \widehat{L}_{1}(\lambda s)$ is a direct summand of $\widehat{\beta}_{s^{\prime}}\left(p \omega \otimes \widehat{L}_{1}(\lambda)\right)$. Now apply the induction functor $\operatorname{ind}_{G_{1} B}^{G}$ and Lemma 3.1 to conclude that $L(\lambda s) \otimes \Delta(\omega)^{(1)}$ is a direct summand of $\beta_{s^{\prime}}\left(L(\lambda) \otimes \Delta(\omega)^{(1)}\right)$. By Theorem 4.3 for $\omega \in X^{+}, \Delta^{\text {red }}(p \omega) \cong$ $\Delta(\omega)^{(1)} \in \widehat{\mathcal{E}^{L}}$. Since any regular restricted weight can be connected to a weight in $C_{\mathbb{Z}}^{+}$by a series of adjacent restricted weights 6 we can now apply Remark 5.5 to conclude that (a) holds.

(b) Suppose that Conjecture 6.4 holds and that $p \geq 2 h-2$. Then if $\lambda \in X_{1}^{+}$, the well-known inequality $\left(\lambda+\rho, \alpha+0^{\vee}\right)<p(p-h+2)$ holds. Let $\mu \in X_{\text {reg }}^{+}$satisfy $\mu \leq \lambda$, and write $\mu=\mu_{0}+p \mu_{1}, \mu_{0} \in X_{1}^{+}$. Because $\left(\mu+\rho, \alpha_{0}^{\vee}\right) \leq\left(\lambda+\rho, \alpha_{0}^{\vee}\right)$, we see that $\left(\mu_{1}+\rho, \alpha_{0}^{\vee}\right) \leq p$, i.e., $\mu_{1} \in \overline{C_{\mathbb{Z}}^{+}}$. Thus, $L(\mu) \cong \Delta^{\text {red }}(\mu)^{\prime}$, so that $L(\mu)[-l(\mu)] \in \mathcal{E}^{L}$. Therefore, by Lemma 2.2 (taking $\Gamma$ to be the ideal in the poset $\left(X^{+}, \uparrow\right)$ of regular weight generated by the regular restricted weights), the LCF holds for $\lambda$.

For $\lambda \in X_{\text {reg }}^{+}$, put

$$
E_{\zeta}(\lambda)=\Delta_{\zeta}(\lambda) / \operatorname{rad}^{2} \Delta_{\zeta}(\lambda)
$$

Let $\widetilde{E}(\lambda)$ be the image of $\widetilde{\Delta}_{\zeta}(\lambda)$ in $E_{\zeta}(\lambda)$, and set $E(\lambda)=\widetilde{E}(\lambda) / \pi \widetilde{E}(\lambda)$. Observe that $\widetilde{E}(\lambda)$ is an admissible lattice for $E_{\zeta}(\lambda)$.

We are not yet able to establish Conjectures 6.4 and 6.5 under an LCF assumption like that given in Theorem 6.8(a). However, we have the following partial result.

Theorem 6.9. Assume that $p>h$ and that the LCF holds for all regular weights in $X_{1}^{+}$. With $\widetilde{E}(\lambda)$ as in (6.8.1), let $\widetilde{D}(\lambda)$ be the kernel of the natural surjection $\widetilde{E}(\lambda) \rightarrow \widetilde{L}^{\min }(\lambda)$, and similarly let $D_{\zeta}(\lambda)$ be the kernel of $E_{\zeta}(\lambda) \rightarrow L_{\zeta}(\lambda)$. (Thus, $\widetilde{D}(\lambda)$ is an admissible lattice for $D_{\zeta}(\lambda)$.) Then $\widetilde{D}(\lambda)$ has a $\widetilde{\mathcal{C}}_{\zeta}$-filtration with (distinct) sections $\widetilde{L}^{\min }(\mu)^{\oplus n_{\mu}}$, where $n_{\mu}=n_{\mu}(\lambda)=n_{\mu}=\left[D_{\zeta}(\lambda): L_{\zeta}(\mu)\right]=$ $\operatorname{dim} \operatorname{Ext}_{\mathcal{C}_{\zeta}}^{1}\left(L_{\zeta}(\lambda), L_{\zeta}(\mu)\right)$ for $\mu<\lambda$. In particular, $E(\lambda)$ has a $\Delta^{\text {red }}$-filtration with top section $\Delta^{\text {red }}(\lambda)$.

Proof. Let $\Gamma$ be any ideal of dominant weights with $\Gamma \subseteq(-\infty, \lambda)$. Let $\widetilde{D}_{\Gamma}(\lambda)$ be the largest $\mathcal{O}$-free quotient of $\widetilde{D}(\lambda)$ such that each composition factor of $\widetilde{D}_{\Gamma}(\lambda) \otimes_{\mathcal{O}} K=$ $D_{\Gamma, \zeta}(\lambda)$ has high weight in $\Gamma$. Also, let $\widetilde{E}_{\Gamma}(\lambda)$ be the resulting extension (obtained by forming the evident pushout diagram) of $\widetilde{L}^{\min }(\lambda)$ by $\widetilde{D}_{\Gamma}(\lambda)$. The conclusion of the theorem has an evident analogue with $\widetilde{E}(\lambda)$ replaced by $\widetilde{E}_{\Gamma}(\lambda), \widetilde{D}(\lambda)$ replaced by $\widetilde{D}_{\Gamma}(\lambda)$, and $\mu$ required to be in $\Gamma$.

We proceed by induction on $|\Gamma|$ starting with the case $\Gamma=\emptyset$. Thus, we assume that we are given $\Gamma$ so that $\widetilde{D}_{\Gamma}(\lambda)$ has a filtration with sections $\widetilde{L}^{\min }(\mu)^{\oplus n_{\mu}}$ for

\footnotetext{
${ }^{6}$ To see this fact, we argue as follows: Given a restricted regular weight $x$ in an alcove $C$, draw a (straight) line from $x$ to 0 . The line must pass through at least one reflecting affine hyperplane $H$, and we pick a point $z$ which is the nearest such intersection to $x$. Let $B$ be any open ball around $z$. Then $B$ contains points of $C$, and so the intersection of $C$ with $B$ is a nonempty open set. Also, the point $z$ is on the boundary of $C$, and, as such lies in the closure of a wall $F$ of $C$ whose containing hyperplane $H$ also contains $z$. The intersection of the ball $B$ with $F$ is nonempty, since $z \in \bar{F}$. Let $C^{\prime}$ be the unique alcove other than $C$ which has $F$ as a wall. Since any point of $F$ is the limit of points of $C^{\prime}$, the intersection of $B$ with $C^{\prime}$ is nonempty. Taking $B$ small enough, we may assume that $B$ is entirely contained in the open restricted parallelopiped. So $C^{\prime}$ must be restricted, and, not being on the same side of $H$ as $x$, is on the side of $H$ containing 0 , and is therefore "smaller". A reflection of $x$ into $C^{\prime}$ and induction gives the desired result.
} 
$\mu \in \Gamma$ and $n_{\mu}=\left[D_{\Gamma, \zeta}(\lambda): L_{\zeta}(\mu)\right]=\operatorname{dim} \operatorname{Ext}_{\mathcal{C}_{\zeta}}^{1}\left(L_{\zeta}(\lambda), L_{\zeta}(\mu)\right)$. (Note that $n_{\mu}=0$ for all $\mu \in \Gamma$ satisfying $l(\mu) \equiv l(\lambda) \bmod 2$.)

Claim 1. Let $E_{\Gamma}(\lambda)=\widetilde{E}_{\Gamma}(\lambda) / \pi \widetilde{E}_{\Gamma}(\lambda)$ and $E_{\Gamma, \zeta}(\lambda)=\widetilde{E}_{\Gamma}(\lambda) \otimes_{\mathcal{O}} K$. Suppose $\omega<\lambda$ has parity opposite to that of $\lambda$. Then $\operatorname{Ext}_{\widetilde{\mathcal{C}}_{\zeta}}^{1}\left(\widetilde{E}_{\Gamma}(\lambda), \widetilde{L}^{\max }(\omega)\right)$ is torsion-free. Also,

$$
\operatorname{Ext}_{\widetilde{\mathcal{C}}_{\zeta}}^{1}\left(\widetilde{E}_{\Gamma}(\lambda), \widetilde{L}^{\max }(\omega)\right) / \pi \operatorname{Ext}_{\widetilde{\mathcal{C}}_{\zeta}}^{1}\left(\widetilde{E}_{\Gamma}(\lambda), \widetilde{L}^{\max }(\omega)\right) \cong \operatorname{Ext}_{G}^{1}\left(E_{\Gamma}(\lambda), \nabla_{\operatorname{red}}(\omega)\right)
$$

To verify this assertion, put $D_{\Gamma}(\lambda)=\widetilde{D}_{\Gamma}(\lambda) / \pi \widetilde{D}_{\Gamma}(\lambda)$. Also, $D_{\Gamma}(\lambda)$ has a filtration with sections $\Delta^{\text {red }}(\mu)$, with $n_{\mu} \neq 0$ (which implies that $\mu$ has the same parity as $\omega)$. Thus, $\operatorname{Ext}_{G}^{1}\left(D_{\Gamma}(\lambda), \nabla_{\text {red }}(\omega)\right)=0$ by Theorem 6.8 . Therefore, the long exact sequence of $\operatorname{Ext}_{G}^{\bullet}$ applied to the short exact sequence $0 \rightarrow D_{\Gamma}(\lambda) \rightarrow E_{\Gamma}(\lambda) \rightarrow$ $\Delta^{\text {red }}(\lambda) \rightarrow 0$ gives an exact sequence

$$
\begin{array}{r}
0 \rightarrow \operatorname{Hom}_{G}\left(D_{\Gamma}(\lambda), \nabla_{\text {red }}(\omega)\right) \rightarrow \operatorname{Ext}_{G}^{1}\left(\Delta^{\text {red }}(\lambda), \nabla_{\text {red }}(\omega)\right) \\
\rightarrow \operatorname{Ext}_{G}^{1}\left(E_{\Gamma}(\lambda), \nabla_{\text {red }}(\omega)\right) \rightarrow 0 .
\end{array}
$$

Next, $\operatorname{Ext}_{\mathcal{C}_{\zeta}}^{1}\left(D_{\Gamma, \zeta}(\lambda), L_{\zeta}(\omega)\right)=0$ by the quantum analogue of Lemma 2.2, since $D_{\Gamma, \zeta}(\lambda)$ is a direct sum of irreducible modules $L_{\zeta}(\mu)$ with $\mu$ having the same parity as $\omega$. Therefore, the short exact sequence $0 \rightarrow D_{\Gamma, \zeta}(\lambda) \rightarrow E_{\Gamma, \zeta}(\lambda) \rightarrow L_{\zeta}(\lambda) \rightarrow 0$ gives an exact sequence

$$
\begin{aligned}
0 \rightarrow \operatorname{Hom}_{\mathcal{C}_{\zeta}}\left(D_{\Gamma, \zeta}(\lambda), L_{\zeta}(\omega)\right) \operatorname{Ext}_{\mathcal{C}_{\zeta}}^{1}\left(L_{\zeta}(\lambda), L_{\zeta}(\omega)\right) & \\
& \rightarrow \operatorname{Ext}_{\mathcal{C}_{\zeta}}^{1}\left(E_{\Gamma, \zeta}(\lambda), L_{\zeta}(\omega)\right) \rightarrow 0 .
\end{aligned}
$$

Clearly, the left-hand Hom in (6.9.2) has dimension equal to the multiplicity of $L_{\zeta}(\omega)$ in $D_{\Gamma, \zeta}(\lambda)$. This multiplicity equals the multiplicity of $\Delta^{\text {red }}(\omega)$ as a section

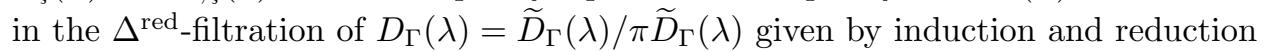
$\bmod \pi$. (Recall $\Delta^{\text {red }}(\omega)=\widetilde{L}^{\text {min }}(\omega) / \pi \widetilde{L}^{\text {min }}(\omega)$, and the module $\widetilde{L}^{\text {min }}(\omega)$ is torsionfree.) If $\Delta^{\text {red }}(\tau)$ is a section of this filtration, then $\tau$ and $\omega$ have the same parity, so, $\operatorname{Ext}_{G}^{1}\left(D_{\Gamma}(\lambda), \nabla_{\text {red }}(\omega)\right)=0$. Thus, $\operatorname{dim} \operatorname{Hom}_{G}\left(D_{\Gamma}(\lambda), \nabla_{\text {red }}(\omega)\right)$ also equals this multiplicity. It follows that

$$
\operatorname{dim} \operatorname{Hom}_{G}\left(D_{\Gamma}(\lambda), \nabla_{\text {red }}(\omega)\right)=\operatorname{dim} \operatorname{Hom}_{\mathcal{C}_{\zeta}}\left(D_{\Gamma, \zeta}(\lambda), L_{\zeta}(\lambda)\right) .
$$

By Theorem 5.4(b) and Theorem 6.8, the middle Ext ${ }^{1}$-terms in (6.9.1) and (6.9.2) also have the same dimension. Thus, the right-hand Ext ${ }^{1}$-terms have the same dimension, as well. Form the exact sequence

$$
\operatorname{Ext}_{\mathcal{C}_{\zeta}}^{1}\left(\widetilde{E}_{\Gamma}(\lambda), \widetilde{L}^{\max }(\omega)\right) \stackrel{\pi}{\rightarrow} \operatorname{Ext}_{\mathcal{C}_{\zeta}}^{1}\left(\widetilde{E}_{\Gamma}(\lambda), \widetilde{L}^{\max }(\omega)\right) \rightarrow \operatorname{Ext}_{\mathcal{C}_{\zeta}}^{1}\left(\widetilde{E}_{\Gamma}(\lambda), \nabla_{\text {red }}(\omega)\right)
$$

By (1.5.5), $\operatorname{Ext}_{\mathcal{C}_{\zeta}}^{1}\left(\widetilde{E}_{\Gamma}(\lambda), \nabla_{\text {red }}(\omega)\right) \cong \operatorname{Ext}_{G}^{1}\left(E_{\Gamma}(\lambda), \nabla_{\text {red }}(\omega)\right)$, so that Claim 1 now follows.

Claim 2. If $\omega<\lambda \operatorname{Ext}_{\mathcal{C}_{\zeta}}^{1}\left(L_{\zeta}(\lambda), L_{\zeta}(\omega)\right) \neq 0$ if and only if $\left[D_{\zeta}(\lambda): L_{\zeta}(\omega)\right] \neq 0$.

Claim 3. Assume that $\left[D_{\zeta}(\lambda): L_{\zeta}(\omega)\right] \neq 0$ (so that $\omega<\lambda$, and $\omega$ and $\lambda$ have opposite parity). Then $\omega \in \Gamma \Longleftrightarrow \operatorname{Ext}_{G}^{1}\left(E_{\Gamma}(\lambda), \nabla_{\text {red }}(\omega)\right)=0$.

Claim 2 is clear, so consider Claim 3. First, assume that $\omega \in \Gamma$. Then in (6.9.2), the inclusion $\iota: \operatorname{Hom}_{\mathcal{C}_{\zeta}}\left(D_{\Gamma, \zeta}(\lambda), L_{\zeta}(\omega)\right) \hookrightarrow \operatorname{Ext}_{\mathcal{C}_{\zeta}}^{1}\left(L_{\zeta}(\lambda), L_{\zeta}(\omega)\right)$ is an isomorphism, so that $\operatorname{Ext}_{G}^{1}\left(E_{\Gamma}(\lambda), \nabla_{\text {red }}(\omega)\right)=0$ by (6.9.2) and Claim 1. Conversely, if $\operatorname{Ext}_{G}^{1}\left(E_{\Gamma}(\lambda), \nabla_{\text {red }}(\omega)\right)=0$, then Claim 1 implies that $\iota$ is an isomorphism so that $\omega \in \Gamma$. This proves Claim 3 . 
Claim 4. If $\omega \in \Gamma$ has parity opposite to $\lambda$, then $\operatorname{Ext}_{G}^{1}\left(E_{\Gamma}(\lambda), L(\omega)\right)=0$.

Since $\operatorname{Hom}_{G}\left(E_{\Gamma}(\lambda), \nabla_{\text {red }}(\omega) / L(\omega)\right)=0$, the claim follows from the long exact sequence of $\operatorname{Ext}_{G}^{\bullet}$ provided that we know that

$$
\operatorname{Ext}_{G}^{1}\left(E_{\Gamma}(\lambda), \nabla_{\text {red }}(\omega)\right)=0 .
$$

This later vanishing is true by Claim 3 , if $\left[D_{\zeta}(\lambda): L_{\zeta}(\omega)\right] \neq 0$. Otherwise, we have that $\operatorname{Ext}_{\mathcal{C}_{\zeta}}^{1}\left(L_{\zeta}(\lambda), L_{\zeta}(\omega)\right)=0$ by Claim 2, so the right-hand term of (6.9.2) vanishes. Thus, (6.9.4) holds by (1.5.4) and Claim 1. This proves Claim 4.

Claim 5. Let $\nu<\lambda$ of opposite parity to $\lambda$ with $\nu \notin \Gamma$. We have $\operatorname{Ext}_{G}^{1}\left(E_{\Gamma}(\lambda), \nabla_{\text {red }}(\nu)\right)$ $\cong \operatorname{Ext}_{G}^{1}\left(\Delta^{\text {red }}(\lambda), \nabla_{\text {red }}(\nu)\right)$, which has dimension equal to $\operatorname{dim} \operatorname{Ext}_{\mathcal{C}_{\zeta}}^{1}\left(L_{\zeta}(\lambda), L_{\zeta}(\nu)\right)$.

To prove the isomorphism, we use the fact that $D_{\Gamma}(\lambda)$ has, as previously noted, a filtration with sections $\Delta^{\text {red }}(\mu)$. A module $\Delta^{\text {red }}(\mu)$ appears as a section only when $\operatorname{Ext}_{\mathcal{C}_{\zeta}}^{1}\left(L_{\zeta}(\lambda), L_{\zeta}(\mu)\right) \neq 0$, which implies that $\mu, \nu$ have the same parity. Therefore, $\operatorname{Ext}_{G}^{1}\left(\Delta^{\mathrm{red}}(\mu), \nabla_{\text {red }}(\nu)\right)=0$, and so $\operatorname{Ext}_{G}^{1}\left(D_{\Gamma}(\lambda), \nabla_{\text {red }}(\nu)\right)=0$. Also, since $\nu \notin \Gamma$, we have $\operatorname{Hom}_{G}\left(D_{\Gamma}(\lambda), \nabla_{\text {red }}(\nu)\right)=0$. The first equality follows from the long exact sequence of $\operatorname{Ext}_{G}^{\bullet}$ applied to the short exact sequence $0 \rightarrow D_{\Gamma}(\lambda) \rightarrow E_{\Gamma}(\lambda) \rightarrow$ $\Delta^{\text {red }}(\lambda) \rightarrow 0$. The second equality is a consequence of Theorem [5.4(b). This proves Claim 5 .

We note also the obvious equality

$$
\operatorname{dim} \operatorname{Ext}_{\mathcal{C}_{\zeta}}^{1}\left(L_{\zeta}(\lambda), L_{\zeta}(\nu)\right)=\left[D_{\zeta}(\lambda): L_{\zeta}(\nu)\right]
$$

for any $\nu<\lambda$.

Assume that $\widetilde{D}_{\Gamma}(\lambda) \neq \widetilde{D}(\lambda)$. Let $\nu \in X^{+}$be minimal satisfying $\left[D_{\zeta}(\lambda)\right.$ : $\left.L_{\zeta}(\nu)\right] \neq 0$ and $\nu \notin \Gamma$. Necessarily, $\nu<\lambda$, and $\nu$ and $\lambda$ have opposite parity. Without loss, we can replace $\Gamma$ by $\Gamma^{\natural}=\left\{\omega \in X^{+} \mid \omega<\nu\right\}$ and keep $E_{\Gamma, \zeta}(\lambda)$, $D_{\Gamma, \zeta}(\lambda), \widetilde{D}_{\Gamma}(\lambda)$ the same. If $\Gamma \neq \Gamma^{\natural}$, we could enlarge $\Gamma$ to $\Gamma^{\natural}$. Thus, we may assume that $\Gamma=\Gamma^{\natural}$.

All composition factors of $\nabla_{\text {red }}(\nu)=\nabla\left(\nu_{1}\right)^{(1)} \otimes L\left(\nu_{0}\right)$ have high weight $\omega$ with the same parity as $\nu$, which is opposite to the parity of $\lambda$. Except for $\nu$, these weights $\omega$ also belong to $\Gamma$, by the minimality of $\nu$. By Claim 4,

$$
\operatorname{Ext}_{G}^{1}\left(E_{\Gamma}(\lambda), L(\omega)\right)=0
$$

for such $\omega$. In particular,

$$
\operatorname{Ext}_{G}^{1}\left(E_{\Gamma}(\lambda), L(\nu)\right) \cong \operatorname{Ext}_{G}^{1}\left(E_{\Gamma}(\lambda), \nabla_{\text {red }}(\nu)\right)
$$

by Claim 4 and the long exact sequence of cohomology. Also, there is an injection

$$
\operatorname{Ext}_{G}^{1}\left(E_{\Gamma}(\lambda), \Delta^{\mathrm{red}}(\nu)\right) \hookrightarrow \operatorname{Ext}_{G}^{1}\left(E_{\Gamma}(\lambda), L(\nu)\right) .
$$

The lattice inclusion $\widetilde{L}^{\min }(\nu) \subseteq \widetilde{L}^{\max }(\nu)$ has quotient which has a finite length with composition factors of the form $L(\omega), \omega<\nu$, where $L(\omega)$ is a composition factor of $\nabla_{\text {red }}(\nu)$. Thus, using (6.9.6) and (1.5.5), there is an identification

$$
\operatorname{Ext}_{\widetilde{\mathcal{C}}_{\zeta}}^{1}\left(\widetilde{E}_{\Gamma}(\lambda), \widetilde{L}^{\min }(\nu)\right) \cong \operatorname{Ext}_{\widetilde{\mathcal{C}}_{\zeta}}^{1}\left(\widetilde{E}_{\Gamma}(\lambda), \widetilde{L}^{\max }(\nu)\right) \text {. }
$$

By Claim 1, both sides of (6.9.9) are torsion-free. So the reduction modulo $\pi$ of the left-hand side injects to $\operatorname{Ext}_{G}^{1}\left(E_{\Gamma}(\lambda), \Delta^{\text {red }}(\nu)\right)$, which injects into $\operatorname{Ext}_{G}^{1}\left(E_{\Gamma}(\lambda), L(\nu)\right)$. The latter group is isomorphic to $\operatorname{Ext}_{G}^{1}\left(E_{\Gamma}(\lambda), \nabla_{\text {red }}(\nu)\right)$ by (6.9.7). By Claim 5, 
$\operatorname{dim} \operatorname{Ext}_{G}^{1}\left(E_{\Gamma}(\lambda), \nabla_{\text {red }}(\nu)\right)=\left[D_{\zeta}(\lambda): L_{\zeta}(\mu)\right]$, which is the rank of the right-hand side of (6.9.9). Thus,

$$
\operatorname{Ext}_{G}^{1}\left(E_{\Gamma}(\lambda), \Delta^{\mathrm{red}}(\nu)\right) \cong \operatorname{Ext}_{G}^{1}\left(E_{\Gamma}(\lambda), L(\nu)\right) \cong \operatorname{Ext}_{G}^{1}\left(E_{\Gamma}(\lambda), \nabla_{\mathrm{red}}(\nu)\right) .
$$

Also,

$$
\operatorname{Ext}_{G}^{1}\left(E_{\Gamma}(\lambda), \Delta^{\mathrm{red}}(\nu)\right) \cong \operatorname{Ext}_{\widetilde{\mathcal{C}}_{\zeta}}^{1}\left(\widetilde{E}_{\Gamma}(\lambda), \widetilde{L}^{\min }(\mu)\right) / \pi \operatorname{Ext}_{\widetilde{\mathcal{C}}_{\zeta}}^{1}\left(\widetilde{E}_{\Gamma}(\lambda), \widetilde{L}^{\min }(\mu)\right)
$$

and

$$
\operatorname{Ext}_{\widetilde{\mathcal{C}}_{\zeta}}^{1}\left(\widetilde{E}_{\Gamma}(\lambda), \widetilde{L}^{\min }(\mu)\right) \text { is a free } \mathcal{O} \text {-module of rank } n_{\mu} \text {. }
$$

To complete the proof of the "inductive step," put $\Gamma^{\prime}=\{\nu\} \cup \Gamma$. Form an extension

$$
0 \rightarrow \widetilde{L}^{\min }(\nu)^{\oplus n_{\nu}} \rightarrow \widetilde{E} \rightarrow \widetilde{E}_{\Gamma}(\lambda) \rightarrow 0
$$

using a $\mathcal{O}$-basis of $\operatorname{Ext}_{\widetilde{\mathcal{C}}_{\zeta}}^{1}\left(\widetilde{E}_{\Gamma}(\lambda), \widetilde{L}^{\mathrm{min}}(\nu)\right)$. By (6.9.10), 6.9.11), and (6.9.12), $\widetilde{E}$ has the same head $L(\lambda)$ as $\widetilde{E}_{\Gamma}(\lambda)$. (One gets the same head after reduction $\bmod \pi$, and even after factoring out by the radical of $\Delta^{\mathrm{red}}(\nu)^{\oplus n_{\nu}}$.) Thus, there is a surjection $\widetilde{\Delta}_{\zeta}(\lambda) \rightarrow \widetilde{E}$, which induces a surjection $\widetilde{E}(\lambda) \rightarrow \widetilde{E}$. The image $\widetilde{D}$ of $\widetilde{D}(\lambda)$ under this latter surjection is $\mathcal{O}$-free, and $\left[D_{\zeta}: L_{\zeta}(\omega)\right]=\left[D_{\zeta}(\lambda): L_{\zeta}(\omega)\right]=n_{\omega}$ for all $\omega \in \Gamma^{\prime}$, where $D_{\zeta}=\widetilde{D} \otimes_{\mathcal{O}} K$. Thus, $\widetilde{D}=\widetilde{D}_{\Gamma^{\prime}}(\lambda)$ and $\widetilde{E}=\widetilde{E}_{\Gamma^{\prime}}(\lambda)$.

We must check that $\widetilde{D}_{\Gamma^{\prime}}(\lambda)$ has a $\widetilde{\mathcal{C}}_{\zeta^{-}}$filtration with sections $\widetilde{L}^{\min }(\mu)^{\oplus n_{\mu}}$, where $\mu \in \Gamma^{\prime}, n_{\mu}=\left[D_{\zeta}(\lambda): L_{\zeta}(\mu)\right]=\operatorname{dim}_{\operatorname{Ext}_{\mathcal{C}_{\zeta}}^{1}}\left(L_{\zeta}(\lambda), L_{\zeta}(\mu)\right)$. This condition follows from (6.9.13), induction, and the calculation $\left[D_{\zeta}: L_{\zeta}(\omega)\right]=n_{\omega}$ above, with $\omega=$ $\mu$.

Remark 6.10. Let $D(\lambda)=\widetilde{D}(\lambda) / \pi \widetilde{D}(\lambda)$. The proof of Theorem 6.8 shows that

$$
\operatorname{Ext}_{G}^{1}\left(D(\lambda), \nabla_{\text {red }}(\omega)\right)=0
$$

for all $\omega \in X_{\text {reg }}^{+}$satisfying $\omega<\lambda$ and $l(\omega) \not \equiv l(\lambda) \bmod 2$. In a later paper, we will show that (assuming the LCF holds for restricted regular weights) the category of all rational $G$-modules with composition factors having regular dominant weights of a fixed parity forms a highest weight category whose standard and costandard modules are $\Delta^{\text {red }}(\mu)$ and $\nabla_{\text {red }}(\mu)$, respectively. Since all composition factors of $D(\lambda)$ are regular and have parity opposite to $\lambda$ and are smaller than $\lambda$, the above vanishing (6.10.1) result is precisely the standard criterion that $D(\lambda)$ (or $\widetilde{D}(\lambda)$ ) has a $\Delta^{\text {red }}$ ( or $\widetilde{L}^{\text {min }}$-)filtration in the highest weight category. This remark helps provide some conceptual insight into the above proof.

One can conjecturally extend the main conclusion of Theorem 6.9 to the entire radical series of $\Delta_{\zeta}(\lambda)$. For $\lambda \in X_{\text {reg }}^{+}$and $n \geq r$, put $E_{\zeta}^{n}(\lambda):=\Delta_{\zeta}(\lambda) / \operatorname{rad}^{n} \Delta_{\zeta}(\lambda)$, and let $\widetilde{E}^{n}(\lambda)$ be the image of $\widetilde{L}^{\min }(\lambda)$ in $E_{\zeta}^{n}(\lambda)$.

Conjecture 6.11. Assume that $p>h$ and let $\lambda \in X_{\mathrm{reg}}^{+}$. Let $\widetilde{D}^{n-1}(\lambda)$ be the kernel of the natural surjection $\widetilde{E}^{n}(\lambda) \rightarrow \widetilde{E}^{n-1}(\lambda)(n \geq 2)$. Then $\widetilde{D}^{n-1}(\lambda)$ has a filtration with sections $\widetilde{L}^{\min }(\mu), \mu \in X_{\text {reg. }}^{+}$. 


\section{Applichtions to Degree one cohomology}

Throughout this section, $G$ denotes a connected, simply connected, semisimple group, defined and split over $\mathbb{F}_{p}$. We let $\Phi$ be its root system. We apply the results of the previous section to obtain new results on the bounds of 1-cohomology for finite groups $G(q)$ of Lie type. We also obtain several results on $\operatorname{Ext}_{G}^{1}\left(L, L^{\prime}\right)$ and $H^{1}(G, L)$ for the algebraic group $G$ and irreducible modules $L, L^{\prime}$, relating these groups to quantum analogues. Given $\lambda \in X^{+}$, write $\lambda=\sum_{i=0}^{\infty} p^{i} \lambda_{i}$, where $\lambda_{i} \in X_{1}^{+}$. We make no assumption on $p$, except those explicitly noted below. Put

$$
\lambda^{(i)}=\sum_{j=i}^{\infty} p^{j-i} \lambda_{j} .
$$

By [1], $\operatorname{Ext}_{G_{1}}^{1}(\widehat{L}, \widehat{L}) \neq 0$ for some irreducible $G_{1}$-module $\widehat{L}$ if and only if $p=2$ and $\Phi$ has a component of type $C_{n}(n \geq 1)$. This statement holds if $\widehat{L}$ is replaced by an irreducible $G$-module $L(\lambda)$. When $\Phi$ is irreducible of type $C_{n}$ and $p=2$, we have $\operatorname{Ext}_{G_{1}}^{1}(L(0), L(0))^{(-1)} \cong \nabla\left(\varpi_{1}\right) \cong L\left(\varpi_{1}\right)$, the irreducible $2 n$-dimensional (standard) module of high weight $\varpi_{1}$; see, e.g., [26, 12.2]. On the other hand, in all cases, if $L$ is irreducible, then $\operatorname{Ext}_{G}^{1}(L, L)=0$.

The following result (at least part (a)) is essentially contained in [1]. Part (a) is a step in the proof of [1, Theorem 5.6], but is a step not requiring the hypothesis $p \geq 3 h-3$ of that theorem.

Lemma 7.1. Let $\lambda, \mu \in X^{+}$, and let $j$ be minimal with $\lambda_{j} \neq \mu_{j}$. (If $\lambda=\mu$, put $j=\infty$ and $\lambda^{(j)}=0$.)

(a) We have

$$
\operatorname{Ext}_{G}^{1}(L(\lambda), L(\mu)) \cong \operatorname{Ext}_{G}^{1}\left(L\left(\lambda^{(j)}\right), L\left(\mu^{(j)}\right)\right),
$$

unless $p=2$ and $\Phi$ has a component of type $C_{n}(n \geq 1)$.

(b) Assume that $\Phi$ is irreducible of type $C_{n}$ and that $p=2$, and let $\lambda=0$. Then (7.1.1) fails if and only if $\mu=2 \varpi_{1}$. In this case, we have $j=1$ and $\operatorname{Ext}_{G}^{1}(L(\lambda), L(\mu)) \cong H^{1}(G, L(\mu)) \cong k$, while $\operatorname{Ext}_{G}^{1}\left(L\left(\lambda^{(1)}\right), L\left(\mu^{(1)}\right)\right) \cong H^{1}\left(G, L\left(\varpi_{1}\right)\right)$ $=0$.

Proof. We prove (a). We can assume $\Phi$ is irreducible. Since $\operatorname{Ext}_{G}^{1}(L(\lambda), L(\lambda))=0$, we can assume that $\lambda \neq \mu$ and $j<\infty$. It suffices to show that if $\lambda_{0}=\mu_{0}$, then, $\operatorname{Ext}_{G}^{1}(L(\lambda), L(\mu)) \cong \operatorname{Ext}_{G}^{1}\left(L\left(\lambda^{(1)}\right), L\left(\mu^{(1)}\right)\right)$. In the Hochschild-Serre spectral sequence

$$
E_{2}^{s, t}=\operatorname{Ext}_{G}^{s}\left(L\left(\lambda^{(1)}\right), \operatorname{Ext}_{G_{1}}^{t}\left(L\left(\lambda_{0}\right), L\left(\lambda_{0}\right)\right)^{(-1)} \otimes L\left(\mu^{(1)}\right)\right) \Rightarrow \operatorname{Ext}_{G}^{s+t}(L(\lambda), L(\mu)),
$$

the $(s, t)=(0,1)$-term on the left is zero, unless $p=2$ and $\Phi$ has type $C_{n}$, as remarked above. Otherwise, $\operatorname{Ext}_{G_{1}}^{1}\left(L\left(\lambda_{0}\right), L\left(\lambda_{0}\right)\right)=0$ and equation (7.1.1) follows. This proves (a).

Now assume the hypothesis of (b). First, assume that (7.1.1) fails. By remarks before the statement of the lemma, we must have

$$
\begin{aligned}
E_{2}^{0,1} & =\operatorname{Hom}_{G}\left(L(0), \operatorname{Ext}_{G_{1}}^{1}(L(0), L(0))^{(-1)} \otimes L\left(\mu^{(1)}\right)\right) \\
& \cong \operatorname{Hom}_{G}\left(L\left(\mu^{(1)}\right), \operatorname{Ext}_{G_{1}}^{1}(L(0), L(0))^{(-1)}\right) \neq 0 .
\end{aligned}
$$

It follows that $\mu^{(1)}=\varpi_{1}$ (so $\left.\mu=p \varpi_{1}\right)$, since $\operatorname{Ext}_{G_{1}}^{1}(L(0), L(0))^{(-1)} \cong L\left(\varpi_{1}\right)$. Also, $E_{2}^{1,0} \cong E_{2}^{2,0}=0$ because $L\left(\varpi_{1}\right) \cong \nabla\left(\varpi_{1}\right)$ and $H^{\bullet}\left(G, \nabla\left(\varpi_{1}\right)\right)=0$. Thus, 
$\operatorname{Ext}_{G}^{1}(L(\lambda), L(\mu)) \cong \operatorname{Ext}_{G}^{1}(L(0), L(\mu)) \cong E_{2}^{0,1} \cong k$, as required. The converse statement follows similarly.

In the following result, the requirement $p>h$ is needed for Theorem 6.8. Also, the assumption $p>h$ is required to assume that the $L C F_{\zeta}$ holds for $U_{\zeta}$; cf. Remark 1.5 .

Theorem 7.2. Assume that $p>h$ and that the LCF holds for all regular weights in $X_{1}^{+}$. Let $\lambda, \mu \in X^{+}$be distinct weights with $\lambda>\mu$ and let $j$ be minimal with $\lambda_{j} \neq \mu_{j}$. Suppose that $\lambda^{(j)} \in X_{\mathrm{reg}}^{+}$. Then 7

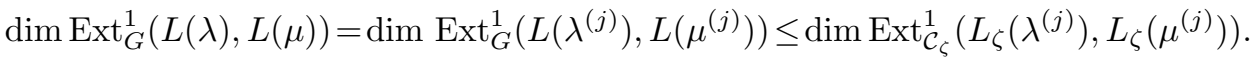

Proof. Using Lemma 7.1, we can assume that the restricted weights $\lambda_{0}, \mu_{0}$ are distinct and regular. The hypothesis and Proposition 1.7 imply that $\Delta^{\mathrm{red}}(\lambda) \cong L\left(\lambda_{0}\right) \otimes$ $\Delta\left(\lambda^{(1)}\right)^{(1)}$. Therefore, the composition factors of $\Delta^{\text {red }}(\lambda)$ have the form $L\left(\lambda_{0}\right) \otimes$ $L(\tau)^{(1)}$, where $\tau \in X^{+}$satisfies $\tau \leq \lambda^{(1)}$. Hence, Hom $\operatorname{Hom}_{G}\left(\operatorname{rad} \Delta^{\mathrm{red}}(\lambda), L(\mu)\right)=0$, so, by the long exact sequence of cohomology, we have an injection $\operatorname{Ext}_{G}^{1}(L(\lambda), L(\mu)) \hookrightarrow$ $\operatorname{Ext}_{G}\left(\Delta^{\text {red }}(\lambda), L(\mu)\right)$. Also, the inclusion $L(\mu) \hookrightarrow \nabla_{\text {red }}(\mu)$ induces an inclusion $\operatorname{Ext}_{G}^{1}\left(\Delta^{\text {red }}(\lambda), L(\mu)\right) \hookrightarrow \operatorname{Ext}_{G}^{1}\left(\Delta^{\text {red }}(\lambda), \nabla_{\text {red }}(\mu)\right)$ since there is no nonzero morphism $\Delta^{\mathrm{red}}(\lambda) \rightarrow \nabla_{\mathrm{red}}(\mu) / L(\mu)$ because $\lambda>\mu$. Therefore, composing these inclusions gives an inclusion $\operatorname{Ext}_{G}^{1}(L(\lambda), L(\mu)) \hookrightarrow \operatorname{Ext}_{G}^{1}\left(\Delta^{\text {red }}(\lambda), \nabla_{\text {red }}(\mu)\right)$. By Theorem 6.8.

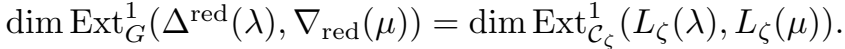

We will show later in Lemma 7.6 that $\operatorname{dim} \operatorname{Ext}_{\mathcal{C}_{\zeta}}^{1}\left(L_{\zeta}(\lambda), L_{\zeta}(\mu)\right)$ is bounded by a constant $\widehat{C}(\Phi)$ depending only on $\Phi$, for all $\lambda \in X_{\text {reg }}^{+}$and $\mu \in X^{+}$. Assuming $p>h$ ( $p \geq h$ is sufficient, if we know $L C F_{\zeta}$ hold $\&$ ), and requiring that $\lambda$ be regular, we may use, in place of $\widehat{C}(\Phi)$, a constant bound $E=E(\Phi)$ defined in terms of Kazhdan-Lusztig polynomials, namely

$$
E(\Phi)=\max \mu(y, w),
$$

where the max ranges over all $y, w \in W_{p}$ with $l(y)=l\left(w_{0}\right)+l\left(w_{0} y\right), l(w)=l\left(w_{0}\right)+$ $l\left(w_{0} w\right)$. (See (1.5.3).) Lemma 7.6 shows that the number $E(\Phi)$ is finite 9 The precise value of $E(\Phi)$ is unknown, as is the value of a related constant $E_{0}(\Phi)$, which we define as

$$
E_{0}(\Phi)=\max \mu\left(w_{0}, w\right),
$$

where max ranges over all $w \in W_{p}$ with $l(w)=l\left(w_{0}\right)+l\left(w_{0} w\right)$. That is, $E_{0}(\Phi)$ is defined like $E(\Phi)$, except with $y$ fixed, as $y=w_{0}$. Thus, $y \cdot(-2 \rho)=0$, and $E_{0}(\Phi)$ is a bound, for $p>h$, on $\operatorname{dim}_{\operatorname{Ext}_{\mathcal{C}_{\zeta}}^{1}}^{1}\left(L_{\zeta}(\lambda), L_{\zeta}(\mu)\right)$ when $\lambda=0$, the 1-cohomology case.

Theorem 7.3. There is a constant $C=C(\Phi)$, depending only on $\Phi$, such that $\operatorname{dim} H^{1}(G, L(\mu)) \leq C, \forall \mu \in X^{+}$. Also, if we consider only characteristics $p>h$

\footnotetext{
${ }^{7}$ It is quite possible that this restriction of regularity can be removed, at least in many cases. For results and conjectures comparing Ext $^{1}$ between irreducible modules with singular high weights and $\mathrm{Ext}^{1}$ for irreducible modules with related regular high weights, see [40. §4].

${ }^{8}$ Recall that $p \neq 2$ always and $p>3$ if $\Phi$ has a component of type $G_{2}$.

${ }^{9} \mathrm{~A}$ combinatorial proof of this fact can be found in [41, Thm. 3.1], which proves the equivalent fact that the numbers $\mu(y, w)$ are bounded in the lowest 2-sided cell in $W_{p}$. Also, [41, Thm. 3.3] shows that, in type $\widetilde{A}_{n}, E(\Phi) \rightarrow \infty$ as $n \rightarrow \infty$.
} 
for which the $L C F$ holds for all regular weights in $X_{1}^{+}$, then $\operatorname{dim} H^{1}(G, L(\mu)) \leq$ $E_{0}(\Phi), \forall \mu \in X^{+}$.

Proof. We can assume $\Phi$ is irreducible. Also, Lemmal7.1(a),(b) may be applied with $\lambda=0$. According to (b), the conclusion of (a) holds, unless $\operatorname{dim} H^{1}(G, L(\mu))=1$. We may thus assume, without loss, that $j=0$ and that $\mu_{0} \neq 0$. In particular, $H^{0}\left(G_{1}, L\left(\mu_{0}\right)\right)=0$, and $H^{1}(G, L(\mu))$ injects into $H^{1}\left(G_{1}, L(\mu)\right)$; in fact, $H^{1}(G, L(\mu)) \cong H^{1}\left(G_{1}, L(\mu)\right)^{G / G_{1}}$. Write $\mu=\mu_{0}+p \mu_{1}, \mu_{1} \in X^{+}$. Then

$$
H^{1}\left(G_{1}, L(\mu)\right)^{G / G_{1}} \cong \operatorname{Hom}_{G}\left(L\left(\mu_{1}\right)^{\star}, H^{1}\left(G_{1}, L\left(\mu_{0}\right)\right)^{(-1)}\right),
$$

where $L\left(\mu_{1}\right)^{\star}$ is the module dual to $L\left(\mu_{1}\right)$. Hence, $\operatorname{dim} H^{1}(G, L(\mu))$ is bounded by the number of $G$-composition factors in $H^{1}\left(G_{1}, L\left(\mu_{0}\right)\right)$.

Since there are only a finite number of possible restricted weights $\mu_{0}$, we have proved there exists a constant $C(\Phi, p)$, depending on both $\Phi$ and the prime $p$, such that if $\mu \in X^{+}$, then $\operatorname{dim} H^{1}(G, L(\mu)) \leq C(\Phi, p)$, when $G$ is the semisimple, simply connected group over $k=\overline{\mathbb{F}}_{p}$ having root system $\Phi$.

Next, we prove the second part of the theorem with $p>h$. For each root system $\Phi$, there exists a constant $D(\Phi)$ such that if $p>D(\Phi)$, the LCF holds for all regular $\lambda \in X_{1}^{+}$; see [4]. We will also choose $D(\Phi) \geq h$. Assume that $p>D(\Phi)$. Let $\mu \in X^{+}$be so that $\mu_{0} \neq 0$ and $H^{1}(G, L(\mu)) \neq 0$. By Theorem 7.2 .

$$
1 \leq \operatorname{dim} H^{1}(G, L(\mu)) \leq \operatorname{dim} \operatorname{Ext}_{\mathcal{C}_{\zeta}}^{1}\left(L_{\zeta}(0), L_{\zeta}(\mu)\right) .
$$

By the discussion preceding the theorem, which makes use of Lemma 7.6 proved below, the right-hand side is bounded by $E_{0}(\Phi)$. This proves the theorem for $p>h$.

Finally, putting things together, we see that $C(\Phi):=\max \left\{E_{0}(\Phi), C(p, \Phi), p \leq\right.$ $D(\Phi)\}$ satisfies the requirements of the theorem.

We will now apply these results to generic cohomology for the infinite families of finite groups of Lie type. Let $q=p^{d}$. These groups fall into several classes: (1) the split groups $A_{n}(q), B_{n}(q), \ldots, E_{8}(q) ;(2)$ the (Steinberg) twisted groups ${ }^{2} A_{n}(q), \ldots,{ }^{2} E_{6}(q)$; (3) the Suzuki groups ${ }^{2} B_{2}\left(2^{2 n+1}\right)$; and (4) the Ree groups ${ }^{2} F_{4}\left(2^{2 n+1}\right)$ and ${ }^{2} G_{2}\left(3^{2 n+1}\right) 10$ The reader is referred to [19, Ch. 3]. It will be convenient, however, to denote these groups as ${ }^{i} G(q)$. For example, if $G=S L_{n}(k)$, then ${ }^{2} G(q)$ denotes ${ }^{2} A_{n-1}(q) 11$

Fix $G$ and an infinite family $\left\{{ }^{i} G(q)\right\}$. Here $i$ is fixed and $q$ is allowed to vary over appropriate powers of $p$, as indicated above. For any finite-dimensional rational $G$ module $V$ and positive integer $n$, the generic cohomology of $V$ in degree $n$ is defined to be the common limit

$$
H_{\text {gen }}^{n}(G, V):=\lim _{q \rightarrow \infty} H^{n}\left({ }^{i} G(q), V\right)=\lim _{m \rightarrow \infty} H^{n}\left(G, V^{(m)}\right) .
$$

In [18, 21] it is shown that this limit is achieved for the split $i=1$ cases for $q$ or $m$ sufficiently large. (The characteristic $p$ is fixed in [18, but allowed to vary in [21].) Paper [7] treats these results in the remaining cases $i>112$ Applying Theorem 7.3 to (7.3.2), we obtain the following result. It will be improved in Theorem 7.10.

\footnotetext{
${ }^{10}$ Although we carry through our method for the Ree and Suzuki groups, we could also just quote the work of P. Sin [42, 43], and [43]; see the comments following Theorem 7.10.

${ }^{11} \mathrm{We}$ are using the notation of [19]. Some authors would denote this group by ${ }^{2} A_{n-1}\left(q^{2}\right)$.

${ }^{12}$ In 14 it is shown (in general) that, for any $m, H^{n}\left(G, V^{(m)}\right) \hookrightarrow H_{\text {gen }}^{n}(G, V)$, so that $H_{\text {gen }}^{n}(G, V)$ is the (finite) directed union of the cohomology of the twisted modules $V^{(m)}$.
} 
Theorem 7.4. The number $\operatorname{dim} H_{\text {gen }}^{1}(G, L)$ is, for all irreducible rational $G$-modules $L$, bounded by a constant depending only on $\Phi$, and not on $p$ and $L$.

Remarks 7.5. (a) In [23], Guralnick conjectured that there exists a constant $C$ such that if $G$ is a finite group acting faithfully on an absolutely irreducible module $L$, then $\operatorname{dim} H^{1}(G, L) \leq C$. In [24], it is suggested that, in fact, $\operatorname{dim} H^{1}(G, L) \leq 2$ in all cases. Although this specific guess is now known to be false, the original conjecture on a universal bound remains open; cf. [39, 25], and [10]. Both Guralnick and the authors of this paper believe, even if it is found that there is no universal bound, it should be the case that the dimension grows very slowly. Theorem 7.4 provides positive evidence for this philosophy and is even consistent with the original conjecture (which Guralnick reconfirmed at the June 2007 AIM workshop).

(b) This paper considers the cohomology of the finite groups of Lie type with coefficients in a nontrivial irreducible module $L$ in the defining characteristic $p$ of the ambient algebraic group $G$. The generic cohomology is only partly developed in the cross-characteristic case where the module $L$ is taken over a field of characteristic different from $p$. There is a reasonably satisfactory theory in type $A[16$. Considerably more is known about cross-characteristic cohomology with trivial coefficients [38] for $G L_{n}(q)$.

The following lemma completes the proof of Theorem 7.3 and is needed for the proof of Theorem 7.7. We conjecture that the assumption of regularity on $\lambda$ can be removed. Of course, in the quantum case, one need not require that $p$ be a prime, but we assume this to avoid the discussion of even more special cases.

Lemma 7.6. There is a constant $\widehat{C}(\Phi)$ depending only on the root system $\Phi$ with the following property. For any prime $p>2$, and $>3$ if $\Phi$ has a component of type $G_{2}$, let $U_{\zeta}$ be defined for the root system $\Phi$ and the pth root of unity $\zeta$. Then $\operatorname{dim} \operatorname{Ext}_{\mathcal{C}_{\zeta}}^{1}\left(L_{\zeta}(\lambda), L_{\zeta}(\mu)\right) \leq \widehat{C}(\Phi), \forall \lambda \in X_{\text {reg }}^{+}, \mu \in X^{+}$.

Proof. Since $\lambda \in X_{\mathrm{reg}}^{+}, \mu \in X_{\mathrm{reg}}^{+}$by the linkage principle. By the translation principle, we can assume $\lambda, \mu \in W_{p} \cdot(-2 \rho)$. As noted above Theorem [7.3]

$$
\operatorname{dim} \operatorname{Ext}_{\mathcal{C}_{\zeta}}^{1}\left(L_{\zeta}(y \cdot(-2 \rho)), L_{\zeta}(w \cdot(-2 \rho))\right)=\mu(y, w)
$$

is independent of $p$, if $p>h$. The conditions discussed on $y, w$ (immediately following (7.2.1) ) are equivalent to the assertions that $y \cdot(-2 \rho), w \cdot(-2 \rho) \in X^{+}$, so that (7.6.1) applies to all groups $\operatorname{Ext}_{\mathcal{C}_{\zeta}}^{1}\left(L_{\zeta}(\lambda), L_{\zeta}(\mu)\right)$. Thus, it suffices simply to bound $\operatorname{dim} \operatorname{Ext}_{\mathcal{C}_{\zeta}}^{1}\left(L_{\zeta}(\lambda), L_{\zeta}(\mu)\right)$ for some prime $p>h$ and $p=h$ if $h$ is prime. Such a bound is easy to find for each prime $p$, without even requiring regularity of $\lambda$ : Without loss, we may assume $\lambda \leq \mu$. Let $\mathrm{St}_{\zeta} \in \mathcal{C}_{\zeta}$ denote the irreducible (quantum Steinberg) module with high weight $(p-1) \rho$. Then $\mathrm{St}_{\zeta}$ is well known to be a (self-dual) projective module, as is $\mathrm{St}_{\zeta} \otimes \mathrm{St}_{\zeta} \otimes L_{\zeta}(\lambda)$. Consequently, $\operatorname{dim} \operatorname{Ext}_{\mathcal{C}_{\zeta}}^{1}\left(L_{\zeta}(\lambda), L_{\zeta}(\mu)\right)$ is at most the dimension of the $\mu$-weight space in $\mathrm{St}_{\zeta} \otimes \mathrm{St}_{\zeta} \otimes L_{\zeta}(\lambda)$.

If $\nu$ is a weight of $\mathrm{St}_{\zeta} \otimes \mathrm{St}_{\zeta}$, the dimension of the $\mu-\nu$ weight space of $L_{\zeta}(\lambda)$ is at most the number of ways of writing $\lambda-(\mu-\nu) \leq \nu \leq(2(p-1) \rho$ as a sum of positive roots. Also, $\operatorname{dim} \mathrm{St}_{\zeta} \otimes \mathrm{St}_{\zeta}=p^{|\Phi|}$. Thus, if $\mathfrak{p}$ is the Kostant partition function, $\widehat{C}(\Phi, p)=p^{|\Phi|} \mathfrak{p}(2(p-1) \rho)$ bounds $\operatorname{dim} \operatorname{Ext}_{\mathcal{C}_{\zeta}}^{1}\left(L_{\zeta}(\lambda), L_{\zeta}(\mu)\right)$. This completes the proof of the lemma. 
Theorem 7.7. There is a constant $\widehat{C}=\widehat{C}(\Phi)$ depending only on the root system $\Phi$ with the following property: If $\lambda \in X^{+}$with $\lambda_{j}$ regular for each index $j \geq 0$, then $\operatorname{dim} \operatorname{Ext}_{G}^{1}(L(\lambda), L(\mu)) \leq \widehat{C} \forall \mu \in X^{+}$. If $p>h$ is such that the $L C F$ holds for all regular weights in $X_{1}^{+}$, we may take $\widehat{C}=E(\Phi)$ (defined in terms of Kazhdan-Lusztig polynomials in (7.2.1)).

Proof. Necessarily, $p \geq h$, since the hypothesis states that regular weights exist. If $p=2$, then $h=2$ and all irreducible components of $\Phi$ are of type $A_{1}$. In this case, all weight spaces in all Weyl modules $\Delta(\lambda)$ have dimensions $\leq 1$. In general, if $\lambda \leq \mu, \operatorname{dim} \operatorname{Ext}_{G}^{1}(L(\lambda), L(\mu))$ is at most the multiplicity of the module $L(\lambda)$ as a composition factor of $\nabla(\mu)$ or, equivalently, of $\Delta(\mu)$. So, the theorem certainly holds in this case.

If $p \neq 2$, part (a) of Lemma 7.1 applies to $\operatorname{Ext}_{G}^{1}(L(\lambda), L(\mu))$. Thus, we may assume $\lambda_{0} \neq \mu_{0}$. Also, applying the Hochschild-Serre sequence, we find that

$$
\operatorname{Ext}_{G}^{1}(L(\lambda), L(\mu)) \stackrel{\sim}{\rightarrow} \operatorname{Ext}_{G_{1}}^{1}(L(\lambda), L(\mu))^{G / G_{1}} .
$$

Let St denote the irreducible and self-dual (Steinberg) module with high weight $(p-1) \rho$. Then $\mathrm{St}$ is projective as a $G_{1}$-module, as is any module $\operatorname{St} \otimes V$, for $V \in G_{1^{-}}$ mod. The $G$-module $S:=\mathrm{St} \otimes \mathrm{St} \otimes L(\lambda)$ is $G_{1}$-projective. Let $N$ be the kernel of the natural map $\eta: S \rightarrow k \otimes L(\lambda) \cong L(\lambda)$ (defined by the self-duality of St), and form the short exact sequence $0 \rightarrow N \rightarrow S \stackrel{\eta}{\rightarrow} L(\lambda) \rightarrow 0$ of $G$-modules. If $M$ is any $G_{1}$-module, let $r(M)$ denote the $G_{1}$-radical of $M$, the smallest $G_{1}$ submodule of $M$ whose corresponding factor module is completely reducible. Of course, if $M$ is a $G$-module, then so is $r(M)$. We claim that the natural sequence of restriction maps

$$
\operatorname{Hom}_{G_{1}}(S, L(\mu)) \stackrel{\alpha}{\rightarrow} \operatorname{Hom}_{G_{1}}(N, L(\mu)) \stackrel{\beta}{\rightarrow} \operatorname{Hom}_{G_{1}}(r(S) \cap N, L(\mu)) \rightarrow 0
$$

is an exact sequence.

First, any $G_{1}$-module map $S \rightarrow L(\mu)$ has $r(S)$ in its kernel, so that $\beta \circ \alpha=0$. Next, $r(N) \subseteq r(S)$, so that $r(S) \cap N$ is a direct summand of $N / r(N)$ as a $G_{1}$ module. Thus, $\beta$ is surjective. Also, $N /(r(S) \cap N) \cong(N+r(S)) / r(S)$ is naturally isomorphic to a direct summand of $S / r(S)$. If $f: N \rightarrow L(\mu)$ is a $G_{1}$-module map vanishing on $r(S) \cap N$, then there is a map $g: S / r(S) \rightarrow L(\mu)$ whose restriction to $(N+r(S)) / r(S)$ is the same as the map induced by $f$. Inflating $g$ to $S$, we find that the restriction of $g$ to $N$ is $f$. Thus, the kernel of $\alpha$ is contained in the image of $\beta$, and (7.7.2) is exact, proving the claim.

The standard "projective resolution" definition of $\operatorname{Ext}_{G_{1}}^{1}$ gives a $G$-module isomorphism $\operatorname{Hom}_{G_{1}}(r(S) \cap N, L(\mu)) \cong \operatorname{Ext}_{G_{1}}^{1}(L(\lambda), L(\mu))$. Taking $G$-fixed points and using (7.7.1), $\operatorname{Hom}_{G}(r(S) \cap N, L(\mu)) \cong \operatorname{Ext}_{G}^{1}(L(\lambda), L(\mu))$, so dim $\operatorname{Ext}_{G}^{1}(L(\lambda), L(\mu))$ is at most the dimension of the $\mu$-weight space of $S$, just as in the argument for Lemma 7.6. Continuing as in that argument, taking $\lambda \leq \mu$, we find that $p^{|\Phi|} \mathfrak{p}(2(p-1) \rho)$ bounds $\operatorname{dim} \operatorname{Ext}_{G}^{1}(L(\lambda), L(\mu))=\operatorname{dim}_{\operatorname{Ext}_{G}}^{1}(L(\mu), L(\lambda))$. Thus, the theorem holds for individual primes. The remaining details, for $p>h$ large enough so that the $L C F$ holds for restricted weights, parallel the proof of Theorem 7.3 , making use of Lemma 7.6. We leave these details to the reader.

Remarks 7.8. (a) In Theorem 7.7, we conjecture that the assumption that the $\lambda_{j}$ be regular is not needed, at least for $j>0$. 
(b) We do not know if there are similar results to Theorem 7.7 or to Theorem 7.3 for higher degree $\mathrm{Ext}_{G^{-}}$-groups or cohomology (in a fixed degree). The quantum analogue seems very likely to hold, at least for regular weights, because the Ext $_{A}{ }_{A}$ algebra of a Koszul algebra $A$ is generated by its terms in degree one.

(c) In [1, Theorem 5.6], Andersen gives a nice reduction, if $p \geq 3 h-3$, of the calculation of $\operatorname{Ext}_{G}^{1}(L(\lambda), L(\mu))$ to a calculation of tensor products and certain other $\operatorname{Ext}_{G}^{1}$-groups. In our notation, his formula is

$$
\begin{aligned}
& \operatorname{Ext}_{G}^{1}(L(\lambda), L(\mu)) \\
& \quad=\sum_{\nu} \operatorname{dim} \operatorname{Ext}_{G}^{1}\left(L\left(\lambda_{j}+p \nu\right), L\left(\mu_{j}\right)\right) \operatorname{dim} \operatorname{Hom}_{G}\left(L\left(\lambda^{(j+1)}\right), L(\nu) \otimes L\left(\mu^{(j+1)}\right)\right),
\end{aligned}
$$

where, as in Theorem 7.2, $j$ is the smallest index $i$ with $\lambda_{i} \neq \mu_{i}$. The weight $\nu$ ranges over dominant weights required to satisfy the inequality

$$
\lambda_{j}+p \nu \leq 2(p-1) \rho+w_{0}\left(\mu_{j}\right) .
$$

These weights $\nu$ are quite small (though [1, Remark 5.7(i)] incorrectly, but inconsequentially, overstates the case for this - $\nu$ need not be a sum of distinct fundamental weights). For such a $\nu, \operatorname{dim} \operatorname{Ext}_{G}^{1}\left(L\left(\lambda_{j}+p \nu\right), L\left(\mu_{j}\right)\right)$ may be calculated in terms of Kazhdan-Lusztig polynomials, provided $\lambda_{j}$ is regular (again, [1, Remark 5.7(iv)] is too optimistic, and the assumption of regularity is still needed, though it may, one day, be removed). At the time of [1] quantum groups did not exist, but it seems likely that the same methods should yield a similar formula, with even weaker assumptions, to (7.8.1) in the quantum case, if $j=0$. The right-hand Hom term in (7.8.1) would then be taken over the enveloping algebra $U\left(\mathfrak{g}_{\mathbb{C}}\right)$. Comparison of the Hom terms in (7.8.1) would provide an alternate proof of Theorem 7.2 without the use of Theorem 6.8, though it would require the additional assumption $p \geq 3 h-3$. Even without such a quantum version, one may use (7.8.1) and the weight space philosophy of [1, Remark $5.7(\mathrm{v})$ ] to obtain an alternate proof of some version of Theorems 7.3 and 7.7, albeit without the same bounds, and with the additional assumption $p \geq 3 h-3$.

The following consequence of Theorem 7.7 is stated without further proof.

Theorem 7.9. There is a constant $\widehat{C}=\widehat{C}(\Phi)$, depending only on $\Phi$, such that

$$
\operatorname{dim} \operatorname{Ext}_{G, \text { gen }}^{1}(L(\lambda), L(\mu)):=H_{\text {gen }}^{1}\left(G, \operatorname{Hom}_{k}(L(\lambda), L(\mu)) \leq \widehat{C}\right.
$$

for $\lambda, \mu \in X^{+}$such that $\lambda_{j}$ is regular for each $j$. If $p>h$ is such that the $L C F$ holds for all regular weights in $X_{1}^{+}$, we may take $\widehat{C}=E(\Phi)$.

To obtain a result similar to Theorem 7.4 for the cohomology of the actual finite groups of Lie type, as opposed to the generic cohomology, we can apply Theorem 7.7 and some results of C. Bendel, D. Nakano, and C. Pillen. We will also use work of P. Sin for some special cases. Fix a root system $\Phi$. Fix a set $\Pi$ of simple roots for $\Phi$ and let $\tau$ be a graph automorphism of $\Phi$ (stabilizing $\Pi$ ). For weight $\xi$, put $\widehat{\xi}:=\tau(\xi)^{\star}=-w_{0} \tau(\xi)$ (the image of $\tau(\xi)$ under the opposition involution $(-)^{\star}$ ). For any semisimple, simply connected group $G$ over an algebraically closed field $k$ of characteristic $p>0$ with root system $\Phi$, we can regard $\tau$ as an automorphism of $G$ commuting with the standard Frobenius morphism $F: G \rightarrow G$ (which defines 
the split form of $G$ ). Let $\sigma:=F^{r} \circ \tau$. By [ $[$, Thm. 5.1], there is a finite-dimensional, rational $G$-module $\mathcal{G}_{r}(k)$ such that, for $\lambda, \mu \in X_{r}^{+}$,

$$
\operatorname{Ext}_{G_{\sigma}}^{1}(L(\lambda), L(\mu)) \cong \operatorname{Ext}_{G}^{1}\left(L(\lambda), L(\mu) \otimes \mathcal{G}_{r}(k)\right)
$$

In the following theorem, recall $G$ is a connected, simply connected, semisimple algebraic group with root system $\Phi$ over an algebraically closed field $k$ of characteristic $p>0$. The theorem applies to the finite groups $G_{\sigma}$ of Lie type in this case. Finite groups of Lie type can be considered without this assumption on $G$ (e.g., $\left.G_{\sigma}=P G L_{n}(q)\right)$, but this case is the central one of interest. In applying the results of [ㅁ, we will assume that $\sigma$ has the form of the previous paragraph, so that $G_{\sigma}$ is not a Ree or Suzuki group. While it seems likely that the methods of [8] can be applied in these cases, it will be more efficient just to quote some results of Sin in the Ree and Suzuki cases.

Theorem 7.10. There is a constant $C^{\prime}=C(\Phi)^{\prime}$, depending only on $\Phi$, with the following property: Let $\sigma: G \rightarrow G$ be an endomorphism such that $G_{\sigma}$ is a finite group. Then $\operatorname{dim} H^{1}\left(G_{\sigma}, L\right) \leq C^{\prime}$ for all irreducible $k G_{\sigma}$-modules $L$.

Proof. First, assume that $G_{\sigma}$ is not of Ree or Suzuki type. Then we can assume that $\sigma=F^{r} \circ \tau=\tau \circ F^{r}$ for some positive integer $r$ and a graph automorphism $\tau$ of $G$.

Assume that $p>3(h-1)$. Let $\Gamma$ be the set of dominant weights $\lambda$ which satisfy $\left(\lambda, \alpha_{0}^{\vee}\right)<2(h-1)$. Below we will use the fact that if $p>3(h-1)$, then any $\lambda \in \Gamma$ is a regular dominant weight in the bottom $p$-alcove. Also, if $p^{r-1} p>4 h-6$, [8, Thm. 4.7] proves that $\mathcal{G}_{r}(k)$ has a filtration with sections $\nabla(\hat{\lambda}) \otimes \nabla(\lambda)^{(r)}$ for $\lambda \in \Gamma$. Further, such a section occurs exactly once. Since $p>3(h-1)$, each $\lambda \in \Gamma$ is a regular weight in the bottom $p$-alcove. Also, $\lambda \in \Gamma$ implies that $\hat{\lambda} \in \Gamma$ as well. Thus, $\nabla(\hat{\lambda}) \cong L(\widehat{\lambda})$ and $\nabla(\lambda) \cong L(\lambda)$ for $\lambda \in \Gamma$. In the $p$-adic expansion $\sum_{j>0} p^{j} \lambda_{j}$ of $\hat{\lambda}+p^{r} \lambda$, we have $\lambda_{0}=\hat{\lambda}, \lambda_{r}=\lambda$, and $\lambda_{i}=0$ otherwise. Thus, each $\lambda_{j}^{\star}$ is a regular weight, so Theorem 7.7 implies there is a constant bounding all dimensions $\operatorname{dim} \operatorname{Ext}_{G}^{1}\left(L\left(\widehat{\lambda}+p^{r} \lambda\right)^{*}, L(\mu)\right)=\operatorname{dim} \operatorname{Ext}_{G}^{1}\left(L\left(\widehat{\lambda}^{\star}+p^{r} \lambda^{\star}\right), L(\mu)\right)$ for all $\mu \in X^{+}$. Since $\Gamma$ is a finite set, independent of $r, p$, it follows from (7.9.1) that $H^{1}\left(G_{\sigma}, L\right)$ is bounded by a constant depending only on the root system. (In fact, the conditions imposed on $q=p^{r}$ hold with a finite number of exceptions.) Finally, if $p \leq 3(h-1)$, for all but a finite number of cases, we have $r \geq 2$ and $p^{s-1}(p-1)>h$, where $s$ is the largest integer $\leq r / 2$. Then [8, Thm. 5.5] implies that, given any irreducible $G_{\sigma}$-module $L$, there is an irreducible, rational $G$-module $L^{\prime}$ such that $H^{1}\left(G_{\sigma}, L\right) \cong H^{1}\left(G, L^{\prime}\right)$. Now we can apply Theorem 7.3 .

This completes the proof unless $G_{\sigma}$ is of Ree or Suzuki type. In these cases, work of $\operatorname{Sin}$ [42, 43, and 44] bounds $\operatorname{dim} H^{1}\left(G_{\sigma}, L\right), L$ irreducible (and also bounds $\operatorname{dim} \operatorname{Ext}^{1}\left(L, L^{\prime}\right), L, L^{\prime}$ irreducible, in some cases). In fact, in these papers, explicit calculations of dimensions are given. The integer 2 is an upper bound on $\operatorname{dim} H^{1}\left(G_{\sigma}, L\right)$ in all the Ree and Suzuki cases. This completes the proof.

We conclude this paper with the following result and a remark. (See especially the last part of Remark 7.12(c), which is relevant to the theorem.) 
Theorem 7.11. Assume that $p>h$. Let $\lambda=\tau+p \nu \in X^{+}$, with $0 \neq \tau \in X_{1}^{+}$and $\nu \in X^{+}$. Suppose that $\operatorname{dim} H^{1}(G, L(\lambda))>1$. Then $\tau>p \nu^{\star}$, where $\nu^{\star}:=-w_{0}(\nu) \in$ $X^{+}$.

Proof. By Theorem 7.2, the hypotheses imply that

$$
\operatorname{dim} H^{1}\left(U_{\zeta}, L_{\zeta}(\lambda)\right)=\operatorname{dim}_{\operatorname{Ext}_{\mathcal{C}_{\zeta}}^{1}}\left(L_{\zeta}\left(p \nu^{\star}\right), L_{\zeta}(\tau)\right)>1
$$

By Corollary 5.2 and (1.5.1),

$$
\operatorname{dim} \operatorname{Ext}_{\mathcal{C}_{\zeta}}^{1}\left(L_{\zeta}\left(p \nu^{\star}\right), \nabla_{\zeta}(\tau)\right)=\operatorname{dim}_{\operatorname{Ext}_{G}^{1}}\left(\Delta\left(\nu^{\star}\right)^{(1)}, \nabla(\tau)\right) \leq 1 .
$$

Therefore, if we set $Q_{\zeta}(\tau):=\nabla_{\zeta}(\tau) / L_{\zeta}(\tau)$, the long exact sequence of cohomology forces the conclusion that $\operatorname{Hom}_{\mathcal{C}_{\zeta}}\left(L_{\zeta}\left(p \nu^{\star}\right), Q_{\zeta}(\tau)\right) \neq 0$. Hence, $p \nu^{\star}<\tau$.

Remarks 7.12. (a) Assume that $p>h$ and the LCF holds for all regular weights in $X_{1}^{+}$. Using Lemma 7.1 and Theorem 7.11, we see that, if $\operatorname{dim} H^{1}(G, L(\lambda))>1$, then $\lambda=p^{r} \sigma$, with $\sigma=\sigma_{0}+p \sigma_{1}$, with $0 \neq \sigma_{0} \in X_{1}^{+}$and $\sigma_{1} \in X^{+}$, where $p \sigma_{1}^{\star}<\sigma_{0}$. In addition, $p \sigma_{1}^{\star}$ must be $W_{p}$-linked to $\sigma_{0}$.

(b) Suppose $G=S L_{6}$ for a field $k$ of characteristic $p>h=6$ sufficiently large that the LCF holds for all regular weights in $X_{1}^{+}$. There is a natural isomorphism $W_{p} \rightarrow W_{7}, w \mapsto w^{\prime}$, of Coxeter groups, taking the simple reflections for $W_{p}$ to their exact analogues in $W_{7}$. Choose $w \in W_{p}$ so that $w^{\prime} \cdot(-2 \rho)=4 \varpi_{1}+5 \varpi_{2}+4 \varpi_{3}+$ $5 \varpi_{4}+4 \varpi_{5}$. Let $\lambda=w \cdot(-2 \rho)$. By a calculation of C. McDowell reported in 39, Prop. 3, Cor. 4], $\mu_{w_{0}, w}=3$. Thus, $\operatorname{dim} H^{1}\left(S L_{6}\left(p^{d}\right), L(\lambda)\right) \geq 3$ for $d$ sufficiently large.

(c) The bounds given by Theorem 7.7 (or Theorem 7.9) are roughly exponential in terms of the rank of the root system (and so not very good!). For example, the proof of Lemma 7.6 shows that

$$
\widehat{C}(\Phi, p) \leq p^{|\Phi|} \mathfrak{p}(2(p-1) \rho),
$$

for any prime. Actually, the same statement holds with a similar proof replacing $p$ by $h+1$. Then one can easily bound $\mathfrak{p}(2(\rho-1) \rho)$ by $N ! P(N(h-1))$, where $N=r h / 2$ is the number of positive roots (and $r$ is the rank of $G$ ), and $P$ is the usual partition function. One can also argue with the recursive definition of Kazhdan-Lusztig polynomials to get a bound

$$
E(\Phi) \leq 2^{2|W|},
$$

where $W=W(\Phi)$ is the Weyl group. Presumably none of these estimates is even close. N. Xi 48 has provided elegant theoretical arguments which show the relevant Kazhdan-Lusztig polynomial coefficients for ordinary Weyl groups are often 0 or 1 for good reason. His result implies that Ext ${ }^{1}$-groups for the BGG category $\mathcal{O}$ (for a complex semisimple Lie algebra) are often of dimension $\leq 1$, at least in type $A$. This phenomenon is very similar to what Guralnick (and, subsequently, many others) have empirically observed for finite groups. It is similar in spirit to Theorem 7.11.

Notes added in proof.

1. The third author wishes here to withdraw the claim of [39, §4] that there are examples in cross-characteristic of faithful absolutely irreducible modules $L$ for finite groups $G=G(q)$ of Lie type with $\operatorname{dim} H^{1}(G, L) \geq 3$. The corresponding 
claim in defining characteristic proved in the previous sections of 39] is unaffected (and gives infinitely many examples). The problem is that the role played by the trivial module in the defining characteristic case unfortunately is played in the cross-characteristic cases discussed in [39, §4] by a non-trivial module, replacing $H^{1}$ by an $\mathrm{Ext}^{1}$.

2. In connection with Remark 6.7, we point out that in his paper "Darstellungen halbeinfacher Gruppen und ihrer Frobenius-Kerne" (J. Reine und Angew. Math. 317 (1980), 157-199), Jantzen shows that Conjecture 6.6 holds generically (i.e., for dominant weights far enough from the walls of the dominant chamber) for any group

$G$; see $\S 3.4$ and Satz 3.8. On the other hand, in $\S 3.13$, he verifies the conjecture for all dominant weights in type $A_{2}$. (Thus, Conjecture 6.5 also holds in type $A_{2}$.)

\section{REFERENCES}

[1] H. Andersen, Extensions of modules for algebraic groups, Amer. J. Math. 106 (1984), 489504. MR737781 (86g:20056)

[2] H. Andersen, p-filtrations and the Steinberg module, J. Algebra 244 (2001), 664-683. MR:1859043 (2002k:20080)

[3] H. Andersen and J. Jantzen, Cohomology of induced representations of algebraic groups, Math. Ann. 269 (1984), 487-525. MR766011 (86g:20057)

[4] H. Andersen, J. Jantzen, and W. Soergel, Representations of quantum groups at a pth root of unity and of semisimple groups in characteristic p, Astérique 220 (1994).

[5] H. Andersen, P. Polo, and K. Wen, Representations of quantum algebras, Invent. Math. 104 (1991), 571-604. MR1094046 (92e:17011)

[6] M. Aschbacher and L. Scott, Maximal subgroups of finite groups J. Algebra 192 (1985), 44-80. MR772471 (86m:20029)

[7] G. Avrunin, Generic cohomology for twisted groups, Trans. Amer. Math. Soc. 268 (1981), 247-253. MR628457 (82m:20048)

[8] C. Bendel, D. Nakano, and C. Pillen, Extensions of finite groups of Lie type II: filtering the truncated induction functor, Contemporary Math. 413 (2006), 1-23. MR 2262362 (2007i:20074)

[9] N. Bourbaki, Groupes et algèbres de Lie, IV, V, VI, Hermann (1968). MR0240238 (39:1590)

[10] J. Bray and R. Wilson, Examples of 3-dimensional 1-cohomology for absolutely irreducible modules of finite simple groups, to appear.

[11] C. Chevalley, Sur certains groupes simples, Töhoku Math. J. 7 (1955), 14-66. MR0073602 $(17: 457 \mathrm{c})$

[12] E. Cline, B. Parshall, and L. Scott, Detecting rational cohomology of algebraic groups, J. London Math. Soc. 28 (1983), 293-300. MR713384 (84j:20046)

[13] E. Cline, B. Parshall, and L. Scott, Abstract Kazhdan-Lusztig theories, Tôhoku Math. J. 45 (1993), 511-534. MR1245719 (94k:20079)

[14] E. Cline, B. Parshall, and L. Scott, The homological dual of a highest weight category, Proc. London Math. Soc. 68 (1994), 294-316. MR1253506 (94m:20093)

[15] E. Cline, B. Parshall, and L. Scott, Simulating perverse sheaves in modular representation theory, Proc. of Symposia in Pure Math. 56(1) (1994), 63-104. MR1278701 (95i:20061)

[16] E. Cline, B. Parshall, and L. Scott, Rational and $q$-generic representation theory, Publ. RIMS (Kyoto) 35 (1999), 31-90. MR1681236 (2000i:20021)

[17] E. Cline, B. Parshall, and L. Scott, An Ext-transfer theorem for algebraic groups, J. Transformation Groups 9 (2004), 213-236. MR2079133 (2005e:20069)

[18] E. Cline, B. Parshall, L. Scott and W. van der Kallen, Rational and generic cohomology, Invent. Math. 39 (1977), 143-163. MR0439856 (55:12737)

[19] J. Conway, R. Curtis, S. Norton, R. Parker, and R. Wilson, Atlas of Finite Groups, Clarendon Press, Oxford (1985). MR827219 (88g:20025)

[20] J. Du and L. Scott, Lusztig conjectures, old and new, I, J. Reine Angew. Math. 455 (1994), 141-182. MR1293877 (95i:20062) 
[21] E. Friedlander and B. Parshall, Cohomology of infinitesimal and discrete groups, Math. Annalen 273 (1986), 353-374. MR824427 (87e:22026)

[22] D. Happel, Triangulated Categories in the Representation of Finite Dimensional Algebras (London Mathematical Society Lecture Note Series) 119 (1988). MR.935124 (89e:16035)

[23] R. Guralnick, The dimension of the first cohomology groups, in: Representation Theory, II, Ottawa (1984), in: Lectures Notes in Math. 1178, Springer (1986), 94-97. MR842479 (87h:20095)

[24] R. Guralnick and C. Hoffman, The first cohomology group and generation of simple groups, in: Groups and Geometries, Siena (1996), in: Trends Math. Birkhäuser (1998), 81-89. MR:1644977 (99h:20087)

[25] R. Guralnick, Cohomology and permutations, Oberwolfach Report No. 15/2006 (2006), 1315. (This article reports on the preprints, "Presentations of finite simple groups: cohomological and profinite approaches" and "Presentations of finite simple groups: a quantative approach" by R. Guralnick, W. Kantor, M. Kassabov, and A. Lubotzky.)

[26] J. C. Jantzen, Representations of algebraic groups, 2nd ed., American Mathematical Society (2003). MR2015057 (2004h:20061)

[27] J. C Jantzen, Lectures on Quantum Groups, Grad. Studies in Math. 6, Amer. Math. Soc., 1996. MR1359532 (96m:17029)

[28] S. I. Kato, On Kazhdan-Lusztig polynomials for affine Weyl groups, Adv. Math. 55 (1985), 103-130. MR772611 (86d:20048)

[29] D. Kazhdan and G. Lusztig, Tensor structures arising from affine Lie algebras, I-III; IV-VI, J. Amer. Math. Soc. 6 (1993), 905-1011; 7 (1994), 335-453.

[30] S. Kumar, N. Lauritzen, and J. Thomsen, Frobenius splitting of cotangent bundles of flag varieties, Invent. Math. 136 (1999), 603-621. MR1695207 (2000g:20088)

[31] Z. Lin, Highest weight modules for algebraic groups arising from quantum groups, J. Algebra 208 (1998), 276-303. MR.1644011 (99k:20087)

[32] G. Lusztig, Some problems in the representation theory of finite Chevalley groups, Proc. Symp. Pure Math. 37 (1980), 313-317. MR604598 (82i:20014)

[33] G. Lusztig, Quantum groups at roots of 1, Geom. Dedicata 35 (1990), 89-114. MR1066560 (91j:17018)

[34] B. Parshall and L. Scott, Quantum Weyl reciprocity for cohomology, Proc. London Math. Soc. 90(3) (2005), 655-688. MR2137826 (2006d:20089)

[35] B. Parshall and L. Scott, Extensions, Levi subgroups and character formulas, J. Algebra, 319 (2008), no. 2, 680-701. MR2381802 (2008j:20136)

[36] B. Parshall and L. Scott, Beyond the Jantzen region, Oberwolfach Report No. 15/2006 (2006), 26-30.

[37] B. Parshall and L. Scott, Some $\mathbb{Z} / 2$-graded representation theory, The Quarterly J. Math, in press.

[38] D. Quillen, On the cohomology and $K$-theory of the general linear groups over a finite field. Ann. of Math. 96 (1972), 552-586. MR0315016 (47:3565)

[39] L. Scott, Some new examples in 1-cohomology, J. Algebra 260 (2003), 416-425. MR.1976701 (2004f:20092)

[40] L. Scott, Semistandard filtrations and highest weight categories, Michigan J. Math., to appear.

[41] L. Scott and N. Xi, Some non-trivial Kazhdan-Lusztig coefficients of an affine Weyl group of type $\widetilde{A}_{n}$, to appear.

[42] P. Sin, Extensions of simple modules for $G_{1}\left(3^{n}\right)$ and ${ }^{2} G_{2}\left(3^{m}\right)$, Proc. London Math. Soc. 66 (1993), 327-357. MR.1199070 (94a:20020)

[43] P. Sin, Extensions of simple modules for $S p_{4}\left(2^{n}\right)$ and $S u z\left(2^{m}\right)$, Bull. London Math. Soc. 24 (1992), 159-164. MR1148676 (93b:20025)

[44] P. Sin, The cohomology of degree 1 of the group $F_{4}$ in characteristic 2 with coefficients in a simple module J. Algebra 164 (1994), 695-717. MR1272111 (95c:20063)

[45] R. Steinberg, Lectures on Chevalley Groups (notes by J. Faulkner and R. Wilson), Yale University (1968). MR0466335 (57:6215)

[46] T. Tanisaki, Character formulas of Kazhdan-Lusztig type, Representations of finite dimensional algebras and related topics in Lie theory and geometry, Fields Inst. Commun. 40, Amer. Math. Soc., Providence, RI (2004) 261-276. MR2057399 (2005h:17046) 
[47] A. Wiles, Modular elliptic curves and Fermat's last theorem, Annals of Math. 141 (1995), 443-551. MR1333035 (96d:11071)

[48] N. Xi, The leading coefficient of certain Kazhdan-Lusztig polynomials for the permutation group $\mathfrak{S}_{n}$, J. Algebra 285 (2005), 136-145. MR2119108(2006a:20013)

Department of Mathematics, University of Oklahoma, Norman, Oklahoma 73019

E-mail address: ecline@math.ou.edu

Department of Mathematics, University of Virginia, Charlottesville, Virginia 22903

E-mail address: bjp8w@virginia.edu

Department of Mathematics, University of Virginia, Charlottesville, Virginia 22903

E-mail address: 1ls21@virginia.edu 